\title{
Double charge exchange in ion-atom collisions using distorted wave theories with two-electron continuum intermediate states in one or both scattering channels
}

\section{Dževad Belkić ${ }^{1,2}$}

Received: 29 August 2018 / Accepted: 15 October 2018 / Published online: 1 November 2018 (c) The Author(s) 2018

\begin{abstract}
A general quantum-mechanical formalism is reviewed for double electron capture from heliumlike atomic systems by fast nuclei. The development is carried out with and without the distorted wave theory by fulfilling the correct boundary conditions. These refer to the required asymptotic behaviors of the total scattering wave functions and their appropriate connections to the perturbation interactions that produce the transitions from the initial to the final states of the system. In this general formulation any choice is allowed for the pairs of the distorting potentials and the related distorted wave functions as long as the correct boundary conditions are satisfied. This is the case with the four-body versions of several most frequently used methods (continuum distorted wave: CDW-4B, boundary-corrected continuum intermediate state: BCIS-4B, Born distorted wave: BDW-4B, continuum distorted wave initial/final state: CDW-EIS/EFS-4B, and the boundary-corrected first Born: CB1-4B). A comparative analysis of these methods makes in evidence both their similarities and differences. For example, the most illustrative is the juxtaposition of the post BDW-4B and CDW-EIS4B methods. They share the same distorting potential in the exit channel. The only difference is in the coordinates from the Coulomb logarithmic phases in the initial distorted wave functions. This difference is completely negligible in the asymptotic scattering regions. Yet, for e.g. double electron capture from helium by alpha particles, the total cross sections from these two methods differ by 1-3 orders of magnitudes. The BDW-4B method is in agreement with experimental data at high impact energies. In sharp contrast, within its validity domain of impact energies, the CDW-EIS-4B method underestimates the measured data by orders of magnitude. This shows that what matters is not solely the correct asymptotes of distorted wave functions, but rather how they affect the contributions to the integrals over the entire regions in the T-matrix elements for total cross sections. Such insights help understand the assessment of the overall validity and relative performance of various methods, and can provide a versa-
\end{abstract}

Dževad Belkić

Dzevad.Belkic@ki.se

Extended author information available on the last page of the article 
tile guidance for improving the existing approximations for double charge exchange in fast ion-atom collisions.

Keywords High energy atomic collisions · Correct boundary conditions · Double charge exchange $\cdot$ Second-order theories

\section{Contents}

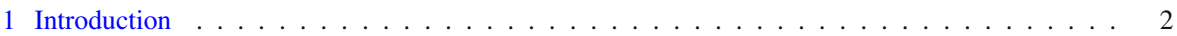

2 General theory with and without the distorted wave formalism . . . . . . . . . . . . . 5

2.1 Coulomb-modified initial and final scattering states without distorted waves . . . . . . . . 5

2.1.1 Eikonal formalism for dominant forward scatterings of heavy particles . . . . . . . . 8

2.1.2 The prior and post forms of the transition amplitudes . . . . . . . . . . . . . . 12

2.2 Coulomb-modified initial and final scattering states with distorted waves . . . . . . . . . . . 14

2.3 Determination of the initial and final distorted waves . . . . . . . . . . . . . . . . 17

2.3 .1 Entrance channel . . . . . . . . . . . . . . . . . . . . . . . . 17

2.3 .2 Exit channel . . . . . . . . . . . . . . . . . . . . . . . 19

2.4 Asymptotic behaviors of distorted waves at large inter-particle distances . . . . . . . . . . 20

2.5 Different choices of the distorting potentials and distorted waves . . . . . . . . . . . . . . 22

2.5.1 Symmetric second-order theories: four-body continuum distorted wave method, CDW-4B 22

2.5.2 Symmetric first-order theories: four-body boundary-corrected first Born method, CB1-4B 28

2.5.3 Asymmetric second-order theories: four-body boundary-corrected continuum intermediate states method, BCIS-4B . . . . . . . . . . . . . . . 32

2.5.4 Asymmetric second-order theories: four-body Born distorted wave method, BDW-4B . 34

2.5.5 Asymmetric second-order theories: four-body continuum distorted wave eikonal initial/final state methods, CDW-EIS/EFS-4B . . . . . . . . . . . . . . . 37

2.5.6 The link between the prior/post BDW-4B and CDW-EFS/EIS-4B methods . . . . . . . 39

2.5.7 The link between the prior/post CDW-4B and CDW-EFS/EIS-4B methods . . . . . . . 41

3 Convergence issues with the Born series for rearrangement collisions . . . . . . . . . . . . . . . 42

4 Illustrations . . . . . . . . . . . . . . . . . . . . . . . . 44

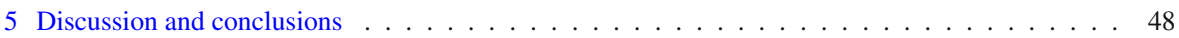

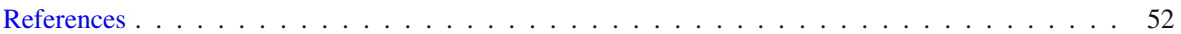

\section{Introduction}

Double charge exchange in ion-atom collisions at intermediate and high energies is prominent among many multi-electron processes [1-4]. These include electron transfer, excitation, ionization their combinations (transfer-excitation, transfer-ionization, ...), etc. Such processes have been studied intensively over the years both theoretically [5-54] and experimentally [55-86]. When there is active participation of two or more electrons from either the projectile or the target or both, we talk about two or multiple electron transfer, excitation, ionization, transfer-excitation, transfer ionization, etc. Stated equivalently, the term multiple electron atomic processes implies that more than one electron has left its initial orbital.

The so-called frozen core approximation has often been invoked in descriptions of such collisional processes. This additional approximation assumes that the electrons that do not participate to the actual transitions (the passive electrons) remain in the final state of the target and/or projectile in the same orbitals which they have occupied in the initial states. Such an approximation is expected to be reasonable at high impact 
energies. Nevertheless, it is pertinent here to emphasize that e.g. a single-electron process, in the strict meaning of the term, cannot occur in collisions involving multielectron atomic systems. The explanation is that an alteration in the orbital energy of one electron (the active electron) would inevitably lead to some changes (albeit perhaps only slight) in the orbital energies of the remaining electrons (meaning that here, in fact, there could be no passive electrons). Such a strictness is often not of a particular concern in many applications that frequently rely upon the frozen core approximation, the notion of passive electrons, the effective or screened nuclear charges, etc.

In particular, for charge-exchange processes, the non-captured electrons are viewed as playing only a static role amounting to merely screening the bare nuclear charge. Various choices of the effective nuclear charges can be made, and this should be done in a consistent manner. Physically, these effective nuclear charges should be close to the nuclear charges that reproduce the orbital energies of the initial and final bound states. One reasonable choice is guided by the fact that the nuclear charge $Z$ and the binding energy $\varepsilon_{n}<0$ of the electron in a hydrogenlike atomic system for the state with the principal quantum number $n$ is $Z=\left(-2 n^{2} \varepsilon_{n}\right)^{1 / 2}$. Similarly, as suggested in Ref. [87], in the case of a multi-electron atomic or ionic target, an electron to be captured from a state of the orbital energy $\varepsilon_{n}^{T}<0$ with the orbital number $n$, the effective nuclear charge $Z_{\mathrm{T}}^{\text {eff }}$ could be chosen to satisfy the hydrogenic-type relationship $Z_{\mathrm{T}}^{\text {eff }}=\left(-2 n^{2} \varepsilon_{n}^{\mathrm{T}}\right)^{1 / 2}$. Here, $\varepsilon_{n}^{\mathrm{T}}$ could be selected as the Roothan-HartreeFock orbital energy for which the tabulated values can be found in Ref. [88] for many multi-electron atomic systems. Also given in Ref. [88] are the variationally determined parameters (expansion coefficients, exponential damping factors) for the analytical forms of the corresponding ground-state wave functions (including some of the excited states) for neutral and ionized atoms. These latter wave functions are linear combinations of the Slater-type orbitals (STO) as the basis set functions.

This type of choice for an effective or screened nuclear charge works quite well in practice [87]. The reason is in the fact that charge-exchange is a very local process. This process occurs with non-negligible probabilities at the places where the initial and/or final bound state wave functions are appreciable. It is at these places that the electrons to be captured experience the screened charge $Z_{\mathrm{T}}^{\text {eff }}$ as an average target nuclear charge. Note that due to their exponential decline with augmentation of distances, the atomic bound state wave functions take on their noticeable values only at small separations between the electrons and their parent nucleus. At high energies, the dominant contribution to charge exchange transition amplitude for complex atomic targets is predominantly determined by the electrons that are closest to their nucleus (the K-shell electrons). Small distances correspond to high momenta. Therefore, even at high impact energies, it is important to use the atomic wave functions whose momentum-space representations accurately describe high momentum components of the electronic states. Momentum-space bound-state wave functions come into play here because the charge exchange transition amplitudes are determined by the overlap integrals of the initial and final scattering states. Such overlap integrals contain the momentum-space bound-state wave functions that are initially given in the coordinate representations. This becomes most obvious from an inspection of the 
well-known transition amplitude in the first-order Oppenheimer-Brinkman-Kramers (OBK) approximation for single electron capture processes [89].

From these remarks one can infer the two main mechanisms, the velocity matching and the Thomas-type double scattering, for charge exchange in the first- and second-order methods, respectively. The first-order methods are based upon the onestep pathways, involving the direct projectile-target interactions alone. Therein, the velocity matching mechanism operates via the circumstance that the dominant contribution to electron capture is due to the near equality between the incident speed and the orbital velocity of the active target electron. High incident velocities require high momentum components from the momentum distribution in the target momentumspace bound-state wave functions. The second-order methods describe the two steps via target ionization followed by capture of the emitted electron. The emitted electron must have high momentum if it is to be captured by a fast projectile. This ionizationcapture mechanism is a quantum-mechanical version of the classical Thomas double scattering. There are two successive elastic rearranging collisions in the Thomas billiard-type twin events. In the first event, the electron is scattered elastically on the projectile through $60^{\circ}$ towards the target nucleus. In the second encounter, the electron scatters again elastically through $60^{\circ}$ on the target nucleus with the velocity $v$ equal to the projectile large speed. This electron is finally captured by the positively charged projectile, since on top of having collinear velocities, the attractive Coulomb potential between these two particles binds them together into a newly formed atomic system.

In the present review, we will focus only upon several selected first- and secondorder methods with the correct boundary conditions for double electron capture from heliumlike targets by heavy nuclei. These are the four-body continuum distorted wave (CDW-4B) [30,31], boundary-corrected continuum intermediate state (BCIS-4B) [32], Born distorted wave (BDW-4B) [41,42], continuum distorted wave initial/final state (CDW-EIS/EFS-4B) [47] and the boundary-corrected first Born (CB1-4B) [33,34] methods. We will illuminate their similarities as well as differences and illustrate their performance in the most frequently studied example of collisions between alpha particles and helium atoms.

Atomic collisions involving multiple electron transitions have been of notable interest over last several decades in a vastly different applications ranging from basic research purposes to technology. These include, but are not limited to:

- Stellar atmospheres, upper atmosphere, inter-stellar medium [90,91].

- Heavy ion accelerators at GSI (Darmstadt), KSU (Kanzas), GANIL (Caen), etc [92]

- Storage ring accelerator such as ESR, CRYRING (at GSI), TSR (Heidelberg), ASTRID (Aarhus), etc [93-96].

- Ion traps (EBIT, Paul trap, Penning trap, ...), ion sources (EBIS, ECRIS, ...), etc [93-95].

- Charge exchange spectroscopy in magnetically confined plasmas [97].

- Hot and dense plasmas $\left(T \geq 10^{6}{ }^{\circ} \mathrm{K}, n_{e} \sim 10^{19} / \mathrm{cm}^{3}-10^{24} / \mathrm{cm}^{3}\right)$, hightemperature thermonuclear fusion by way of inertial confinement accomplished with heavy ion bombardment (at GSI), short high-energy laser pulses (at LMJ: 
Bordeaux, PHOEBUS: Limeil, NOVA, NIS: Livermole, ...) or short intense discharges (Z-pinch), etc [98-100].

- Hot and dilute plasmas $\left(T \geq 10^{6}{ }^{\circ} \mathrm{K}, n_{e} \sim 10^{14} / \mathrm{cm}^{3}\right)$, high-temperature thermonuclear fusion via magnetic confinement devices e.g. Tokamaks, Stellarators, etc $[97,101,102]$

- Heavy ion interactions with matter [92,103-106]

- Hadron therapy by high-energy ( $300 \mathrm{MeV} / \mathrm{amu}$ ) light ions (from proton to Carbon nuclei) for treatment of deep-seated tumors in patients at either physics-based facilities or at hospital-built dedicated accelerators in several countries (USA, Germany, France, Austria, Sweden, Italy, Japan, Russia, ...) [107-124].

Atomic units shall be used explicitly unless stated otherwise.

\section{General theory with and without the distorted wave formalism}

\subsection{Coulomb-modified initial and final scattering states without distorted waves}

One of the most important problems for testing theories in pure four-particle ionatom collisions is double charge exchange (double electron transfer, double electron capture). Here, two electrons $e_{1}$ and $e_{2}$, that are initially bound to the target nucleus (T), both end up finally in another bound state, but this time around the projectile nucleus $(\mathrm{P})$. This process is symbolized by:

$$
Z_{\mathrm{P}}+\left(Z_{\mathrm{T}} ; e_{1}, e_{2}\right)_{i} \longrightarrow\left(Z_{\mathrm{P}} ; e_{1}, e_{2}\right)_{f}+Z_{\mathrm{T}}
$$

or equivalently,

$$
\mathrm{P}+\left(\mathrm{T} ; e_{1}, e_{2}\right)_{i} \longrightarrow\left(\mathrm{P} ; e_{1}, e_{2}\right)_{f}+\mathrm{T}
$$

where the parentheses indicate the bound states, whereas $Z_{\mathrm{P}}$ and $Z_{\mathrm{T}}$ are the nuclear charges of $\mathrm{P}$ and T. Let $\boldsymbol{x}_{k}$ and $\boldsymbol{s}_{k}$ be the position vectors of the $k$ th electron $e_{k}$ relative to $\mathrm{T}$ and $\mathrm{P}$, respectively $(k=1,2)$. Further, let $R$ be the inter-nuclear axis with $\boldsymbol{R}$ being the position vector of $\mathrm{P}$ relative to $\mathrm{T}(R=|\boldsymbol{R}|)$. We denote by $\boldsymbol{r}_{i}$ or $\boldsymbol{r}_{f}$ the position vector of $\mathrm{P}$ or $\mathrm{T}$ relative to the center-of-mass of $\left(Z_{\mathrm{T}} ; e_{1}, e_{2}\right)_{i}$ or $\left(Z_{\mathrm{P}} ; e_{1}, e_{2}\right)_{f}$. The elements of the set $\left\{\boldsymbol{r}_{i}, \boldsymbol{r}_{f}, \boldsymbol{x}_{1,2}, \boldsymbol{s}_{1,2}\right\}$ can be connected to each other by introducing the position vectors $\left\{\boldsymbol{r}_{\mathrm{P}}, \boldsymbol{r}_{\mathrm{T}}, \boldsymbol{r}_{1,2}\right\}$ of $\left\{\mathrm{P}, \mathrm{T}, e_{1,2}\right\}$ relative to the origin $\mathrm{O}$ of an arbitrary Galilean reference frame XOYZ. This gives the defining expressions for $\left\{\boldsymbol{x}_{1,2}, \boldsymbol{s}_{1,2}, \boldsymbol{r}_{i}, \boldsymbol{r}_{f}\right\}$ as well as for the vectors of the inter-nuclear $(\boldsymbol{R})$ and inter-electronic $\left(\boldsymbol{r}_{12}\right)$ distances:

$$
\left.\begin{array}{l}
\boldsymbol{x}_{1,2}=\boldsymbol{r}_{1,2}-\boldsymbol{r}_{\mathrm{T}}, \quad \boldsymbol{s}_{1,2}=\boldsymbol{r}_{1,2}-\boldsymbol{r}_{\mathrm{P}} \\
\boldsymbol{R}=\boldsymbol{r}_{\mathrm{P}}-\boldsymbol{r}_{\mathrm{T}}, \quad \boldsymbol{r}_{12}=\boldsymbol{r}_{1}-\boldsymbol{r}_{2} \\
\boldsymbol{r}_{i}=\boldsymbol{r}_{\mathrm{P}}-\frac{M_{\mathrm{T}} \boldsymbol{r}_{\mathrm{T}}+\boldsymbol{r}_{1}+\boldsymbol{r}_{2}}{M_{\mathrm{T}}+2}, \quad \boldsymbol{r}_{f}=\boldsymbol{r}_{\mathrm{T}}-\frac{M_{\mathrm{P}} \boldsymbol{r}_{\mathrm{P}}+\boldsymbol{r}_{1}+\boldsymbol{r}_{2}}{M_{\mathrm{P}}+2}
\end{array}\right\},
$$


where $M_{\mathrm{K}}$ is the mass of the Kth nucleus $(\mathrm{K}=\mathrm{P}, \mathrm{T})$. Here, the electron mass $m_{e}$ does not explicitly appear since $m_{e}=1$ in atomic units. The position vectors introduced in (2.3) are connected to each other by the relations:

$$
\left.\begin{array}{l}
\boldsymbol{R}=x_{1}-s_{1}=x_{2}-s_{2} \\
x_{12}=x_{1}-x_{2}, \quad s_{12}=s_{1}-s_{2}, \quad x_{12}=s_{12} \equiv r_{12} \\
r_{i}=-b_{1} r_{f}-\frac{a_{1}}{\mu_{f}}\left(s_{1}+s_{2}\right), \quad r_{f}=-a_{1} r_{i}-\frac{b_{1}}{\mu_{i}}\left(x_{1}+x_{2}\right)
\end{array}\right\},
$$

where $\mu_{i}$ and $\mu_{f}$ are the reduced mass of $\mathrm{P}+\left(\mathrm{T} ; e_{1}, e_{2}\right)_{i}$ and $\left(\mathrm{P} ; e_{1}, e_{2}\right)_{f}+\mathrm{T}$, respectively

$$
\left.\begin{array}{l}
b_{1}=\frac{M_{\mathrm{T}}}{M_{\mathrm{T}}+1}, \quad b_{2}=\frac{M_{\mathrm{T}}+1}{M_{\mathrm{T}}+2}, \quad \mu_{i}=\frac{M_{\mathrm{P}}\left(M_{\mathrm{T}}+2\right)}{M_{\mathrm{P}}+M_{\mathrm{T}}+2} \\
a_{1}=\frac{M_{\mathrm{P}}}{M_{\mathrm{P}}+1}, \quad a_{2}=\frac{M_{\mathrm{P}}+1}{M_{\mathrm{P}}+2}, \quad \mu_{f}=\frac{M_{\mathrm{T}}\left(M_{\mathrm{P}}+2\right)}{M_{\mathrm{T}}+M_{\mathrm{P}}+2}
\end{array}\right\} .
$$

For a subsequent derivation, it is useful to decompose the vector $\boldsymbol{R}$ of the inter-nuclear axis $R$ into its two orthogonal vectorial components:

$$
\boldsymbol{R}=\{\boldsymbol{\rho}, \boldsymbol{Z}\}, \boldsymbol{R}=\boldsymbol{\rho}+\boldsymbol{Z}, \boldsymbol{\rho} \cdot \boldsymbol{Z}=0, \mathrm{~d} \boldsymbol{R}=\mathrm{d} \rho \mathrm{d} Z \text { (Volume element). }
$$

Here, the vectorial projections of $\boldsymbol{R}$ onto the $X O Y$ plane and the $Z$-axis are denoted by $\rho$ and $Z$. Both the light (electrons) and heavy (nuclei) particles are going to be presently described by fully quantum-mechanical methods through the Schrödinger equations. Therefore, in (2.6), despite the resemblance, the vector $\rho$ cannot be viewed as the impact parameter $\boldsymbol{b}$ in the straight line $\boldsymbol{R}=\boldsymbol{b}+\boldsymbol{v} t$ for the classically described motion of the projectile, where $\boldsymbol{v}$ and $t$ are the incident velocity and time, respectively. Nevertheless, using the final expressions for the full quantum-mechanical eikonal transition amplitudes, we will extract (by means of the Fourier integrals) their semiclassical impact parameter dependent counterparts. This does not mean that the eikonal version of the quantum-mechanical formalism should obviate the need for the impact parameter framework. Quite the contrary, the four-body impact parameter formulations and the four-body full quantum-mechanical formalisms should be treated on the same footing for ion-atom collisions involving heavy nuclei. The reason is in the dualism as it is always reassuring to obtain the same results from two different types of descriptions of the same problem. This dualism stems from the eikonal setting (with heavy mass limits and predominantly forward scattering of projectiles) which is the basis of the equivalence between the fully quantum-mechanical and the semi-classical impact parameter developments (both in the four-body formulations) for double charge exchange processes. 
The complete Schrödinger equation describing all the states of the whole system is given by:

$$
(H-E) \Psi=0,
$$

where $H$ is the full Hamiltonian operator and $E$ is the total energy

$$
\begin{aligned}
E & =E_{i}=E_{f} \quad(\text { Energy conservation law }) ; \\
E_{i} & =\frac{k_{i}^{2}}{2 \mu_{i}}+\epsilon_{i}^{\mathrm{T}}, E_{f}=\frac{k_{f}^{2}}{2 \mu_{f}}+\epsilon_{f}^{\mathrm{P}} .
\end{aligned}
$$

Here, $\boldsymbol{k}_{i}$ and $\boldsymbol{k}_{f}$ are the initial and final wave vectors, whereas $\epsilon_{i}^{\mathrm{T}}$ and $\epsilon_{f}^{\mathrm{P}}$ are the binding energies of the two electrons around the target and projectile nucleus in the entrance and exit channels, respectively. Using the momentum vectors $\boldsymbol{k}_{i, f}$ and and the reduced masses $\mu_{i, f}$, the initial and final velocity vectors $\boldsymbol{v}_{i, f}$ and their unit vectors $\hat{\boldsymbol{v}}_{i, f}$ are:

$$
\boldsymbol{v}_{i, f}=\frac{\boldsymbol{k}_{i, f}}{\mu_{i, f}}, \quad \hat{\boldsymbol{v}}_{i, f}=\frac{\boldsymbol{v}_{i, f}}{v_{i, f}}, \quad \hat{\boldsymbol{k}}_{i, f}=\frac{\boldsymbol{k}_{i, f}}{k_{i, f}} .
$$

Hereafter, we will choose the initial velocity along the $Z$-axis, so that $\boldsymbol{v}_{i}=\left(0,0, v_{i}\right)$. Taking the target atomic system to be at rest, the relative velocity vector of the colliding particles becomes equal to the incident velocity of the projectile nucleus $\mathrm{P}$. Thus, the incident velocity of $\mathrm{P}$ is equal to $\boldsymbol{v}_{i}$.

Using the energy conservation law $k_{i}^{2} /\left(2 \mu_{i}\right)+\epsilon_{i}^{\mathrm{T}}=k_{f}^{2} /\left(2 \mu_{f}\right)+\epsilon_{f}^{\mathrm{P}}$ from (2.8), the following exact relationship is obtained between the magnitudes $v_{f}=\left|\boldsymbol{v}_{f}\right|$ and $v_{i}=\left|\boldsymbol{v}_{i}\right|$ of the initial and final velocity vectors $\boldsymbol{v}_{f}$ and $\boldsymbol{v}_{i}$ :

$$
v_{f}=v_{i} \sqrt{\frac{\mu_{i}}{\mu_{f}}\left[1+\frac{\epsilon_{i}^{\mathrm{T}}-\epsilon_{f}^{\mathrm{P}}}{k_{i}^{2} /\left(2 \mu_{i}\right)}\right]}
$$

The total Hamiltonian $H$ is defined by:

$$
H=H_{0}+V,
$$

where $V$ is the complete interaction potential

$$
\begin{aligned}
& V=V_{\mathrm{T} 1}+V_{\mathrm{T} 2}+V_{\mathrm{P} 1}+V_{\mathrm{P} 2}+V_{12}+V_{\mathrm{PT}}, \\
& V_{\mathrm{T} k}=-\frac{Z_{\mathrm{T}}}{x_{k}}, \quad V_{\mathrm{P} k}=-\frac{Z_{\mathrm{P}}}{s_{k}}(k=1,2), \quad V_{12}=\frac{1}{r_{12}}, \quad V_{\mathrm{PT}}=\frac{Z_{\mathrm{P}} Z_{\mathrm{T}}}{R} .
\end{aligned}
$$

The quantity $H_{0}$ is the full kinetic energy operator which takes two equivalent forms in the two sets of the independent variables $\left\{\boldsymbol{r}_{i}, \boldsymbol{x}_{1}, \boldsymbol{x}_{2}\right\}$ and $\left\{\boldsymbol{r}_{f}, \boldsymbol{s}_{1}, \boldsymbol{s}_{2}\right\}$ :

$$
\left\{\boldsymbol{r}_{i}, \boldsymbol{x}_{1}, \boldsymbol{x}_{2}\right\}: \quad H_{0}=K_{i}-\frac{1}{2 b_{1}} \nabla_{x_{1}}^{2}-\frac{1}{2 b_{1}} \nabla_{x_{2}}^{2}-\frac{1}{M_{\mathrm{T}}} \nabla_{\boldsymbol{x}_{1}} \cdot \nabla_{\boldsymbol{x}_{2}},
$$




$$
\left\{\boldsymbol{r}_{f}, \boldsymbol{s}_{1}, \boldsymbol{s}_{2}\right\}: \quad H_{0}=K_{f}-\frac{1}{2 a_{1}} \nabla_{s_{1}}^{2}-\frac{1}{2 a_{1}} \nabla_{s_{2}}^{2}-\frac{1}{M_{\mathrm{P}}} \nabla_{s_{1}} \cdot \nabla_{s_{2}}
$$

where $K_{i, f}$ are the kinetic energy operators of the relative motions of heavy particles

$$
K_{i}=-\frac{1}{2 \mu_{i}} \nabla_{r_{i}}^{2}, \quad K_{f}=-\frac{1}{2 \mu_{f}} \nabla_{r_{f}}^{2}
$$

\subsubsection{Eikonal formalism for dominant forward scatterings of heavy particles}

Fast heavy particles only slightly deviate from their incident direction. Consequently, total cross sections are dominated by forward scattering. This justifies the use of the eikonal variant of the full quantum-mechanical treatment. For such collisions, the eikonal formalism consists of a sequence of the following relations:

$$
\begin{aligned}
& \text { Eikonal formalism : } \\
& M_{\mathrm{P}, \mathrm{T}} \gg 1, \quad k_{i, f} \gg 1, \quad k_{i, f}^{2} /\left(2 \mu_{i, f}\right) \gg \max \left(\left|\epsilon_{i}^{\mathrm{T}}\right|,\left|\epsilon_{f}^{\mathrm{P}}\right|\right) \\
& \hat{\boldsymbol{k}}_{f} \approx \hat{\boldsymbol{k}}_{i}\left(\hat{\boldsymbol{v}}_{f} \approx \hat{\boldsymbol{v}}_{i} \equiv \hat{\boldsymbol{v}}\right) \quad(\text { Forward scattering }) \\
& \boldsymbol{r}_{i} \approx \boldsymbol{R}, \quad \boldsymbol{r}_{f} \approx-\boldsymbol{R} \\
& k_{i} r_{i}-\boldsymbol{k}_{i} \cdot \boldsymbol{r}_{i} \approx \mu(v R-\boldsymbol{v} \cdot \boldsymbol{R}), \quad k_{f} r_{f}-\boldsymbol{k}_{f} \cdot \boldsymbol{r}_{f} \approx \mu(v R+\boldsymbol{v} \cdot \boldsymbol{R}) \\
& \text { Neglect of the mass polarizations : } M_{\mathrm{T}}^{-1} \nabla_{x_{1}} \cdot \nabla_{x_{2}}, \quad M_{\mathrm{P}}^{-1} \nabla_{s_{1}} \cdot \nabla_{s_{2}} \\
& \text { Linearization of } K_{i, f} \text { via : } K_{i, f} \approx K_{i, f}^{(\mathrm{eik})} \equiv k_{i, f}^{2} /\left(2 \mu_{i, f}\right)-\boldsymbol{v}_{i, f} \cdot\left(\boldsymbol{k}_{i, f} \pm i \nabla_{r_{i, f}}\right) \text { ) }
\end{aligned}
$$

where $\mu$ is the reduced mass of the two nuclei, $\mu=M_{\mathrm{P}} M_{\mathrm{T}} /\left(M_{\mathrm{P}}+M_{\mathrm{T}}\right)$. Throughout the present analysis, in the entrance channel, we adopt the standard notation by which the wave vector $\boldsymbol{k}_{i}$ is the initial momentum of the projectile nucleus $\mathrm{P}$ with respect to $\left(T ; e_{1}, e_{2}\right)_{i}$ for the process $(2.1)$. However, in the exit channel, we use the the nonstandard notation where the wave vector $\boldsymbol{k}_{f}$ is the final momentum of $\left(P ; e_{1}, e_{2}\right)_{f}$ with respect to the target nucleus T. In other words, the non-standard initial wave vector $\left\{\boldsymbol{k}_{f}\right\}_{\text {non-standard }}$ changes the direction of its standard counterpart $\left\{\boldsymbol{k}_{f}\right\}_{\text {standard }}$ i.e. $\left\{\boldsymbol{k}_{f}\right\}_{\text {non-standard }}=-\left\{\boldsymbol{k}_{f}\right\}_{\text {standard }}$. Here, $\left\{\boldsymbol{k}_{f}\right\}_{\text {standard }}$ is the the usual momentum vector of the target nucleus $\mathrm{T}$ with respect to the newly formed heliumlike atomic system $\left(P ; e_{1}, e_{2}\right)_{f}$. The reason for reversing the sign of $\left\{\boldsymbol{k}_{f}\right\}_{\text {standard }}$ in $\left\{\boldsymbol{k}_{f}\right\}_{\text {non-standard }}$ is in the fact that for charge exchange in heavy ion-atom collisions, the final velocity vector $\boldsymbol{v}_{f}$ is very close to the initial velocity vector $\boldsymbol{v}_{i}$. This yields a convenient notation in (2.17) via $\boldsymbol{v}_{f} \approx \boldsymbol{v}_{i} \equiv \boldsymbol{v}$ which, as one of the signatures of the eikonal formalism for heavy particles, makes in evidence the dominant contribution of scattering in 
the forward direction $\left(\hat{\boldsymbol{v}}_{i, f} \approx \hat{\boldsymbol{v}}_{i, f} \equiv \hat{\boldsymbol{v}}\right)$. Moreover, the same type of the relation $v_{f} \approx v_{i} \equiv v$ also exists between the absolute values $v_{f}$ and $v_{i}$ of the velocity vectors $\boldsymbol{v}_{f}$ and $\boldsymbol{v}_{i}$ as can be seen by applying $k_{i, f}^{2} /\left(2 \mu_{i, f}\right) \gg \max \left(\left|\epsilon_{i}^{\mathrm{T}}\right|,\left|\epsilon_{f}^{\mathrm{P}}\right|\right)$ from (2.17) to (2.10). Thus, we see that, as stated, the vectors $\boldsymbol{v}_{f}$ and $\boldsymbol{v}_{i}$ are close to each other in the sense of being practically collinear:

$$
\hat{\boldsymbol{v}}_{i, f} \approx \hat{\boldsymbol{v}}_{i, f} \equiv \hat{\boldsymbol{v}}, \quad v_{f} \approx v_{i} \equiv v \quad \therefore \boldsymbol{v}_{f} \approx \boldsymbol{v}_{i} \equiv \boldsymbol{v}
$$

There is yet another advantage of using the eikonal hypothesis, since the small scattering angles of heavy projectiles imply:

$$
\left.\begin{array}{l}
\boldsymbol{k}_{i} \cdot \boldsymbol{r}_{i}+\boldsymbol{k}_{f} \cdot \boldsymbol{r}_{f}=2 \boldsymbol{\alpha} \cdot\left(\boldsymbol{s}_{1}+\boldsymbol{s}_{2}\right)+2 \boldsymbol{\beta} \cdot\left(\boldsymbol{x}_{1}+\boldsymbol{x}_{2}\right) \\
\boldsymbol{k}_{i} \cdot \boldsymbol{r}_{i}+\boldsymbol{k}_{f} \cdot \boldsymbol{r}_{f}=2 \boldsymbol{\beta} \cdot \boldsymbol{R}-\boldsymbol{v} \cdot\left(\boldsymbol{s}_{1}+\boldsymbol{s}_{2}\right)=-2 \boldsymbol{\alpha} \cdot \boldsymbol{R}-\boldsymbol{v} \cdot\left(\boldsymbol{x}_{1}+\boldsymbol{x}_{2}\right) \\
\mathbf{2} \alpha=\boldsymbol{\eta}-v^{+} \hat{\boldsymbol{v}}, \mathbf{2} \beta=-\boldsymbol{\eta}-v^{-} \hat{\boldsymbol{v}}, \boldsymbol{\alpha}+\boldsymbol{\beta}=-\boldsymbol{v}, v^{ \pm}=v \pm \frac{\epsilon_{f}^{\mathrm{P}}-\epsilon_{i}^{\mathrm{T}}}{v}
\end{array}\right\} .
$$

Here, $\boldsymbol{\eta}$, as the transversal, two-dimensional, vectorial component of the momentum transfer vector $\boldsymbol{k}_{f}-\boldsymbol{k}_{i}$, is given by:

$$
\boldsymbol{\eta}=\left(\eta \cos \phi_{\eta}, \eta \sin \phi_{\eta}, 0\right), \boldsymbol{\eta} \cdot \boldsymbol{v}=0, \boldsymbol{\rho} \cdot \boldsymbol{v}=0
$$

With the neglect of the mass polarization terms $\left(1 / M_{\mathrm{T}}\right) \boldsymbol{\nabla}_{\boldsymbol{x}_{1}} \cdot \nabla_{\boldsymbol{x}_{2}}$ and $\left(1 / M_{\mathrm{P}}\right) \boldsymbol{\nabla}_{\boldsymbol{s}_{1}} \cdot \boldsymbol{\nabla}_{\boldsymbol{s}_{2}}$ in (2.14) and (2.15), respectively, the kinetic energy operator $H_{0}$ becomes:

$$
H_{0}=-\frac{1}{2 \mu_{i}} \nabla_{r_{i}}^{2}+H_{0 \mathrm{~T}}=K_{i}+H_{0 \mathrm{~T}},
$$

or alternatively

$$
H_{0}=-\frac{1}{2 \mu_{f}} \nabla_{r_{f}}^{2}+H_{0 \mathrm{P}}=K_{f}+H_{0 \mathrm{P}}
$$

where

$$
\begin{gathered}
H_{0 \mathrm{~T}}=-\frac{1}{2 b_{1}} \nabla_{x_{1}}^{2}-\frac{1}{2 b_{2}} \nabla_{x_{2}}^{2}, \\
H_{0 \mathrm{P}}=-\frac{1}{2 a_{1}} \nabla_{s_{1}}^{2}-\frac{1}{2 a_{2}} \nabla_{s_{2}}^{2} .
\end{gathered}
$$

In (2.21) and (2.22), even though we will adopt the eikonal hypothesis throughout, we provisionally employ, for convenience, the exact operator $K_{i, f}$ instead of $K_{i, f}^{\text {(eik) }}$ for the relative motions of heavy particles. However, in the consistent eikonal formalism (2.17), either the exact kinetic energy operators $K_{i, f}=\nabla_{r_{i, f}}^{2} /\left(2 \mu_{i, f}\right)$ or their eikonal, linearized forms $K_{i, f}^{(\text {eik })}=k_{i, f}^{2} /\left(2 \mu_{i, f}\right)-\boldsymbol{v}_{i, f} \cdot\left(\boldsymbol{k}_{i, f} \pm i \nabla_{r_{i, f}}\right)$ can equivalently be used. 
For the given Coulombic potentials $Z_{i, f} / r_{i, f}$, the Schrödinger equations with $K_{i, f}$ and $K_{i, f}^{(\text {eik })}$ give the full Coulomb wave functions $F_{i, f}^{ \pm}\left(\boldsymbol{r}_{i, f}\right)$ and their logarithmic phase factors $F_{i, f}^{(\mathrm{eik}) \pm}\left(\boldsymbol{r}_{i, f}\right)$, respectively. The differences in the results for the total cross sections based upon the alternative pairs $\left\{K_{i, f}, F_{i, f}^{ \pm}\left(\boldsymbol{r}_{i, f}\right)\right\}$ and $\left\{K_{i, f}^{(\mathrm{eik})}, F_{i, f}^{(\mathrm{eik}) \pm}\left(\boldsymbol{r}_{i, f}\right)\right\}$ are negligibly small, being of the order of or less than $1 / \mu_{i, f}$ and, as such, altering merely the 3 rd or 4 th decimal places, at most. Theoretically, an explicit use of $K_{i, f}^{(\text {eik) }}$ is particularly convenient when showing that the inter-nuclear potential $V_{\mathrm{PT}}$ does not contribute to the eikonal total cross sections computed from the eikonal version of the quantum-mechanical transition amplitudes in any method with the correct boundary conditions. This has first been shown in Ref. [87] for single capture processes, and it will also be presently demonstrated for double capture in rearrangement collisions of heavy nuclei and atomic target systems.

The Schrödinger equation (2.7) is to be solved subject to the physical boundary conditions associated with the scattering problem (2.1). These boundary conditions must provide the full wave function $\Psi$ with the proper outgoing $\Psi_{i}^{+}$and incoming $\Psi_{f}^{-}$spherical scattered waves at large values of the inter-aggregate separations $r_{i}$ and $r_{f}$ in the entrance and exit channel:

$$
\begin{aligned}
& \Psi_{i}^{+} \underset{r_{i} \rightarrow \infty}{\longrightarrow} \Phi_{i}^{+}, \quad \Phi_{i}^{+}=\Phi_{i} \mathrm{e}^{i v_{i} \ln \left(k_{i} r_{i}-\boldsymbol{k}_{i} \cdot \boldsymbol{r}_{i}\right)} \underset{M_{\mathrm{P}, \mathrm{T}} \gg 1}{\widetilde{\widetilde{T}}} \Phi_{i} \mathrm{e}^{i v_{i} \ln \mu(v R-\boldsymbol{v} \cdot \boldsymbol{R})}, \\
& \Psi_{f}^{-} r_{f \rightarrow \infty} \Phi_{f}^{-}, \quad \Phi_{f}^{-}=\Phi_{f} \mathrm{e}^{-i v_{f} \ln \left(k_{f} r_{f}-\boldsymbol{k}_{f} \cdot \boldsymbol{r}_{f}\right)}{ }_{M_{\mathrm{P}, \mathrm{T}}>1} \Phi_{f} \mathrm{e}^{-i v_{f} \ln \mu(v R+\boldsymbol{v} \cdot \boldsymbol{R})},
\end{aligned}
$$

where

$$
v_{i}=\frac{Z_{\mathrm{P}}\left(Z_{\mathrm{T}}-2\right)}{v}, \quad v_{f}=\frac{Z_{\mathrm{T}}\left(Z_{\mathrm{P}}-2\right)}{v} .
$$

Here, $\Phi_{i}$ and $\Phi_{f}$ are the initial and final unperturbed states:

$$
\begin{aligned}
& \Phi_{i}=\varphi_{i}\left(\boldsymbol{x}_{1}, \boldsymbol{x}_{2}\right) \mathrm{e}^{i \boldsymbol{k}_{i} \cdot \boldsymbol{r}_{i}}, \\
& \Phi_{f}=\varphi_{f}\left(\boldsymbol{s}_{1}, \boldsymbol{s}_{2}\right) \mathrm{e}^{-i \boldsymbol{k}_{f} \cdot \boldsymbol{r}_{f}},
\end{aligned}
$$

where $\varphi_{i}\left(\boldsymbol{x}_{1}, \boldsymbol{x}_{2}\right)$ and $\varphi_{f}\left(\boldsymbol{s}_{1}, \boldsymbol{s}_{2}\right)$ are the bound state wave functions of atomic systems $\left(Z_{\mathrm{T}} ; e_{1}, e_{2}\right)_{i}$ and $\left(Z_{\mathrm{P}} ; e_{1}, e_{2}\right)_{f}$. These latter wave functions satisfy the equations:

$$
\begin{gathered}
\left(H_{0 \mathrm{~T}}+V_{\mathrm{T}}-\epsilon_{i}^{\mathrm{T}}\right) \varphi_{i}\left(\boldsymbol{x}_{1}, \boldsymbol{x}_{2}\right)=0, \\
\left(H_{0 \mathrm{P}}+V_{\mathrm{P}}-\epsilon_{f}^{\mathrm{P}}\right) \varphi_{f}\left(\boldsymbol{s}_{1}, \boldsymbol{s}_{2}\right)=0,
\end{gathered}
$$

with

$$
\begin{gathered}
V_{\mathrm{T}}=V_{\mathrm{T} 1}+V_{\mathrm{T} 2}+V_{12}, \\
V_{\mathrm{P}}=V_{\mathrm{P} 1}+V_{\mathrm{P} 2}+V_{12} .
\end{gathered}
$$


The particular forms of the channel states $\Phi_{i}^{+}$and $\Phi_{f}^{-}$from Eqs. (2.25) and (2.26) account for all the long-range distortion Coulomb effects due to the residual interactions $V_{i, f}^{\infty}$ between the two scattering particles:

$$
V_{i}^{\infty}=\frac{Z_{\mathrm{P}}\left(Z_{\mathrm{T}}-2\right)}{r_{i}}, \quad V_{f}^{\infty}=\frac{Z_{\mathrm{T}}\left(Z_{\mathrm{P}}-2\right)}{r_{f}} .
$$

According to (2.17), within the eikonal mass limit $M_{\mathrm{P}, \mathrm{T}} \gg 1$, the relations $\boldsymbol{r}_{i, f} \approx \pm \boldsymbol{R}$ are amply justified, and this implies:

$$
V_{i}^{\infty} \underset{M_{\mathrm{T}} \gg 1}{\longrightarrow} V_{i, \mathrm{eik}}^{\infty}, \quad V_{f}^{\infty} \underset{M_{\mathrm{P}} \gg 1}{\longrightarrow} V_{f, \text { eik }}^{\infty}
$$

where

$$
V_{i, \mathrm{eik}}^{\infty}=\frac{Z_{\mathrm{P}}\left(Z_{\mathrm{T}}-2\right)}{R}, \quad V_{f, \mathrm{eik}}^{\infty}=\frac{Z_{\mathrm{T}}\left(Z_{\mathrm{P}}-2\right)}{R}
$$

As such, the distorting potentials $V_{i, f}^{\infty}$ represent the correct asymptotic behaviors of the corresponding initial and final perturbations $V_{i, f}$, which are introduced by:

$$
\begin{aligned}
V_{i} & =V_{\mathrm{P} 1}+V_{\mathrm{P} 2}+V_{\mathrm{PT}_{r_{i}} \rightarrow \infty} V_{i}^{\infty}, \\
V_{f} & =V_{\mathrm{T} 1}+V_{\mathrm{T} 2}+V_{\mathrm{PT}_{r}} \underset{r_{f}}{\longrightarrow} V_{f}^{\infty} .
\end{aligned}
$$

The unperturbed channel states $\Phi_{i}$ and $\Phi_{f}$ from Eqs. (2.28) and (2.29) satisfy the following equations:

$$
\begin{gathered}
\left(H_{i}-E_{i}\right) \Phi_{i}=0, \\
\left(H_{f}-E_{i}\right) \Phi_{f}=0,
\end{gathered}
$$

where $H_{i}$ and $H_{f}$ are the channel Hamiltonians

$$
\begin{aligned}
H_{i} & =H-V_{i}=H_{0}+V_{\mathrm{P}}, \\
H_{f} & =H-V_{f}=H_{0}+V_{\mathrm{T}} .
\end{aligned}
$$

In the eikonal approximation, the asymptotic channel states $\Phi_{i}^{+}$and $\Phi_{i}^{-}$from Eqs. (2.25) and (2.26) are the solutions of the equations:

$$
\begin{gathered}
\left(H_{i}+V_{i}^{\infty}-E_{i}\right) \Phi_{i}^{+}=0, \\
\left(H_{f}+V_{f}^{\infty}-E_{f}\right) \Phi_{f}^{-}=0 .
\end{gathered}
$$

For scattering, the complete Schrödinger equation (2.7) for the original problem is written in the forms that emphasize the appropriate boundary conditions:

$$
\begin{aligned}
& \left(H-E_{i}\right) \Psi_{i}^{+}=0, \\
& \left(H-E_{f}\right) \Psi_{f}^{-}=0 .
\end{aligned}
$$




\subsubsection{The prior and post forms of the transition amplitudes}

It is convenient to introduce the initial and final compound wave functions $\Upsilon_{i, f}^{ \pm}$ (hereafter called the initial and final scattering amalgamates) as the images of the applications of the perturbations $V_{i, f}-V_{i, f}^{\infty}$ onto the Coulomb-modified channels states $\Phi_{i, f}^{ \pm}$:

$$
\Upsilon_{i}^{+}=\left(V_{i}-V_{i}^{\infty}\right) \Phi_{i}^{+}, \quad \Upsilon_{f}^{-}=\left(V_{f}-V_{f}^{\infty}\right) \Phi_{f}^{-}
$$

Then the prior form of the full transition amplitude $T_{i f}^{-}$is obtained by projecting the initial scattering amalgamate $\Upsilon_{i}^{+}$onto the final complete scattering state $\Psi_{f}^{-}$. This projection is the overlap integral between $\Upsilon_{i}^{+}$and $\Psi_{f}^{-}$:

$$
\text { Prior : } \quad T_{i f}^{-}=\left\langle\Psi_{f}^{-} \mid \Upsilon_{i}^{+}\right\rangle \text {. }
$$

Likewise, the post form of the full transition amplitude $T_{i f}^{+}$is the scalar or inner product between the final scattering amalgamate $\Upsilon_{f}^{-}$and the initial complete scattering state $\Psi_{i}^{+}$:

$$
\text { Post : } \quad T_{i f}^{+}=\left\langle\Upsilon_{f}^{-} \mid \Psi_{i}^{+}\right\rangle \text {. }
$$

Overall, for a straightforward book keeping, the ansatz $\Upsilon_{i}^{+}$from the prior form $T_{i f}^{-}$ fuses the Coulomb-modified initial state $\Phi_{i}^{+}$and the corresponding perturbation interaction $V_{i}-V_{i}^{\infty}$ via $\Upsilon_{i}^{+}=\left(V_{i}-V_{i}^{\infty}\right) \Phi_{i}^{+}$. Similarly, and for the same reason, the ansatz $\Upsilon_{f}^{-}$from the post form $T_{i f}^{+}$merges the Coulomb-modified final state vector $\Phi_{f}^{-}$ and the associated perturbation interaction $V_{f}-V_{f}^{\infty}$ as $\Upsilon_{f}^{-}=\left(V_{f}-V_{f}^{\infty}\right) \Phi_{f}^{-}$. In the prior/post transition amplitudes $T_{i f}^{\mp}$ from (2.48) and (2.49), the asymptotic Coulomb potentials $V_{i, f}^{\infty}$ must always be subtracted from the perturbations $V_{i, f}$ to accommodate for the difference between $\Phi_{i, f}^{ \pm}$and $\Phi_{i, f}$. Such couplings of $\Phi_{i, f}^{ \pm}$and $V_{i, f}-V_{i, f}^{\infty}$ embedded in the state vectors $\Upsilon_{i, f}^{ \pm}$emphasize the fact that the perturbation potentials and the channel states must systematically be consistent with each other. Any change in $\Phi_{i, f}^{ \pm}$ought to be accompanied by the appropriate alteration of $V_{i, f}-V_{i, f}^{\infty}$ and vice verse.

In the eikonal mass limit $M_{\mathrm{P}, \mathrm{T}} \gg 1$, the relations (2.35) and (2.36) imply that the perturbation interactions $V_{i}-V_{i}^{\infty}$ and $V_{f}-V_{f}^{\infty}$ from the transition amplitudes (2.48) and (2.49) do not contain the inter-nuclear potential $V_{\mathrm{PT}}$ :

$$
\begin{aligned}
& V_{i}-V_{i}^{\infty}=V_{\mathrm{P} 1}+V_{\mathrm{P} 2}+V_{\mathrm{PT}}-V_{i}^{\infty} \\
& \underset{M_{\mathrm{T}} \gg 1}{\longrightarrow} V_{\mathrm{P} 1}+V_{\mathrm{P} 2}+V_{\mathrm{PT}}-V_{i, \mathrm{eik}}^{\infty} \\
&=Z_{\mathrm{P}}\left(\frac{2}{R}-\frac{1}{s_{1}}-\frac{1}{s_{2}}\right)
\end{aligned}
$$




$$
\begin{aligned}
V_{f}-V_{f}^{\infty} & =V_{\mathrm{T} 1}+V_{\mathrm{T} 2}+V_{\mathrm{PT}}-V_{f}^{\infty} \\
& \overrightarrow{M_{\mathrm{P}} \gg 1} V_{\mathrm{T} 1}+V_{\mathrm{T} 2}+V_{\mathrm{PT}}-V_{f, \text { eik }}^{\infty} \\
& =Z_{\mathrm{T}}\left(\frac{2}{R}-\frac{1}{x_{1}}-\frac{1}{x_{2}}\right) .
\end{aligned}
$$

For large $R$ in the entrance channel, both $s_{1}$ and $s_{2}$ are also large. Similarly, $x_{1}$ and $x_{2}$ are large for large $R$ in the exit channel. This indicates that the perturbations $Z_{\mathrm{P}}\left(2 / R-1 / s_{1}-1 / s_{2}\right)$ and $Z_{\mathrm{T}}\left(2 / R-1 / x_{1}-1 / x_{2}\right)$ in the entrance and exit channels, respectively, are of short range. Indeed, the use of the Taylor expansion shows that both $Z_{\mathrm{P}}\left(2 / R-1 / s_{1}-1 / s_{2}\right)$ and $Z_{\mathrm{T}}\left(2 / R-1 / x_{1}-1 / x_{2}\right)$ are the short-range interactions:

$$
\begin{aligned}
& V_{i}-V_{i}^{\infty} \underset{M_{\mathrm{P}} \gg 1}{\longrightarrow} Z_{\mathrm{P}}\left(\frac{2}{R}-\frac{1}{s_{1}}-\frac{1}{s_{2}}\right) \underset{r_{i} \rightarrow \infty}{\longrightarrow} Z_{\mathrm{P}} \mathcal{O}\left(R^{-2}\right), \\
& V_{f}-V_{f}^{\infty} \underset{M_{\mathrm{P} \gg 1}}{\longrightarrow} Z_{\mathrm{T}}\left(\frac{2}{R}-\frac{1}{x_{1}}-\frac{1}{x_{2}}\right) \underset{r_{f} \rightarrow \infty}{\longrightarrow} Z_{\mathrm{T}} \mathcal{O}\left(R^{-2}\right) .
\end{aligned}
$$

The scattering states $\Psi_{i, f}^{ \pm}$and $\Phi_{i, f}^{ \pm}$with the same correct asymptotic behaviors (2.25) and (2.26) at large distances can also properly be connected to each other in any region with no necessary reference to the the limits $r_{i, f} \rightarrow \infty$. To this end, we define the total Møller wave operators $\Omega_{i, f}^{ \pm}$as:

$$
\Omega_{i}^{+}=1+G^{+}\left(V_{i}-V_{i}^{\infty}\right), \quad \Omega_{f}^{-}=1+G^{-}\left(V_{f}-V_{f}^{\infty}\right),
$$

where $G^{ \pm}$are the total Green operators (resolvents)

$$
G^{+}=\frac{1}{E_{i}-H+i \varepsilon}, \quad G^{-}=\frac{1}{E_{f}-H-i \varepsilon} \quad(\varepsilon>0) .
$$

Then the sought relationships between $\Psi_{i, f}^{ \pm}$and $\Phi_{i, f}^{ \pm}$at any inter-particle distance are given by:

$$
\begin{aligned}
& \left|\Psi_{i}^{+}\right\rangle=\Omega_{i}^{+}\left|\Phi_{i}^{+}\right\rangle, \\
& \left|\Psi_{f}^{-}\right\rangle=\Omega_{f}^{-}\left|\Phi_{f}^{-}\right\rangle .
\end{aligned}
$$

The expressions (2.56) and (2.57) must be consistent with the full Schrödinger equations (2.45) and (2.46). This is true as can be checked by multiplication of (2.56) and (2.57) with $E-H+i \varepsilon$ and $E-H-i \varepsilon$, in which case Eqs. (2.45) and (2.46) are obtained in the limits $\varepsilon \rightarrow 0^{+}$and $\varepsilon \rightarrow 0^{-}$, respectively:

$$
\begin{aligned}
\lim _{\varepsilon \rightarrow 0^{ \pm}}\left(E_{i, f}-H \pm i \varepsilon\right) \Psi_{i, f}^{ \pm}= & \lim _{\varepsilon \rightarrow 0^{ \pm}}\left(E_{i, f}-H \pm i \varepsilon\right) \\
& \times\left[1+\frac{1}{E_{i, f}-H \pm i \varepsilon}\left(V_{i, f}-V_{i, f}^{\infty}\right)\right] \Phi_{i, f}^{ \pm}
\end{aligned}
$$




$$
\begin{aligned}
& =\lim _{\varepsilon \rightarrow 0^{ \pm}}\left[\left(E_{i, f}-H_{i, f}-V_{i, f} \pm i \varepsilon\right)+\left(V_{i, f}-V_{i, f}^{\infty}\right)\right] \Phi_{i, f}^{ \pm} \\
& =\lim _{\varepsilon \rightarrow 0^{ \pm}}\left(E_{i, f}-H_{i, f}-V_{i, f}^{\infty} \pm i \varepsilon\right) \Phi_{i, f}^{ \pm} \\
& =\left(E_{i, f}-H_{i, f}-V_{i, f}^{\infty}\right) \Phi_{i, f}^{ \pm}=0 \quad\left(\lim _{\varepsilon \rightarrow 0^{ \pm}} i \varepsilon \Phi_{i, f}^{ \pm}=0\right),
\end{aligned}
$$

where Eqs. (2.43) and (2.44) are used, so that

$$
\lim _{\varepsilon \rightarrow 0^{ \pm}}\left(E_{i, f}-H \pm i \varepsilon\right) \Psi_{i, f}^{ \pm}=\left(E_{i, f}-H\right) \Psi_{i, f}^{ \pm}=0 \quad\left(\lim _{\varepsilon \rightarrow 0^{ \pm}} i \varepsilon \Psi_{i, f}^{ \pm}=0\right),
$$

in agreement with (2.45) and (2.46). Therefore, the scattering states $\Psi_{i, f}^{ \pm}$from (2.56) and (2.57) are indeed the solutions of the full Schrödinger equations (2.45) and (2.46). Nevertheless, the solutions (2.56) and (2.57) are formal because finding the explicit forms of $\Omega_{i, f}^{ \pm} \Phi_{i, f}^{ \pm}$for $\Psi_{i, f}^{ \pm}$is as difficult as solving the full Schrödinger equations (2.45) and (2.46). This is the case because the Møller wave operators $\Omega_{i, f}^{ \pm}$through the resolvents $G^{ \pm}$from (2.55) also contain the total Hamiltonian $H$ as do the full Schrödinger equations (2.45) and (2.46). In order to extract the physical solutions for the investigated problem, Eq. (2.7) must always be explicitly accompanied by the appropriate boundary conditions (2.25) or (2.26). This is symbolized by the superscripts \pm of the total scattering wave functions in (2.45) and (2.46). On the other hand, in the case of (2.56) and (2.57), these scattering boundary conditions are implicitly contained in the Green operators $G^{ \pm}$. This is secured by the presence of the infinitesimally small positive number $\varepsilon$ in the Green operators from (2.55). The terms $\pm \varepsilon$ in $G^{ \pm}$determine, respectively, the outgoing and incoming scattering boundary condition as per (2.25) and (2.26).

Upon substitution of (2.56) and (2.57) into (2.48) and (2.49), it follows:

$$
\begin{array}{cc}
\text { Prior : } & T_{i f}^{-}=\left\langle\Phi_{f}^{-}\left|\left\{1+\left(V_{f}-V_{f}^{\infty}\right) G^{-}\right\}^{\dagger}\left(V_{i}-V_{i}^{\infty}\right)\right| \Phi_{i}^{+}\right\rangle, \\
\text {Post : } & T_{i f}^{+}=\left\langle\Phi_{f}^{-}\left|\left(V_{f}-V_{f}^{\infty}\right)\left\{1+G^{+}\left(V_{i}-V_{i}^{\infty}\right)\right\}\right| \Phi_{i}^{+}\right\rangle,
\end{array}
$$

where $\left(V_{f}-V_{f}^{\infty}\right)^{\dagger}=V_{f}-V_{f}^{\infty}$. We emphasize that these are the $T$-matrix elements with no recourse to distorted waves and distorting potentials. The only difference relative to the conventional scattering theory with short-range interactions between the infinitely separated scattering aggregates is in the presence of the Coulomb asymptotic states $\Phi_{i, f}^{ \pm}$and the modified perturbation potentials $V_{i, f}-V_{i, f}^{\infty}$ instead of the unperturbed channel states $\Phi_{i, f}$ and the original perturbation interactions $V_{i, f}$.

\subsection{Coulomb-modified initial and final scattering states with distorted waves}

In the distorted wave formalism of scattering theory, instead of solving Eqs. (2.45) and (2.46), it is customary to consider a model problem by introducing the distorted waves $\chi_{i}^{+}$and $\chi_{f}^{-}$via the equations:

$$
\left(H_{i}+W_{i}-E_{i}\right) \chi_{i}^{+}=0,
$$




$$
\left(H_{f}+W_{f}-E_{f}\right) \chi_{f}^{-}=0,
$$

where $W_{i}$ and $W_{f}$ are certain distorting potential operators to be chosen, under the restriction that they do not cause the transition under consideration. Without any loss of generality, we can select $W_{i, f}$ to be in the additive form:

$$
W_{i}=w_{i}+V_{i}^{\infty}, \quad W_{f}=w_{f}+V_{f}^{\infty},
$$

where $w_{i, f}$ are some short-range distorting potentials. The connection of the models in (2.61) and (2.62) with the original problem in (2.45) and (2.46) is provided through the request that $\chi_{i, f}^{ \pm}$and $\Psi_{i, f}^{ \pm}$exhibit the same asymptotic behaviors as $r_{i, f} \longrightarrow \infty$ :

$$
\begin{aligned}
& \chi_{i}^{+} r_{i \rightarrow \infty} \Psi_{i}^{+} r_{i} \rightarrow \infty \\
& \chi_{f}^{-} \Phi_{r_{f} \rightarrow \infty}^{+}, \\
& \Psi_{f}^{-} \underset{r_{f} \rightarrow \infty}{\longrightarrow} \Phi_{f}^{-} .
\end{aligned}
$$

The prior $T_{i f}^{-}$and post $T_{i f}^{+}$form of the full transition amplitudes in the distorted wave theory are defined by:

$$
\begin{array}{lll}
\text { Prior : } & T_{i f}^{-}=\left\langle\Psi_{f}^{-} \mid \psi_{i}^{+}\right\rangle, & \psi_{i}^{+}=U_{i} \chi_{i}^{+}, \\
\text {Post : } & T_{i f}^{+}=\left\langle\psi_{f}^{-} \mid \Psi_{i}^{+}\right\rangle, & \psi_{f}^{-}=U_{f} \chi_{f}^{-},
\end{array}
$$

where

$$
U_{i}=V_{i}-W_{i}, \quad U_{f}=V_{f}-W_{f} .
$$

In analogy with $\Upsilon_{i, f}^{ \pm}$from (2.47), the functions $\psi_{i, f}^{ \pm}$in (2.66) and (2.67) embody the distorted waves $\chi_{i, f}^{ \pm}$and the distorting potentials $U_{i, f}$ as per $\psi_{i, f}^{ \pm}=U_{i, f} \chi_{i, f}^{ \pm}$. Here, the replacement of $\Phi_{i, f}^{ \pm}$by $\psi_{i, f}^{ \pm}$is reflected through the pertinent subtraction of $W_{i, f}$ from $V_{i, f}$.

If we add and subtract $V_{i}$ in (2.61) and use $U_{i}=V_{i}-W_{i}$ together with $H=H_{i}+V_{i}$, we can transform the Schrödinger equation for $\chi_{i}^{+}$to:

$$
\left(H-U_{i}-E_{i}\right) \chi_{i}^{+}=0 .
$$

In the same way, when adding and subtracting $V_{f}$ in (2.62), followed by identification of $V_{f}-W_{f}$ with $U_{f}$ as well as $H_{i}+V_{i}$ with $H$, we can cast the Schrödinger equation for $\chi_{f}^{-}$into the form:

$$
\left(H-U_{f}-E_{f}\right) \chi_{f}^{-}=0 .
$$

The transformed Eqs. (2.69) and (2.70) for $\chi_{i}^{+}$and $\chi_{f}^{-}$resemble the Schrödinger Eqs. (2.45) and (2.46) for the exact scattering states $\Psi_{i}^{+}$and $\Psi_{f}^{-}$, respectively. In 
fact, we have $\Psi_{i, f}^{ \pm}=\chi_{i, f}^{ \pm}$for $U_{i, f}=0$. Of course, the main interest of introducing the distorted wave theory for scattering phenomena is not in making the trivial choices $U_{i, f}=0$, as these would bring us back to the original problem with the full Schrödinger equation, which cannot be solved exactly by analytical means. Rather, an advantage and flexibility of the distorted wave scattering theory is in the possibility for making different choices of $U_{i, f} \neq 0$ that, in turn, would enable us to obtain the exact analytical solutions $\chi_{i}^{+}$and $\chi_{f}^{-}$of the model Schrödinger Eqs. (2.69) and (2.70).

Further, the scattering states $\Psi_{i, f}^{ \pm}$and $\chi_{i, f}^{ \pm}$of the original and the model problem, respectively, with the same proper asymptotes (2.64) and (2.65) can also be interrelated with no necessary reference to the regions $r_{i, f} \rightarrow \infty$. To this end, we use the total Møller wave operators $\widetilde{\Omega}_{i, f}^{ \pm}$:

$$
\widetilde{\Omega}_{i}^{+}=1+G^{+} U_{i}, \quad \widetilde{\Omega}_{f}^{-}=1+G^{-} U_{f}
$$

where $G^{ \pm}$are the same as in (2.55). Then the general connections between $\Psi_{i, f}^{ \pm}$and $\chi_{i, f}^{ \pm}$are:

$$
\begin{aligned}
& \left|\Psi_{i}^{+}\right\rangle=\widetilde{\Omega}_{i}^{+}\left|\chi_{i}^{+}\right\rangle, \\
& \left|\Psi_{f}^{-}\right\rangle=\widetilde{\Omega}_{f}^{-}\left|\chi_{f}^{-}\right\rangle .
\end{aligned}
$$

Inserting (2.72) and (2.73) into (2.66) and (2.67) we have, respectively:

$$
\begin{array}{cc}
\text { Prior : } & T_{i f}^{-}=\left\langle\chi_{f}^{-}\left|\left(1+U_{f} G^{-}\right)^{\dagger} U_{i}\right| \chi_{i}^{+}\right\rangle, \\
\text {Post : } & T_{i f}^{+}=\left\langle\chi_{f}^{-}\left|U_{f}^{\dagger}\left(1+G^{+} U_{i}\right)\right| \chi_{i}^{+}\right\rangle .
\end{array}
$$

Similarly, taking the limits $\varepsilon \longrightarrow 0^{ \pm}$, the distorted waves equations from (2.61) and (2.62) can also be formally solved as follows:

$$
\left|\chi_{i}^{+}\right\rangle=\omega_{i}^{+}\left|\Phi_{i}^{+}\right\rangle, \quad\left|\chi_{f}^{-}\right\rangle=\omega_{f}^{-}\left|\Phi_{f}^{-}\right\rangle .
$$

Here, $\omega_{i, f}^{ \pm}$are the initial and final Møller wave operators defined by:

$$
\omega_{i}^{+}=1+G_{i}^{+} w_{i}, \quad \omega_{f}^{-}=1+G_{f}^{-} w_{f}
$$

where $G_{i, f}^{ \pm}$are the initial and final Green operators

$$
G_{i}^{+}=\frac{1}{E_{i}-H_{i}-W_{i}+i \varepsilon}, \quad G_{f}^{-}=\frac{1}{E_{f}-H_{f}-W_{f}-i \varepsilon} \quad(\varepsilon>0) .
$$

One of the ways to set up a general framework for the introduction of various distorted wave approximations to the full scattering wave functions $\Psi_{i, f}^{ \pm}$consists of neglecting the total Green operators in (2.72) and (2.73). This amounts to the replacement of the 
total Møller wave operators $\tilde{\Omega}_{i, f}^{ \pm}$in $(2.72)$ and (2.73) by the unity operator $\left(\tilde{\Omega}_{i, f}^{ \pm} \approx 1\right)$ which then gives:

$$
\Psi_{i}^{+} \approx \chi_{i}^{+}, \quad \Psi_{f}^{-} \approx \chi_{f}^{-}
$$

Such choices for $\Psi_{i, f}^{ \pm}$in (2.66) and (2.67) give the first-order approximations to the full transition amplitudes that are for simplicity again denoted by the same labels $T_{i f}^{ \pm}$:

$$
\begin{array}{ll}
\text { Prior : } & T_{i f}^{-}=\left\langle\chi_{f}^{-}\left|U_{i}\right| \chi_{i}^{+}\right\rangle, \\
\text {Post : } & T_{i f}^{+}=\left\langle\chi_{f}^{-}\left|U_{f}^{\dagger}\right| \chi_{i}^{+}\right\rangle .
\end{array}
$$

In the distorted wave formalism for $T_{i f}^{ \pm}$from (2.66), (2.67), (2.80) and (2.81), the following statements define the correct boundary conditions:

$$
\left\{\begin{array}{c}
\bullet \text { Any choice of the distorted waves } \chi_{i, f}^{ \pm} \text {is permitted as long as : } \\
\bullet \text { (i) it yields the proper asymptotes (2.64) and (2.65) } \\
\bullet \text { (ii) it connects } U_{i, f} \text { and } \chi_{i, f}^{ \pm} \text {via (2.61), (2.62) and (2.68) }
\end{array}\right\} \text {. }
$$

In the next sub-sections, several choices for the tandems $\left\{U_{i, f}, \chi_{i, f}^{ \pm}\right\}$will be made yielding the known two- or one-center distorting functions with four or two electronic Coulomb waves, respectively.

\subsection{Determination of the initial and final distorted waves}

Here we shall outline the procedure of obtaining the initial and final pairs $\left\{U_{i, f}, \chi_{i, f}^{ \pm}\right\}$ in the entrance and channels.

\subsubsection{Entrance channel}

In the entrance channel, by reference to the requirement (2.64) for the correct asymptotic behavior of the initial distorted wave, the following factorized form for $\chi_{i}^{+}$is sought:

$$
\chi_{i}^{+}=\varphi_{i}\left(\boldsymbol{x}_{1}, \boldsymbol{x}_{2}\right) \zeta_{i}^{+}
$$

where $\zeta_{i}^{+}$is an unknown function. Upon inserting (2.83) into (2.61), with the help of (2.39) and $H_{0}$ from (2.14), we deduce the equation:

$$
\varphi_{i}\left(\Delta E_{i}-H_{0}-V_{i}\right) \zeta_{i}^{+}+\left\{\sum_{k=1}^{2} \frac{1}{b_{k}} \nabla_{x_{k}} \varphi_{i} \cdot \nabla_{x_{k}} \zeta_{i}^{+}+U_{i} \varphi_{i} \zeta_{i}^{+}\right\}=0
$$


where

$$
\Delta E_{i}=E_{i}-\epsilon_{i}^{\mathrm{T}}=\frac{k_{i}^{2}}{2 \mu_{i}} .
$$

We now choose the operator potential $U_{i}$ to annul the terms within the curly brackets from Eq. (2.84):

$$
U_{i}=-\sum_{k=1}^{2} \frac{1}{b_{k}} \nabla_{x_{k}} \ln \varphi_{i} \cdot \nabla_{x_{k}} .
$$

Here, the presence of $1 / \varphi_{i}$, which is implicit in the term $\nabla_{x_{k}} \ln \varphi_{i}=\left(1 / \varphi_{i}\right) \nabla_{x_{k}} \varphi_{i}$, causes no problem even for the target bound states $\varphi_{i}$ with nodes. This is the case because $U_{i}$ in (2.86) is applied to $\chi_{i}^{+}=\varphi_{i} \zeta_{i}^{+}$in which $\varphi_{i}$ is factored out in (2.83), so that:

$$
\nabla_{x_{k}} \ln \varphi_{i} \cdot \nabla_{x_{k}} \chi_{i}^{+}=\nabla_{x_{k}} \varphi_{i} \cdot \nabla_{x_{k}} \zeta_{i}^{+}
$$

Thus, $U_{i} \chi_{i}^{+}$is a regular function given by:

$$
U_{i} \chi_{i}^{+}=-\sum_{k=1}^{2} \frac{1}{b_{k}} \nabla_{x_{k}} \varphi_{i} \cdot \nabla_{x_{k}} \zeta_{i}^{+}
$$

which casts Eq. (2.84) into the form

$$
\left(\Delta E_{i}-H_{0}-V_{i}\right) \zeta_{i}^{+}=0
$$

To solve this equation, we use $H_{0}$ from (2.15). Further, in $V_{i}$ from (2.89), we use the eikonal mass limit for the $R$-dependent potential via $Z_{\mathrm{P}} Z_{\mathrm{T}} / R \approx Z_{\mathrm{P}} Z_{\mathrm{T}} / r_{f}$. Under these circumstances, the choice (2.88) provides a separation of the independent variables $\boldsymbol{r}_{f}, \boldsymbol{s}_{1}$ and $\boldsymbol{s}_{2}$ in Eq. (2.89). With such a setting, the solution of Eq. (2.89), obeying the required asymptotic behavior (2.64), is:

$$
\begin{aligned}
\zeta_{i}^{+}= & \mu_{i}^{-2 i v_{P}} \mathcal{N}^{+}\left(\nu_{\mathrm{PT}}\right)\left[N^{+}\left(\nu_{\mathrm{P}}\right)\right]^{2} \mathrm{e}^{i \boldsymbol{k}_{i} \cdot \boldsymbol{r}_{i}}{ }_{1} F_{1}\left(-i \nu_{\mathrm{PT}}, 1, i k_{i} \boldsymbol{r}_{f}+i \boldsymbol{k}_{i} \cdot \boldsymbol{r}_{f}\right) \\
& \times{ }_{1} F_{1}\left(i \nu_{\mathrm{P}}, 1, i v s_{1}+i \boldsymbol{v} \cdot \boldsymbol{s}_{1}\right){ }_{1} F_{1}\left(i \nu_{\mathrm{P}}, 1, i v s_{2}+i \boldsymbol{v} \cdot \boldsymbol{s}_{2}\right),
\end{aligned}
$$

where the symbol ${ }_{1} F_{1}$ represents the Kummer confluent hypergeometric function. The quantities $\mathcal{N}^{+}\left(\nu_{\mathrm{PT}}\right)$ and $N^{+}\left(\nu_{\mathrm{K}}\right)(\mathrm{K}=\mathrm{P}, \mathrm{T})$ are the normalization constants of the Coulomb wave functions:

$$
N^{ \pm}\left(v_{K}\right)=\mathrm{e}^{\pi v_{K} / 2} \Gamma\left(1 \pm i v_{K}\right), \mathcal{N}^{ \pm}\left(\nu_{\mathrm{PT}}\right)=\mathrm{e}^{-\pi v_{\mathrm{PT}} / 2} \Gamma\left(1 \pm i v_{\mathrm{PT}}\right)
$$


where $\Gamma$ is the Euler gamma function, with $\nu_{\mathrm{PT}}$ and $\nu_{\mathrm{K}}(\mathrm{K}=\mathrm{P}, \mathrm{T})$ being the usual Sommerfeld parameters

$$
v_{\mathrm{P}}=\frac{Z_{\mathrm{P}}}{v}, \quad v_{\mathrm{T}}=\frac{Z_{\mathrm{T}}}{v}, \quad \nu_{\mathrm{PT}}=\frac{Z_{\mathrm{P}} Z_{\mathrm{T}}}{v} .
$$

Hence, employing (2.83) and (2.90), the distorted wave $\chi_{i}^{+}$becomes:

$$
\begin{gathered}
\chi_{i}^{+}=\mu_{i}^{-2 i v_{P}} \mathcal{N}^{+}\left(v_{\mathrm{PT}}\right)\left[N^{+}\left(\nu_{\mathrm{P}}\right)\right]^{2} \mathrm{e}^{i \boldsymbol{k}_{i} \cdot \boldsymbol{r}_{i}} \varphi_{i}\left(\boldsymbol{x}_{1}, \boldsymbol{x}_{2}\right)_{1} F_{1}\left(-i \nu_{\mathrm{PT}}, 1, i k_{i} \boldsymbol{r}_{f}+i \boldsymbol{k}_{i} \cdot \boldsymbol{r}_{f}\right) \\
\times{ }_{1} F_{1}\left(i \nu_{\mathrm{P}}, 1, i v s_{1}+i \boldsymbol{v} \cdot \boldsymbol{s}_{1}\right)_{1} F_{1}\left(i \nu_{\mathrm{P}}, 1, i v s_{2}+i \boldsymbol{v} \cdot \boldsymbol{s}_{2}\right) .
\end{gathered}
$$

\subsubsection{Exit channel}

Similarly to (2.83), an inspection of the boundary condition requirement (2.65) in the exit channel suggests a factorized form for $\chi_{f}^{-}$of the type:

$$
\chi_{f}^{-}=\varphi_{f}\left(s_{1}, s_{2}\right) \zeta_{f}^{-}
$$

where $\zeta_{f}^{-}$is a function to be found. Substituting (2.94) into (2.62) and employing (2.40) and $H_{0}$ from (2.15), the following equation is deduced for $\zeta_{f}^{-}$:

$$
\varphi_{f}\left(\Delta E_{f}-H_{0}-V_{f}\right) \zeta_{f}^{-}+\left\{\sum_{k=1}^{2} \frac{1}{a_{k}} \nabla_{s_{k}} \varphi_{f} \cdot \nabla_{s_{k}} \zeta_{f}^{-}+U_{f} \varphi_{f} \zeta_{f}^{-}\right\}=0
$$

where

$$
\Delta E_{f}=E_{f}-\epsilon_{f}^{\mathrm{P}}=\frac{k_{f}^{2}}{2 \mu_{f}} .
$$

Symmetrically, with respect to $U_{i}$ from (2.84), the following choice of the operator potential $U_{f}$ cancels out the terms within the curly brackets from (2.95):

$$
U_{f}=-\sum_{k=1}^{2} \frac{1}{a_{k}} \nabla_{s_{k}} \ln \varphi_{f} \cdot \nabla_{s_{k}}
$$

Here too, the reciprocal $1 / \varphi_{f}$, stemming from $\nabla_{s_{k}} \ln \varphi_{f}=\left(1 / \varphi_{f}\right) \nabla_{s_{k}} \varphi_{f}$, is not a problem even for the nodes of $\varphi_{f}$. This occurs because, by definition, the operator $U_{f}$ applies only to the class of functions that are the product of $\varphi_{f}$ with some other functions. Such is the distorted wave $\chi_{f}^{-}=\varphi_{f} \zeta_{f}^{-}$from (2.94) and, therefore:

$$
\nabla_{s_{k}} \ln \varphi_{f} \cdot \nabla_{s_{k}} \chi_{f}^{-}=\nabla_{s_{k}} \varphi_{f} \cdot \nabla_{s_{k}} \zeta_{f}^{-}
$$


This occurrence implies that $U_{f} \chi_{f}^{-}$is a regular function:

$$
U_{f} \chi_{f}^{-}=-\sum_{k=1}^{2} \frac{1}{a_{k}} \nabla_{s_{k}} \varphi_{f} \cdot \nabla_{s_{k}} \zeta_{f}^{-}
$$

which reduces Eq. (2.95) to

$$
\left(\Delta E_{f}-H_{0}-V_{f}\right) \zeta_{f}^{-}=0 .
$$

In order to proceed with this equation, we employ $H_{0}$ from (2.14). Moreover, in $V_{f}$ from (2.100), the eikonal mass limit for the $R$-dependent potential implies that $Z_{\mathrm{P}} Z_{\mathrm{T}} / R \approx Z_{\mathrm{P}} Z_{\mathrm{T}} / r_{i}$. In this situation, the choice (2.99) for $U_{f}$ makes Eq. (2.100) separable in the independent variables $\left\{\boldsymbol{r}_{i}, \boldsymbol{x}_{1}, \boldsymbol{x}_{2}\right\}$. With such an arrangement, the solution of Eq. (2.100), satisfying the imposed asymptotic behavior (2.65), is found to be:

$$
\begin{aligned}
\zeta_{f}^{-}= & \mu_{f}^{-2 i v_{T}} \mathcal{N}^{-}\left(\nu_{\mathrm{PT}}\right)\left[N^{-}\left(\nu_{\mathrm{T}}\right)\right]^{2} \mathrm{e}^{-i \boldsymbol{k}_{f} \cdot \boldsymbol{r}_{f}}{ }_{1} F_{1}\left(i \nu_{\mathrm{PT}}, 1,-i k_{f} r_{i}-i \boldsymbol{k}_{f} \cdot \boldsymbol{r}_{i}\right) \\
& \times{ }_{1} F_{1}\left(-i \nu_{\mathrm{T}}, 1,-i v x_{1}-i \boldsymbol{v} \cdot \boldsymbol{x}_{1}\right)_{1} F_{1}\left(-i v_{\mathrm{T}}, 1,-i v x_{2}-i \boldsymbol{v} \cdot \boldsymbol{x}_{2}\right) .
\end{aligned}
$$

Therefore, using (2.94) and (2.101), we can write the distorted wave $\chi_{f}^{-}$as:

$$
\begin{aligned}
\chi_{f}^{-}= & \mu_{f}^{-2 i v_{T}} \mathcal{N}^{-}\left(\nu_{\mathrm{PT}}\right)\left[N^{-}\left(\nu_{\mathrm{T}}\right)\right]^{2} \mathrm{e}^{-i \boldsymbol{k}_{f} \cdot \boldsymbol{r}_{f}} \varphi_{f}\left(\boldsymbol{s}_{1}, \boldsymbol{s}_{2}\right)_{1} F_{1}\left(i \nu_{\mathrm{PT}}, 1,-i k_{f} r_{i}-i \boldsymbol{k}_{f} \cdot \boldsymbol{r}_{i}\right) \\
& \times{ }_{1} F_{1}\left(-i v_{\mathrm{T}}, 1,-i v x_{1}-i \boldsymbol{v} \cdot \boldsymbol{x}_{1}\right)_{1} F_{1}\left(-i \nu_{\mathrm{T}}, 1,-i v x_{2}-i \boldsymbol{v} \cdot \boldsymbol{x}_{2}\right) .
\end{aligned}
$$

\subsection{Asymptotic behaviors of distorted waves at large inter-particle distances}

In the entrance channel, for large $s_{1}$ and $s_{2}$, using the asymptotic formula of the confluent hypergeometric function, we have:

$$
\left[N^{+}\left(\nu_{\mathrm{P}}\right)\right]^{2} \prod_{k=1}^{2}{ }_{1} F_{1}\left(i \nu_{\mathrm{P}}, 1, i v s_{k}+i \boldsymbol{v} \cdot \boldsymbol{s}_{k}\right) \underset{s_{1}, s_{2} \rightarrow \infty}{\approx} \prod_{k=1}^{2}\left(v s_{k}+\boldsymbol{v} \cdot \boldsymbol{s}_{k}\right)^{-i \nu_{\mathrm{P}}}
$$

Also the asymptotes of the two Kummer functions for large $x_{1}$ and $x_{2}$ in the exit channel yield:

$$
\left[N^{-}\left(v_{\mathrm{T}}\right)\right]^{2} \prod_{k=1}^{2} 1 F_{1}\left(-i v_{\mathrm{T}}, 1,-i v x_{k}-i \boldsymbol{v} \cdot \boldsymbol{x}_{k}\right) \underset{x_{1}, x_{2} \rightarrow \infty}{\approx} \prod_{k=1}^{2}\left(v x_{k}+\boldsymbol{v} \cdot \boldsymbol{x}_{k}\right)^{i v_{\mathrm{T}}}
$$

In these asymptotic cases, the distorted waves from (2.93) and (2.102) are transformed to: 


$$
\begin{aligned}
\chi_{i}^{+}= & \mu_{i}^{-2 i v_{P}} \mathcal{N}^{+}\left(\nu_{\mathrm{PT}}\right) \mathrm{e}^{i \boldsymbol{k}_{i} \cdot \boldsymbol{r}_{i}} \varphi_{i}\left(\boldsymbol{x}_{1}, \boldsymbol{x}_{2}\right){ }_{1} F_{1}\left(-i \nu_{\mathrm{PT}}, 1, i k_{i} \boldsymbol{r}_{f}+i \boldsymbol{k}_{i} \cdot \boldsymbol{r}_{f}\right) \\
& \times\left(v s_{1}+\boldsymbol{v} \cdot \boldsymbol{s}_{1}\right)^{-i \nu_{\mathrm{P}}}\left(v s_{2}+\boldsymbol{v} \cdot \boldsymbol{s}_{2}\right)^{-i \nu_{\mathrm{P}}}, \\
\chi_{f}^{-}= & \mu_{f}^{-2 i v_{T}} \mathcal{N}^{-}\left(\nu_{\mathrm{PT}}\right) \mathrm{e}^{-i \boldsymbol{k}_{f} \cdot \boldsymbol{r}_{f}} \varphi_{f}\left(\boldsymbol{s}_{1}, \boldsymbol{s}_{2}\right)_{1} F_{1}\left(i \nu_{\mathrm{PT}}, 1,-i k_{f} r_{i}-i \boldsymbol{k}_{f} \cdot \boldsymbol{r}_{i}\right) \\
& \times\left(v x_{1}+\boldsymbol{v} \cdot \boldsymbol{x}_{1}\right)^{i \nu_{\mathrm{T}}}\left(v x_{2}+\boldsymbol{v} \cdot \boldsymbol{x}_{2}\right)^{i \nu_{\mathrm{T}}} .
\end{aligned}
$$

Moreover, in the asymptotic region of large inter-particle distances for the entrance channel, all the three coordinates $s_{1}, s_{2}$ and $R$ are simultaneously large, so that:

$$
\lim _{s_{k} \rightarrow \infty, R \rightarrow \infty} \frac{v R-\boldsymbol{v} \cdot \boldsymbol{R}}{v s_{k}+\boldsymbol{v} \cdot \boldsymbol{s}_{k}}=1 \quad(k=1,2) .
$$

Likewise, all the three coordinates $x_{1}, x_{2}$ and $R$ become simultaneously large in the asymptotic region of the exit channel and, therefore:

$$
\lim _{x_{k} \rightarrow \infty, R \rightarrow \infty} \frac{v R+\boldsymbol{v} \cdot \boldsymbol{R}}{v x_{k}+\boldsymbol{v} \cdot \boldsymbol{x}_{k}}=1 \quad(k=1,2) .
$$

Further, for large inter-nuclear separation $R$, the distances $r_{i, f}$ are also large. In fact, even at any value of $R$, due to heavy masses $M_{\mathrm{P}, \mathrm{T}} \gg 1$, we have $\boldsymbol{r}_{i, f} \approx \pm \boldsymbol{R}$, according to (2.17). This implies:

$$
\begin{aligned}
\lim _{s_{k} \rightarrow \infty, r_{f} \rightarrow \infty} \frac{k_{i} r_{f}+\boldsymbol{k}_{i} \cdot \boldsymbol{r}_{f}}{v s_{k}+\boldsymbol{v} \cdot \boldsymbol{s}_{k}} & =\mu_{i} \quad(k=1,2), \\
\lim _{x_{k} \rightarrow \infty, R \rightarrow \infty} \frac{k_{f} r_{i}+\boldsymbol{k}_{f} \cdot \boldsymbol{r}_{i}}{v x_{k}+\boldsymbol{v} \cdot \boldsymbol{x}_{k}} & =\mu_{f} \quad(k=1,2) .
\end{aligned}
$$

With these asymptotes at hand, the following expressions are obtained:

$$
\begin{array}{ll}
\chi_{i}^{+} s_{1}, \overrightarrow{s_{2}, r_{f}} \rightarrow \infty & \Phi_{i} \mathrm{e}^{i v_{i} \ln \mu(v R-\boldsymbol{v} \cdot \boldsymbol{R})}=\Phi_{i}^{+}, \\
\chi_{f}^{-} x_{1}, \overrightarrow{x_{2}, r_{i}} \rightarrow \infty & \Phi_{f} \mathrm{e}^{-i v_{f} \ln \mu(v R+\boldsymbol{v} \cdot \boldsymbol{R})}=\Phi_{f}^{-},
\end{array}
$$

in agreement with the requirements (2.64) and (2.65). Moreover, as per the derivation, the distorted waves $\chi_{i}^{+}$and $\chi_{f}^{-}$are automatically connected to the distorting potentials $U_{i}$ and $U_{f}$ by way of Eqs. (2.69) and (2.70). As such, both prerequisites from (2.82) are fulfilled. Therefore, $\chi_{i}^{+}$and $\chi_{f}^{-}$from (2.93) and (2.102) satisfy the correct boundary conditions, as per the requests in (2.82).

Overall, it is important to emphasize that only in the asymptotic regions of the entrance channel, is it permitted to replace the terms $v s_{k}+\boldsymbol{v} \cdot \boldsymbol{s}_{k}(k=1,2)$ by $v R-\boldsymbol{v} \cdot \boldsymbol{R}$. A similar replacement of $v x_{k}+\boldsymbol{v} \cdot \boldsymbol{x}_{k}(k=1,2)$ by $v R+\boldsymbol{v} \cdot \boldsymbol{R}$ is allowed solely at the asymptotic distances in the exit channel:

$$
\begin{aligned}
& \Phi_{i} \mathrm{e}^{-i \nu_{\mathrm{P}} \ln \left(v s_{1}+\boldsymbol{v} \cdot \boldsymbol{s}_{1}\right)-i \nu_{\mathrm{P}} \ln \left(v s_{2}+\boldsymbol{v} \cdot \boldsymbol{s}_{2}\right)} \underset{s_{1}, s_{2}, R \rightarrow \infty}{\longrightarrow} \Phi_{i} \mathrm{e}^{-2 i \nu_{\mathrm{P}} \ln (v R-\boldsymbol{v} \cdot \boldsymbol{R})}, \\
& \Phi_{f} \mathrm{e}^{i \nu_{\mathrm{T}} \ln \left(v x_{1}+\boldsymbol{v} \cdot \boldsymbol{x}_{1}\right)+i v_{\mathrm{T}} \ln \left(v x_{2}+\boldsymbol{v} \cdot \boldsymbol{x}_{2}\right)} \underset{x_{1}, x_{2}, R \rightarrow \infty}{\longrightarrow} \Phi_{f} \mathrm{e}^{2 i \nu_{\mathrm{T}} \ln (v R+\boldsymbol{v} \cdot \boldsymbol{R})} .
\end{aligned}
$$


However, the transition amplitudes are the integrals over every inter-particle distance, i.e. these integrals do not cover only the asymptotic separations. Therefore, throughout the transition amplitudes, the wave functions $\Phi_{i} \prod_{k=1}^{2}\left(v s_{k}+v \cdot s_{k}\right)^{-i \nu_{\mathrm{P}}}$ and $\Phi_{i}(v R-$ $\boldsymbol{v} \cdot \boldsymbol{R})^{-2 i \nu_{\mathrm{P}}}$ from the entrance channel are not equivalent. Likewise, throughout the transition amplitudes, the wave functions $\Phi_{f} \prod_{k=1}^{2}\left(v x_{k}+\boldsymbol{v} \cdot \boldsymbol{x}_{k}\right)^{i \nu_{\mathrm{T}}}$ and $\Phi_{f}(v R+$ $\boldsymbol{v} \cdot \boldsymbol{R})^{2 i v_{\mathrm{T}}}$ from the exit channel are not equivalent either. This means that the use of $\Phi_{i} \prod_{k=1}^{2}\left(v s_{k}+\boldsymbol{v} \cdot \boldsymbol{s}_{k}\right)^{-i \nu_{\mathrm{P}}}$ and $\Phi_{i}(v R-\boldsymbol{v} \cdot \boldsymbol{R})^{-2 i \nu_{\mathrm{P}}}$ in the transition amplitudes would give two different methods, e.g. the CDW-EIS-4B and the post BDW-4B method, respectively. Similarly, employing $\Phi_{f} \prod_{k=1}^{2}\left(v x_{k}+\boldsymbol{v} \cdot \boldsymbol{x}_{k}\right)^{i v_{\mathrm{T}}}$ and $\Phi_{f}(v \boldsymbol{R}+\boldsymbol{v} \cdot \boldsymbol{R})^{2 i v_{\mathrm{T}}}$ in the transition amplitudes would yield another pair of differing methods, e.g. the CDW-EFS-4B and the prior BDW-4B method, respectively.

\subsection{Different choices of the distorting potentials and distorted waves}

Second-order theories are the formalisms that include the intermediate ionization channels for electronic degrees of freedom through the Coulomb wave functions of the electrons centered on one or both nuclei. Symmetric second-order theories, such as the CDW-4B method, have the electronic Coulomb wave functions centered on both nuclei with two such functions in each channel (entrance and exit). Asymmetric second-order theories, e.g. the BCIS-4B and BDW-4B methods, possess two electronic Coulomb wave functions in total, both centered on one nucleus in one channel alone (entrance or exit) for the given transition amplitude. There is also another pair of asymmetric second-order theories, e.g. the CDW-EIS-4B and CDW-EFS-4B methods, that use four electronic distorting functions, such as two full Coulomb waves in one channel (exit/entrance) and two Coulomb logarithmic phases in the complementary channel (entrance/exit), respectively. First-order theories are the formalisms that do not include any intermediate ionization channels for the two captured electrons. Symmetric first-order theories, e.g. the CB1-4B method, include one Coulomb wave function (or equivalently, its logarithmic phase factor) per channel (entrance or exit) for the relative motion of heavy nuclei. Both the BCIS-4B and BDW-4B method belong to the class of hybrid theories that treat one channel by the CDW-4B method and the other by the CB1-4B method. The CDW-EIS/EFS-4B are also from the category of hybrid theories since they coincide with the CDW-4B in one channel, whereas the electronic and nuclear distorting functions in the other channel are eikonalized.

\subsubsection{Symmetric second-order theories: four-body continuum distorted wave method, CDW-4B}

For the prior and post transition amplitudes (2.80) and (2.81), we make the first symmetric choices of the initial and final pairs $\left\{U_{i, f}, \chi_{i, f}^{ \pm}\right\}$in the entrance and exit channels according to the following selection, which defines the four-body continuum distorted wave method, CDW-4B [30,31,43-45]: 


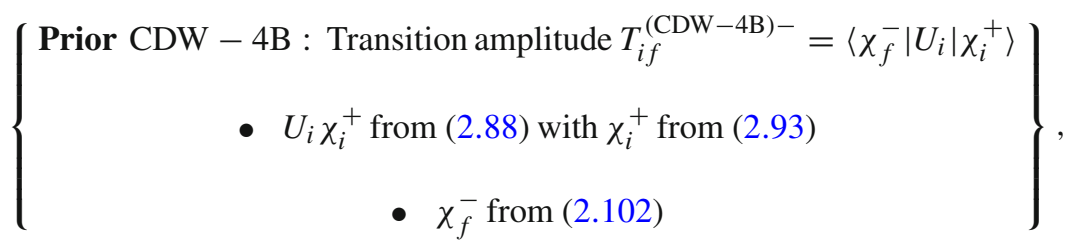

$\left\{\begin{array}{c}\text { Post CDW - 4B : Transition amplitude } T_{i f}^{(\mathrm{CDW}-4 \mathrm{~B})+}=\left\langle\chi_{f}^{-}\left|U_{f}^{\dagger}\right| \chi_{i}^{+}\right\rangle \\ \bullet \chi_{i}^{+} \text {from }(2.93) \\ \bullet U_{f} \chi_{f}^{-} \text {from (2.99) with } \chi_{f}^{-} \text {from (2.102) }\end{array}\right\}$.

The corresponding explicit prior and post transition amplitudes $T_{i f}^{(\mathrm{CDW}-4 \mathrm{~B}) \mp}$ read as:

$$
\begin{aligned}
T_{i f}^{(\mathrm{CDW}-4 \mathrm{~B})-}=- & N^{2} C_{i f} \iiint \mathrm{d} \boldsymbol{x}_{1} \mathrm{~d} \boldsymbol{x}_{2} \mathrm{~d} \boldsymbol{r}_{i} \\
& \times \mathrm{e}^{i \boldsymbol{k}_{i} \cdot \boldsymbol{r}_{i}+i \boldsymbol{k}_{f} \cdot \boldsymbol{r}_{f}} \mathcal{L}_{i f}^{(\mathrm{CDW}-4 \mathrm{~B})-} \mathcal{B}_{i f}^{(\mathrm{CDW}-4 \mathrm{~B})-}, \\
T_{i f}^{(\mathrm{CDW}-4 \mathrm{~B})+}=- & N^{2} C_{i f} \iiint \mathrm{d} \boldsymbol{s}_{1} \mathrm{~d} \boldsymbol{s}_{2} \mathrm{~d} \boldsymbol{r}_{f} \\
& \times \mathrm{e}^{i \boldsymbol{k}_{i} \cdot \boldsymbol{r}_{i}+i \boldsymbol{k}_{f} \cdot \boldsymbol{r}_{f}} \mathcal{L}_{i f}^{(\mathrm{CDW}-4 \mathrm{~B})+} \mathcal{B}_{i f}^{(\mathrm{CDW}-4 \mathrm{~B})+},
\end{aligned}
$$

where

$$
C_{i f}=\mu_{i}^{-2 i \nu_{\mathrm{P}}} \mu_{f}^{-2 i \nu_{\mathrm{T}}}, \quad N=N^{+}\left(v_{\mathrm{P}}\right) N^{-*}\left(\nu_{\mathrm{T}}\right)
$$

and

$$
\begin{aligned}
& \mathcal{B}_{i f}^{(\mathrm{CDW}-4 \mathrm{~B})-}=\varphi_{f}^{*}\left(\boldsymbol{s}_{1}, \boldsymbol{s}_{2}\right){ }_{1} F_{1}\left(i v_{\mathrm{T}}, 1, i v x_{1}+i \boldsymbol{v} \cdot \boldsymbol{x}_{1}\right)_{1} F_{1}\left(i v_{\mathrm{T}}, 1, i v x_{2}+i \boldsymbol{v} \cdot \boldsymbol{x}_{2}\right) \\
& \times\left\{{ }_{1} F_{1}\left(i v_{\mathrm{P}}, 1, i v s_{2}+i \boldsymbol{v} \cdot \boldsymbol{s}_{2}\right) \nabla_{x_{1}} \varphi_{i}\left(\boldsymbol{x}_{1}, \boldsymbol{x}_{2}\right) \cdot \nabla_{s_{1} 1} F_{1}\left(i v_{\mathrm{P}}, 1, i v s_{1}+i \boldsymbol{v} \cdot \boldsymbol{s}_{1}\right)\right. \\
& \left.+{ }_{1} F_{1}\left(i v_{\mathrm{P}}, 1, i v s_{1}+i \boldsymbol{v} \cdot \boldsymbol{s}_{1}\right) \nabla_{x_{2}} \varphi_{i}\left(\boldsymbol{x}_{1}, \boldsymbol{x}_{2}\right) \cdot \nabla_{s_{2} 1} F_{1}\left(i v_{\mathrm{P}}, 1, i v s_{2}+i \boldsymbol{v} \cdot \boldsymbol{s}_{2}\right)\right\} \text {, } \\
& \mathcal{B}_{i f}^{(\mathrm{CDW}-4 \mathrm{~B})+}=\varphi_{i}\left(\boldsymbol{x}_{1}, \boldsymbol{x}_{2}\right)_{1} F_{1}\left(i \nu_{\mathrm{P}}, 1, i v s_{1}+i \boldsymbol{v} \cdot \boldsymbol{s}_{1}\right)_{1} F_{1}\left(i \nu_{\mathrm{P}}, 1, i v s_{2}+i \boldsymbol{v} \cdot \boldsymbol{s}_{2}\right) \\
& \times\left\{{ }_{1} F_{1}\left(i v_{\mathrm{T}}, 1, i v x_{2}+i \boldsymbol{v} \cdot \boldsymbol{x}_{2}\right) \nabla_{s_{1}} \varphi_{f}^{*}\left(\boldsymbol{s}_{1}, \boldsymbol{s}_{2}\right) \cdot \nabla_{x_{1} 1} F_{1}\left(i v_{\mathrm{T}}, 1, i v x_{1}+i \boldsymbol{v} \cdot \boldsymbol{x}_{1}\right)\right. \\
& \left.+{ }_{1} F_{1}\left(i v_{\mathrm{T}}, 1, i v x_{1}+i \boldsymbol{v} \cdot \boldsymbol{x}_{1}\right) \nabla_{s_{2}} \varphi_{f}^{*}\left(\boldsymbol{s}_{1}, \boldsymbol{s}_{2}\right) \cdot \nabla_{x_{2} 1} F_{1}\left(i v_{\mathrm{T}}, 1, i v x_{2}+i \boldsymbol{v} \cdot \boldsymbol{x}_{2}\right)\right\} \text {, } \\
& \mathcal{L}_{i f}^{(\mathrm{CDW}-4 \mathrm{~B})-}=\mathcal{L}_{i f}^{(\mathrm{CDW}-4 \mathrm{~B})+} \\
& =\mathcal{N}\left(v_{\mathrm{PT}}\right)_{1} F_{1}\left(-i \nu_{\mathrm{PT}}, 1, i k_{i} \boldsymbol{r}_{f}+i \boldsymbol{k}_{i} \cdot \boldsymbol{r}_{f}\right){ }_{1} F_{1}\left(-i v_{\mathrm{PT}}, 1, i k_{f} r_{i}+i \boldsymbol{k}_{f} \cdot \boldsymbol{r}_{i}\right) \text {, }
\end{aligned}
$$


with $\mathcal{N}\left(v_{\mathrm{PT}}\right)=\mathcal{N}^{+}\left(\nu_{\mathrm{PT}}\right) \mathcal{N}^{-*}\left(\nu_{\mathrm{PT}}\right)=\left[\mathcal{N}^{+}\left(\nu_{\mathrm{PT}}\right)\right]^{2}$ where $\mathcal{N}^{-*}\left(v_{\mathrm{PT}}\right)=\mathcal{N}^{+}\left(\nu_{\mathrm{PT}}\right)$.

- Independence of the eikonal total cross sections on the inter-nuclear potential

In the eikonal formalism (2.17), the two confluent hypergeometric functions ${ }_{1} F_{1}$ from (2.122) can be replaced by their Coulomb logarithmic phases, and this leads to:

$$
\begin{aligned}
\mathcal{L}_{i f}^{(\mathrm{CDW}-4 \mathrm{~B}) \mp} \underset{M_{\mathrm{P}, \mathrm{T}} \gg 1}{\underset{M_{\mathrm{P}, \mathrm{T}} \gg 1}{\approx}}\left(k_{i} r_{f}+\boldsymbol{k}_{i} \cdot \boldsymbol{r}_{f}\right)^{i \nu_{\mathrm{PT}}}\left(k_{f} r_{i}+\boldsymbol{k}_{f} \cdot \boldsymbol{r}_{i}\right)^{i \nu_{\mathrm{PT}}} \\
=\left[\mu_{f}[(v R-\boldsymbol{v} \cdot \boldsymbol{R})(v R+\boldsymbol{v} \cdot \boldsymbol{R})]\right\}^{i \nu_{\mathrm{PT}}} \\
=\left[\mu_{i} \mu_{f} v^{2}\left(R^{2}-Z^{2}\right)\right]^{i \nu_{\mathrm{PT}}}=\left(\mu_{i} \mu_{f} v^{2} \rho^{2}\right)^{i \nu_{\mathrm{PT}}} \underset{M_{\mathrm{P}, \mathrm{T}} \gg 1}{\approx}(\mu v \rho)^{2 i \nu_{\mathrm{PT}}},
\end{aligned}
$$

so that

$$
\therefore \quad \mathcal{L}_{i f}^{(\mathrm{CDW}-4 \mathrm{~B}) \mp} \underset{M_{\mathrm{P}, \mathrm{T}} \gg 1}{\approx} \quad(\mu v \rho)^{2 i \nu_{\mathrm{PT}}}
$$

where $\rho=|\rho|$ with $\rho$ being the XOY-component of $\boldsymbol{R}$ from (2.6). The remaining phase factor $(\mu v \rho)^{2 i \nu_{\mathrm{PT}}}$ in (2.123) stems directly from the inter-nuclear potential $V_{\mathrm{PT}}=Z_{\mathrm{P}} Z_{\mathrm{T}} / R$. In other words, this latter phase is the only trace left from $V_{\mathrm{PT}}$ in the eikonal versions of the transition amplitudes $T_{i f}^{(\mathrm{CDW}-4 \mathrm{~B}) \mp}$.

Employing (2.4) and (2.6), the exponential function $\mathrm{e}^{i \boldsymbol{k}_{i} \cdot \boldsymbol{r}_{i}+i \boldsymbol{k}_{f} \cdot \boldsymbol{r}_{f}}$ from (2.117) and (2.118) can be written as:

$$
\left.\begin{array}{c}
\mathrm{e}^{i \boldsymbol{k}_{i} \cdot \boldsymbol{r}_{i}+i \boldsymbol{k}_{f} \cdot \boldsymbol{r}_{f}}=\mathcal{D} \\
=\mathrm{e}^{2 i \boldsymbol{\beta} \cdot \boldsymbol{R}-i \boldsymbol{v} \cdot\left(\boldsymbol{s}_{1}+\boldsymbol{s}_{2}\right)}=\mathrm{e}^{2 i \boldsymbol{\alpha} \cdot \boldsymbol{R}-i \boldsymbol{v} \cdot\left(\boldsymbol{x}_{1}+\boldsymbol{x}_{2}\right)} \\
=\mathrm{e}^{-i \boldsymbol{\eta} \cdot \boldsymbol{\rho}-i v^{-} Z-i \boldsymbol{v} \cdot\left(\boldsymbol{s}_{1}+\boldsymbol{s}_{2}\right)}=\mathrm{e}^{i \boldsymbol{\eta} \cdot \boldsymbol{\rho}-i v^{+} Z-i \boldsymbol{v} \cdot\left(\boldsymbol{x}_{1}+\boldsymbol{x}_{2}\right)}
\end{array}\right\},
$$

where

$$
\mathcal{D}=\mathrm{e}^{2 i \boldsymbol{\alpha} \cdot\left(\boldsymbol{s}_{1}+\boldsymbol{s}_{2}\right)+2 i \boldsymbol{\beta} \cdot\left(\boldsymbol{x}_{1}+\boldsymbol{x}_{2}\right)} .
$$

Thus, using (2.123), the transition amplitudes (2.117) and (2.118) become eikonalized in the relative motion of heavy nuclei:

$$
\begin{gathered}
T_{i f}^{(\mathrm{CDW}-4 \mathrm{~B})-}{ }_{(\boldsymbol{\eta})}=-N^{2} C_{i f}^{\prime} \iiint \mathrm{d} \boldsymbol{x}_{1} \mathrm{~d} \boldsymbol{x}_{2} \mathrm{~d} \boldsymbol{R} \rho^{2 i \nu_{\mathrm{PT}}} \mathcal{D} \mathcal{B}_{i f}^{(\mathrm{CDW}-4 \mathrm{~B})-}, \\
T_{i f}^{(\mathrm{CDW}-4 \mathrm{~B})+}{ }_{(\boldsymbol{\eta})}=-N^{2} C_{i f}^{\prime} \iiint \mathrm{d} \boldsymbol{s}_{1} \mathrm{~d} \boldsymbol{s}_{2} \mathrm{~d} \boldsymbol{R} \rho^{2 i \nu_{\mathrm{PT}}} \mathcal{D} \mathcal{B}_{i f}^{(\mathrm{CDW}-4 \mathrm{~B})+},
\end{gathered}
$$

where

$$
C_{i f}^{\prime}=C_{i f}(\mu v)^{2 i \nu_{\mathrm{PT}}}, \quad C_{i f}^{\prime} \underset{M_{\mathrm{P}, \mathrm{T}} \gg 1}{\approx} v^{2 i \nu_{\mathrm{PT}}} \mu^{2 i\left(\nu_{\mathrm{PT}}-\nu_{\mathrm{P}}-\nu_{\mathrm{T}}\right)}
$$

Hereafter, whenever using (2.124) in the transition amplitudes $T_{i f}^{(\mathrm{CDW}-4 \mathrm{~B}) \mp}$ from the general expressions (2.117) and (2.118), the dependence on $\eta$ will explicitly 
be written as $T_{i f}^{(\mathrm{CDW}-4 \mathrm{~B}) \mp}(\eta)$. With this setting, in the expressions for the total cross sections, the starting integration over the solid angle $\Omega_{\mathrm{P}} \equiv\left\{\theta_{\mathrm{P}}, \phi_{\mathrm{P}}\right\}=$ \{scattering angle, azimuthal angle $\}$ around the projectile $\mathrm{P}$ is mapped to the integration over $\eta$, so that:

$$
Q_{i f}^{(\mathrm{CDW}-4 \mathrm{~B}) \mp}\left(a_{0}^{2}\right)=\left.\int \mathrm{d} \boldsymbol{\frac { T _ { i f } ^ { ( \mathrm { CDW } - 4 \mathrm { B } ) \mp } ( \boldsymbol { \eta } ) } { 2 \pi v }}\right|^{2} .
$$

In order to see the net effect of $\rho^{2 i \nu_{\mathrm{PT}}}$ onto the total cross sections, we first pass from one to another set of the volume elements in (2.126) and (2.127) via $\mathrm{d} \boldsymbol{x}_{1} \mathrm{~d} \boldsymbol{x}_{2} \mathrm{~d} \boldsymbol{r}_{i}=$ $J^{-} \mathrm{d} \boldsymbol{x}_{1} \mathrm{~d} \boldsymbol{x}_{2} \mathrm{~d} \boldsymbol{R}$ and $\mathrm{d} \boldsymbol{s}_{1} \mathrm{~d} \boldsymbol{s}_{2} \mathrm{~d} \boldsymbol{r}_{f}=J^{+} \mathrm{d} \boldsymbol{s}_{1} \mathrm{~d} \boldsymbol{s}_{2} \mathrm{~d} \boldsymbol{R}$, respectively, where the Jacobians $J^{\mp}$ are checked to be equal to unity. Subsequently, we use $\mathrm{d} \boldsymbol{R}=\mathrm{d} \rho \mathrm{d} Z$ from (2.6) in Eqs. (2.117) and (2.118) for $T_{i f}^{(\mathrm{CDW}-4 \mathrm{~B})-}$ and $T_{i f}^{(\mathrm{CDW}-4 \mathrm{~B})+}$. Then before performing the integrals over $\left\{\boldsymbol{x}_{1}, \boldsymbol{x}_{2}, Z\right\}$ in (2.126) and over $\left\{\boldsymbol{s}_{1}, \boldsymbol{s}_{2}, Z\right\}$ in (2.127), we first integrate the functions $\left|T_{i f}^{(\mathrm{CDW}-4 \mathrm{~B}) \mp}(\eta)\right|^{2}=T_{i f}^{(\mathrm{CDW}-4 \mathrm{~B}) \mp}(\eta)\left\{T_{i f}^{(\mathrm{CDW}-4 \mathrm{~B}) \mp}(\eta)\right\}^{*}$ over $\eta$ in $Q_{i f}^{(\mathrm{CDW}-4 \mathrm{~B}) \mp}$ from (2.129). This latter integral over $\boldsymbol{\eta}$ yields the Dirac deltafunction $\delta\left(\rho-\rho^{\prime}\right)$ which, in turn, removes the phase $\rho^{i \nu_{\mathrm{PT}}}$ from eikonal total cross sections $Q_{i f}^{(\mathrm{CDW}-4 \mathrm{~B}) \mp}$. In fact, two pairs of such phases $\rho^{i \nu_{\mathrm{PT}}}$ and $\left(\rho^{\prime}\right)^{-i \nu_{\mathrm{PT}}}$, one from $T_{i f}^{(\mathrm{CDW}-4 \mathrm{~B}) \mp}$ and the other from $\left\{T_{i f}^{(\mathrm{CDW}-4 \mathrm{~B}) \mp}\right\}^{*}$, appear as $\rho^{i \nu_{\mathrm{PT}}}\left(\rho^{\prime}\right)^{-i \nu_{\mathrm{PT}}} \delta\left(\rho-\rho^{\prime}\right)$ where they are canceled by the $\delta$-function in the remaining integral over $\rho$. Overall, this procedure shows that the inter-nuclear potential $V_{\mathrm{PT}}$ has no influence whatsoever on the eikonal total cross sections $Q_{i f}^{(\mathrm{CDW}-4 \mathrm{~B}) \mp}$.

All told, the eikonal transition amplitudes $T_{i f}^{(\mathrm{CDW}-4 \mathrm{~B}) \mp}$ from (2.117) and (2.118) now become:

$$
T_{i f}^{(\mathrm{CDW}-4 \mathrm{~B}) \mp}(\boldsymbol{\eta})=\int \mathrm{d} \boldsymbol{\rho} \mathrm{e}^{\mp i \boldsymbol{\eta} \cdot \boldsymbol{\rho}} \rho^{2 i \nu_{\mathrm{PT}}} \mathcal{A}_{i f}^{(\mathrm{CDW}-4 \mathrm{~B}) \mp}(\boldsymbol{\rho}) .
$$

Here, $\mathcal{A}_{i f}^{(\mathrm{CDW}-4 \mathrm{~B}) \mp}(\boldsymbol{\rho})$ denote the mentioned integrals over $\left\{\boldsymbol{x}_{1}, \boldsymbol{x}_{2}, Z\right\}$ within $T_{i f}^{(\mathrm{CDW}-4 \mathrm{~B})-}(\boldsymbol{\eta})$ and over $\left\{\boldsymbol{s}_{1}, \boldsymbol{s}_{2}, Z\right\}$ in $T_{i f}^{(\mathrm{CDW}-4 \mathrm{~B})+}(\boldsymbol{\eta})$ from (2.126) and (2.127), respectively:

$$
\begin{aligned}
& \mathcal{A}_{i f}^{(\mathrm{CDW}-4 \mathrm{~B})-}(\boldsymbol{\rho})=-N^{2} C_{i f}^{\prime} \iiint \mathrm{d} \boldsymbol{x}_{1} \mathrm{~d} \boldsymbol{x}_{2} \mathrm{~d} Z \mathrm{e}^{-i v^{-} Z-i \boldsymbol{v} \cdot\left(\boldsymbol{s}_{1}+\boldsymbol{s}_{2}\right)} \mathcal{B}_{i f}^{(\mathrm{CDW}-4 \mathrm{~B})-} \\
& \mathcal{A}_{i f}^{(\mathrm{CDW}-4 \mathrm{~B})-}(\boldsymbol{\rho})=-N^{2} C_{i f}^{\prime} \iiint \mathrm{d} \boldsymbol{s}_{1} \mathrm{~d} \boldsymbol{s}_{2} \mathrm{~d} Z \mathrm{e}^{-i v^{+} Z-i \boldsymbol{v} \cdot\left(\boldsymbol{x}_{1}+\boldsymbol{x}_{2}\right)} \mathcal{B}_{i f}^{(\mathrm{CDW}-4 \mathrm{~B})+}
\end{aligned}
$$


Then the associated total cross sections $Q_{i f}^{(\mathrm{CDW}-4 \mathrm{~B}) \mp}$ become:

$$
\begin{aligned}
& Q_{i f}^{(\mathrm{CDW}-4 \mathrm{~B}) \mp}\left(a_{0}^{2}\right) \\
& =\frac{1}{(2 \pi v)^{2}} \int \mathrm{d} \eta T_{i f}^{(\mathrm{CDW}-4 \mathrm{~B}) \mp}(\boldsymbol{\eta})\left\{T_{i f}^{(\mathrm{CDW}-4 \mathrm{~B} \mp}(\boldsymbol{\eta})\right\}^{*} \\
& =\frac{1}{(2 \pi v)^{2}} \iint \mathrm{d} \rho \mathrm{d} \rho^{\prime} \rho^{i \nu_{\mathrm{PT}}}\left(\rho^{\prime}\right)^{-i \nu_{\mathrm{PT}}} \mathcal{A}_{i f}^{(\mathrm{CDW}-4 \mathrm{~B}) \mp}(\rho)\left\{\mathcal{A}_{i f}^{(\mathrm{CDW}-4 \mathrm{~B}) \mp}\left(\rho^{\prime}\right)\right\}^{*} \\
& \times \int \mathrm{d} \eta \mathrm{e}^{\mp i \eta \cdot\left(\rho^{\prime}-\rho\right)} \\
& =\frac{1}{v^{2}} \iint \mathrm{d} \rho \mathrm{d} \rho^{\prime} \mathcal{A}_{i f}^{(\mathrm{CDW}-4 \mathrm{~B}) \mp}(\rho)\left\{\mathcal{A}_{i f}^{(\mathrm{CDW}-4 \mathrm{~B}) \mp}\left(\rho^{\prime}\right)\right\}^{*} \\
& \times\left\{\rho^{i \nu_{\mathrm{PT}}}\left(\rho^{\prime}\right)^{-i \nu_{\mathrm{PT}}} \delta\left(\boldsymbol{\rho}^{\prime}-\boldsymbol{\rho}\right)\right\} \\
& =\frac{1}{v^{2}} \int \mathrm{d} \rho\left|\mathcal{A}_{i f}^{(\mathrm{CDW}-4 \mathrm{~B}) \mp}(\rho)\right|^{2}, \quad \delta\left(\rho^{\prime}-\rho\right)=\delta\left(\rho-\rho^{\prime}\right), \\
& \therefore \quad Q_{i f}^{(\mathrm{CDW}-4 \mathrm{~B}) \mp}\left(a_{0}^{2}\right)=\frac{1}{v^{2}} \int \mathrm{d} \rho\left|\mathcal{A}_{i f}^{(\mathrm{CDW}-4 \mathrm{~B}) \mp}(\boldsymbol{\rho})\right|^{2} .
\end{aligned}
$$

To compare the outcome (2.133) with the starting expression (2.129), we work backward by recuperating the integration over $\eta$ through the re-introduction of the $\delta$-function:

$$
\begin{aligned}
& Q_{i f}^{(\mathrm{CDW}-4 \mathrm{~B}) \mp}\left(a_{0}^{2}\right)=\frac{1}{v^{2}} \int \mathrm{d} \rho\left|\mathcal{A}_{i f}^{(\mathrm{CDW}-4 \mathrm{~B}) \mp}(\boldsymbol{\eta})\right|^{2} \\
& =\frac{1}{v^{2}} \iint \mathrm{d} \rho \mathrm{d} \rho^{\prime} \mathcal{A}_{i f}^{(\mathrm{CDW}-4 \mathrm{~B}) \mp}(\rho)\left\{\mathcal{A}_{i f}^{(\mathrm{CDW}-4 \mathrm{~B}) \mp}\left(\boldsymbol{\rho}^{\prime}\right)\right\}^{*} \delta\left(\rho-\rho^{\prime}\right) \\
& =\frac{1}{(2 \pi v)^{2}} \iint \mathrm{d} \rho \mathrm{d} \rho^{\prime} \mathcal{A}_{i f}^{(\mathrm{CDW}-4 \mathrm{~B}) \mp}(\boldsymbol{\rho})\left\{\mathcal{A}_{i f}^{(\mathrm{CDW}-4 \mathrm{~B}) \mp}\left(\boldsymbol{\rho}^{\prime}\right)\right\}^{*} \int \mathrm{d} \boldsymbol{\eta} \mathrm{e}^{\mp i \eta \cdot\left(\boldsymbol{\rho}-\boldsymbol{\rho}^{\prime}\right)} \\
& =\frac{1}{(2 \pi v)^{2}} \int \mathrm{d} \eta\left\{\int \mathrm{d} \rho \mathrm{e}^{\mp i \eta \cdot \rho} \mathcal{A}_{i f}^{(\mathrm{CDW}-4 \mathrm{~B}) \mp}(\boldsymbol{\rho})\right\}\left\{\int \mathrm{d} \boldsymbol{\rho}^{\prime} \mathrm{e}^{\mp i \boldsymbol{\eta} \cdot \boldsymbol{\rho}^{\prime}} \mathcal{A}_{i f}^{(\mathrm{CDW}-4 \mathrm{~B}) \mp}\left(\boldsymbol{\rho}^{\prime}\right)\right\}^{*} \\
& =\frac{1}{(2 \pi v)^{2}} \int \mathrm{d} \eta\left|R_{i f}^{(\mathrm{CDW}-4 \mathrm{~B}) \mp}(\eta)\right|^{2}, \\
& \therefore \quad Q_{i f}^{(\mathrm{CDW}-4 \mathrm{~B}) \mp}\left(a_{0}^{2}\right)=\int \mathrm{d} \eta\left|\frac{R_{i f}^{(\mathrm{CDW}-4 \mathrm{~B}) \mp}(\eta)}{2 \pi v}\right|^{2},
\end{aligned}
$$

where

$$
R_{i f}^{(\mathrm{CDW}-4 \mathrm{~B}) \mp}(\boldsymbol{\eta})=\int \mathrm{d} \rho \mathrm{e}^{\mp i \eta \cdot \rho} \mathcal{A}_{i f}^{(\mathrm{CDW}-4 \mathrm{~B}) \mp}(\boldsymbol{\rho}) .
$$


From here, the explicit expressions for the matrix elements $R_{i f}^{(\mathrm{CDW}-4 \mathrm{~B}) \mp}(\eta)$ are written as:

$$
\begin{gathered}
R_{i f}^{(\mathrm{CDW}-4 \mathrm{~B})-}(\boldsymbol{\eta})=-N^{2} C_{i f}^{\prime} \iiint \mathrm{d} \boldsymbol{x}_{1} \mathrm{~d} \boldsymbol{x}_{2} \mathrm{~d} \boldsymbol{R} \mathcal{D} \mathcal{B}_{i f}^{(\mathrm{CDW}-4 \mathrm{~B})-} \\
R_{i f}^{(\mathrm{CDW}-4 \mathrm{~B})+}{ }_{(\boldsymbol{\eta})}=-N^{2} C_{i f}^{\prime} \iiint \mathrm{d} \boldsymbol{s}_{1} \mathrm{~d} \boldsymbol{s}_{2} \mathrm{~d} \boldsymbol{R} \mathcal{D} \mathcal{B}_{i f}^{(\mathrm{CDW}-4 \mathrm{~B})+}
\end{gathered}
$$

This shows, by reference to the pairs of Eqs. $\{(2.126),(2.127)\}$ and $\{(2.136),(2.137)\}$, that the only difference between $R_{i f}^{(\mathrm{CDW}-4 \mathrm{~B}) \mp}(\eta)$ and $T_{i f}^{(\mathrm{CDW}-4 \mathrm{~B}) \mp}(\boldsymbol{\eta})$ is in the former matrix elements do not contain the inter-nuclear phase $\rho^{2 i \nu_{\mathrm{PT}}}$ :

$$
R_{i f}^{(\mathrm{CDW}-4 \mathrm{~B}) \mp}(\boldsymbol{\eta})=\left\{T_{i f}^{(\mathrm{CDW}-4 \mathrm{~B}) \mp}(\boldsymbol{\eta})\right\}_{\nu_{\mathrm{PT}}=0, \text { i.e. } V_{\mathrm{PT}}=0},
$$

and consequently

$$
\left\{Q_{i f}^{(\mathrm{CDW}-4 \mathrm{~B}) \mp}\right\}_{\text {with } V_{\mathrm{PT}}}=\left\{Q_{i f}^{(\mathrm{CDW}-4 \mathrm{~B}) \mp}\right\}_{\text {without } V_{\mathrm{PT}}}
$$

Therefore, the eikonal total cross sections $Q_{i f}^{(\mathrm{CDW}-4 \mathrm{~B}) \mp}$ for double charge exchange in four-body collisions via process (2.1) are independent of the inter-nuclear potential $V_{\mathrm{PT}}=Z_{\mathrm{P}} Z_{\mathrm{T}} / R$.

The outlined procedure amounts to implicitly using the Fourier integral transform of the functions with the variables $\boldsymbol{\eta}$ and $\rho$. This is seen from (2.135) where $R_{i f}^{(\mathrm{CDW}-4 \mathrm{~B}) \mp}(\boldsymbol{\eta})$ are the Fourier transforms of $\mathcal{A}_{i f}^{(\mathrm{CDW}-4 \mathrm{~B}) \mp}(\boldsymbol{\rho})$. Likewise, it is seen from (2.130) that the transition amplitudes $T_{i f}^{(\mathrm{CDW}-4 \mathrm{~B}) \mp}(\eta)$ are the Fourier transforms of $\rho^{2 i \nu_{\mathrm{PT}}} \mathcal{A}_{i f}^{(\mathrm{CDW}-4 \mathrm{~B}) \mp}(\rho)$. In fact, the expressions from (2.133) and (2.134) are the Parseval relations that yield the same total cross sections $Q_{i f}^{(\mathrm{CDW}-4 \mathrm{~B}) \mp}$ irrespective of whether computations are carried out in the $\eta$ or the $\rho$ domain of the Fourier transforms:

$$
\begin{aligned}
Q_{i f}^{(\mathrm{CDW}-4 \mathrm{~B}) \mp} & =\frac{1}{(2 \pi v)^{2}} \int \mathrm{d} \eta\left|R_{i f}^{(\mathrm{CDW}-4 \mathrm{~B}) \mp}(\eta)\right|^{2} \\
& =\frac{1}{v^{2}} \int \mathrm{d} \rho\left|\mathcal{A}_{i f}^{(\mathrm{CDW}-4 \mathrm{~B}) \mp}(\rho)\right|^{2}
\end{aligned}
$$

Thus far, the entire exposition was a fully quantum-mechanical treatment for both electrons and nuclei. In such a presentation, treatment of the motion of nuclei does not necessarily need to resort to the eikonal formalism. However, when the eikonal hypothesis is used, as in the present analysis, with the full Coulomb waves replaced by their logarithmic phases for the relative motion of nuclei, the obtained expressions for the transition amplitudes in (2.130) can be interpreted as if they were derived from the four-body impact parameter formulation of the CDW-4B method. Namely, when the latter formalism is adopted from the onset (along the lines of e.g. the BCIS-4B 
method [32]), the nuclear motion is treated classically by a rectilinear trajectory with the vector $\boldsymbol{R}$ of the nuclear axis $R$ given by $\boldsymbol{R}=\boldsymbol{b}+\boldsymbol{v} t$ where $\boldsymbol{b}$ is the impact parameter and $t$ is time (as before, $\boldsymbol{v}$ is the incident velocity). This classical $\boldsymbol{R}$ can be directly related to the corresponding expression $\boldsymbol{R}=\boldsymbol{\rho}+\boldsymbol{Z}$ from (2.6) if we set $\boldsymbol{Z}=\boldsymbol{v} t$ and interpret $\rho$ as the impact parameter. With this interpretation, for example the quantities $\rho^{2 i \nu_{\mathrm{PT}}} \mathcal{A}_{i f}^{(\mathrm{CDW}-4 \mathrm{~B}) \mp}(\rho)$ from the eikonal quantum-mechanical treatment are the transition amplitudes in the four-body impact parameter formulation of the CDW-4B method for double charge exchange (2.1). Here "the four-body impact parameter formulation of the CDW-4B method" should not be confused with the usual impact parameter method (IPM) implemented for double charge exchange in the CDW approximation (as denoted by CDW-IPM) [17,19]. This is the case because the CDW-IPM approximation for double charge exchange is a three-body formalism which uses the product of the two three-body impact-parameter dependent transition amplitudes for each of the two transferred electrons. As such, the CDW-IPM approximation ignores the four-body nature of two electron capture process (2.1) and, in particular, neglects the inter-electron correlation effects.

Overall, the relation (2.123) displays the greatest practical usefulness of the eikonal setting because the product of the two confluent hypergeometric functions in (2.122) for the relative motion of heavy nuclei is first reduced to a single phase $(\mu v \rho)^{2 i \nu_{\mathrm{PT}}}$, which is subsequently shown to disappear altogether from the total cross sections. It is such a complete elimination of the $\boldsymbol{R}$-dependent Coulomb wave functions and their logarithmic phase factors that enormously simplifies the computations of total cross sections for double charge exchange in the CDW-4B method [30,31].

\subsubsection{Symmetric first-order theories: four-body boundary-corrected first Born method, CB1-4B}

Here, for the entrance and exit channels, we choose the distorting potential $w_{i, f}$ in (2.63) as the following short-range interactions:

$$
\begin{gathered}
w_{i}=V_{i}^{\infty}-V_{i, \text { eik }}^{\infty}=Z_{\mathrm{P}}\left(Z_{\mathrm{T}}-2\right)\left(\frac{1}{R}-\frac{1}{r_{i}}\right), \\
w_{f}=V_{f}^{\infty}-V_{f, \text { eik }}^{\infty}=Z_{\mathrm{T}}\left(Z_{\mathrm{P}}-2\right)\left(\frac{1}{R}-\frac{1}{r_{f}}\right) .
\end{gathered}
$$

In other words, the distorting potentials $W_{i, f}$ from (2.63) are of the forms:

$$
W_{i}=V_{i, \text { eik }}^{\infty}=\frac{Z_{\mathrm{P}}\left(Z_{\mathrm{T}}-2\right)}{R}, \quad W_{f}=V_{f, \text { eik }}^{\infty}=\frac{Z_{\mathrm{T}}\left(Z_{\mathrm{P}}-2\right)}{R} .
$$

As a consequence, the perturbations that cause the transitions in the prior and post cross sections are $U_{i}=V_{i}-V_{i, \text { eik }}^{\infty}=V_{\mathrm{PT}}+V_{\mathrm{P} 1}+V_{\mathrm{P} 2}-V_{i, \text { eik }}^{\infty}$ and $U_{f}=V_{f}-V_{f, \text { eik }}^{\infty}=$ $V_{\mathrm{PT}}+V_{\mathrm{T} 1}+V_{\mathrm{T} 2}-V_{f, \text { eik }}^{\infty}$, respectively, or explicitly:

$$
U_{i}=Z_{\mathrm{P}}\left(\frac{2}{R}-\frac{1}{s_{1}}-\frac{1}{s_{2}}\right),
$$




$$
U_{f}=Z_{\mathrm{T}}\left(\frac{2}{R}-\frac{1}{x_{1}}-\frac{1}{x_{2}}\right) .
$$

Inserting $W_{i, f}$ from(2.144) into Eqs. (2.61) and (2.62) for $\chi_{i, f}^{ \pm}$, we have:

$$
\begin{gathered}
\left\{H_{i}+\frac{Z_{\mathrm{P}}\left(Z_{\mathrm{T}}-2\right)}{R}-E_{i}\right\} \chi_{i}^{+}=0, \\
\left\{H_{f}+\frac{Z_{\mathrm{T}}\left(Z_{\mathrm{P}}-2\right)}{R}-E_{f}\right\} \chi_{f}^{-}=0 .
\end{gathered}
$$

Similarly to Eqs. (2.89) and (2.100) from the CDW-4B method, we can use the eikonal relations $R \approx r_{f}$ and $R \approx r_{i}$ in (2.147) and (2.148) via $Z_{\mathrm{P}}\left(Z_{\mathrm{T}}-2\right) / R \approx$ $Z_{\mathrm{P}}\left(Z_{\mathrm{T}}-2\right) / r_{f}$ and $Z_{\mathrm{T}}\left(Z_{\mathrm{P}}-2\right) / R \approx Z_{\mathrm{T}}\left(Z_{\mathrm{P}}-2\right) / r_{i}$, respectively. This separates the variables in Eqs. (2.147) and (2.148) whose solutions $\chi_{i, f}^{ \pm}$with the correct asymptotes $\Phi_{i, f}^{ \pm}$are:

$$
\begin{aligned}
& \chi_{i}^{+}=\Phi_{i} \mathcal{N}^{+}\left(v_{i}\right)_{1} F_{1}\left(-i v_{i}, 1, i k_{i} r_{f}+i \boldsymbol{k}_{i} \cdot \boldsymbol{r}_{f}\right), \chi_{i}^{+} \underset{r_{i} \rightarrow \infty}{\longrightarrow} \Phi_{i}^{+}, \\
& \chi_{f}^{-}=\Phi_{f} \mathcal{N}^{+}\left(v_{f}\right)_{1} F_{1}\left(i v_{f}, 1,-i k_{f} r_{i}-i \boldsymbol{k}_{f} \cdot \boldsymbol{r}_{i}\right), \chi_{f}^{-} \underset{r_{f} \rightarrow \infty}{\longrightarrow} \Phi_{f}^{-},
\end{aligned}
$$

with

$$
\mathcal{N}^{+}\left(v_{i}\right)=\mathrm{e}^{-\pi v_{i} / 2} \Gamma\left(1+i v_{i}\right), \quad \mathcal{N}^{-}\left(v_{f}\right)=\mathrm{e}^{-\pi v_{f} / 2} \Gamma\left(1-i v_{f}\right)
$$

where $v_{i, f}$ are given in (2.27). Then the resulting prior and post forms of the transition amplitudes in the CB1-4B method $[33,34,39,40]$ are summarized as:

$$
\left\{\begin{array}{c}
\text { Prior CB1 - 4B : Transition amplitude } T_{i f}^{(\mathrm{CB} 1-4 \mathrm{~B})-}=\left\langle\chi_{f}^{-}\left|U_{i}\right| \chi_{i}^{+}\right\rangle \\
\bullet U_{i} \text { from }(2.145) \text { and } \chi_{i}^{+} \text {from }(2.149) \\
\bullet \chi_{f}^{-} \text {from }(2.150)
\end{array}\right\},
$$

$$
\left\{\begin{array}{c}
\text { Post CB1 }-4 \mathrm{~B}: \text { Transition amplitude } T_{i f}^{(\mathrm{CB} 1-4 \mathrm{~B})+}=\left\langle\chi_{f}^{-}\left|U_{f}^{\dagger}\right| \chi_{i}^{+}\right\rangle \\
\bullet \chi_{i}^{+} \text {from }(2.149) \\
\bullet U_{f} \text { from (2.146) and } \chi_{f}^{-} \text {from (2.150) }
\end{array}\right\} .
$$

The matrix elements for the prior and post transition amplitudes $T_{i f}^{(\mathrm{CB} 1-4 \mathrm{~B}) \mp}$ take the forms:

$$
\begin{aligned}
T_{i f}^{(\mathrm{CB} 1-4 \mathrm{~B})-} & =\iiint \mathrm{d} \boldsymbol{x}_{1} \mathrm{~d} \boldsymbol{x}_{2} \mathrm{~d} \boldsymbol{r}_{i} \mathrm{e}^{i \boldsymbol{k}_{i} \cdot \boldsymbol{r}_{i}+i \boldsymbol{k}_{f} \cdot \boldsymbol{r}_{f}} \mathcal{L}_{i f}^{(\mathrm{CB} 1-4 \mathrm{~B})-} \mathcal{B}_{i f}^{(\mathrm{CB} 1-4 \mathrm{~B})-}, \\
T_{i f}^{(\mathrm{CB} 1-4 \mathrm{~B})+} & =\iiint \mathrm{d} \boldsymbol{s}_{1} \mathrm{~d} \boldsymbol{s}_{2} \mathrm{~d} \boldsymbol{r}_{f} \mathrm{e}^{i \boldsymbol{k}_{i} \cdot \boldsymbol{r}_{i}+i \boldsymbol{k}_{f} \cdot \boldsymbol{r}_{f}} \mathcal{L}_{i f}^{(\mathrm{CB} 1-4 \mathrm{~B})+} \mathcal{B}_{i f}^{(\mathrm{CB} 1-4 \mathrm{~B})+},
\end{aligned}
$$


where

$$
\begin{aligned}
\mathcal{B}_{i f}^{(\mathrm{CB} 1-4 \mathrm{~B})-}= & \varphi_{f}^{*}\left(\boldsymbol{s}_{1}, \boldsymbol{s}_{2}\right)\left[Z_{\mathrm{P}}\left(\frac{2}{R}-\frac{1}{s_{1}}-\frac{1}{s_{2}}\right)\right] \varphi_{i}\left(\boldsymbol{x}_{1}, \boldsymbol{x}_{2}\right) \\
\mathcal{B}_{i f}^{(\mathrm{CB} 1-4 \mathrm{~B})+}= & \varphi_{f}^{*}\left(\boldsymbol{s}_{1}, \boldsymbol{s}_{2}\right)\left[Z_{\mathrm{T}}\left(\frac{2}{R}-\frac{1}{x_{1}}-\frac{1}{x_{2}}\right)\right] \varphi_{i}\left(\boldsymbol{x}_{1}, \boldsymbol{x}_{2}\right) \\
\mathcal{L}_{i f}^{(\mathrm{CB} 1-4 \mathrm{~B})-}= & \mathcal{L}_{i f}^{(\mathrm{CB} 1-4 \mathrm{~B})+}=\mathcal{N}^{+}\left(v_{i}\right) \mathcal{N}^{-*}\left(v_{f}\right)_{1} F_{1}\left(-i v_{i}, 1, i k_{i} r_{f}+i \boldsymbol{k}_{i} \cdot \boldsymbol{r}_{f}\right) \\
& { }_{1} F_{1}\left(-i v_{f}, 1, i k_{f} r_{i}+i \boldsymbol{k}_{f} \cdot \boldsymbol{r}_{i}\right) .
\end{aligned}
$$

Within the eikonal formalism, the heavy mass limits $M_{\mathrm{P}, \mathrm{T}} \gg 1$ imply:

$$
\begin{aligned}
& \mathcal{L}_{i f}^{(\mathrm{CB} 1-4 \mathrm{~B})-}{ }_{M_{\mathrm{P}, \mathrm{T}} \underset{ }{ } \underset{1}{ }} \underset{ }{ }\left(k_{i} r_{f}+\boldsymbol{k}_{i} \cdot \boldsymbol{r}_{f}\right)^{i v_{i}}\left(k_{f} r_{i}+\boldsymbol{k}_{f} \cdot \boldsymbol{r}_{i}\right)^{i v_{f}} \\
& M_{\mathrm{P}, \mathrm{T}} \underset{\mathbb{\gg}_{1}}{\widetilde{2}}\{\mu(v R-\boldsymbol{v} \cdot \boldsymbol{R})\}^{i v_{i}}\{\mu(v R+\boldsymbol{v} \cdot \boldsymbol{R})\}^{i v_{f}}=(\mu v \rho)^{2 i v_{f}}(v R-\boldsymbol{v} \cdot \boldsymbol{R})^{i \xi}, \\
& \therefore \quad \mathcal{L}_{i f}^{(\mathrm{CB} 1-4 \mathrm{~B})-} \underset{M_{\mathrm{P}, \mathrm{T}} \gg 1}{\approx}(\mu v \rho)^{2 i v_{f}}(v R-\boldsymbol{v} \cdot \boldsymbol{R})^{i \xi}, \\
& \mathcal{L}_{i f}^{(\mathrm{CB} 1-4 \mathrm{~B})+} \underset{M_{\mathrm{P}, \mathrm{T}} \gg 1}{\underset{\sim}{\approx}}\left(k_{i} r_{f}+\boldsymbol{k}_{i} \cdot \boldsymbol{r}_{f}\right)^{i v_{i}}\left(k_{f} r_{i}+\boldsymbol{k}_{f} \cdot \boldsymbol{r}_{i}\right)^{i v_{f}} \\
& M_{\mathrm{P}, \mathrm{T} \gg 1} \underset{{ }^{2}}{\approx}\{\mu(v R-\boldsymbol{v} \cdot \boldsymbol{R})\}^{i v_{i}}\{\mu(v R+\boldsymbol{v} \cdot \boldsymbol{R})\}^{i v_{f}}=(\mu v \rho)^{2 i v_{i}}(v R+\boldsymbol{v} \cdot \boldsymbol{R})^{-i \xi} \text {, } \\
& \therefore \quad \mathcal{L}_{i f}^{(\mathrm{CB} 1-4 \mathrm{~B})+} \underset{M_{\mathrm{P}, \mathrm{T}} \underset{ }{\approx}}{\approx}(\mu v \rho)^{2 i v_{i}}(v R+\boldsymbol{v} \cdot \boldsymbol{R})^{-i \xi}, \\
& \xi=2 \frac{Z_{\mathrm{T}}-Z_{\mathrm{P}}}{v}
\end{aligned}
$$

Using (2.124), the eikonal alternatives to the prior and post transition amplitudes $T_{i f}^{(\mathrm{CB} 1-4 \mathrm{~B})-}$ and $T_{i f}^{(\mathrm{CB} 1-4 \mathrm{~B})+}$ from (2.154) and (2.155) read as:

$$
\begin{aligned}
& T_{i f}^{(\mathrm{CB} 1-4 \mathrm{~B})-}{ }_{(\boldsymbol{\eta})}=\iiint \mathrm{d} \boldsymbol{x}_{1} \mathrm{~d} \boldsymbol{x}_{2} \mathrm{~d} \boldsymbol{R}(\mu v \rho)^{2 i v_{f}}(v \boldsymbol{R}-\boldsymbol{v} \cdot \boldsymbol{R})^{i \xi} \mathcal{D} \mathcal{B}_{i f}^{(\mathrm{CB} 1-4 \mathrm{~B})-}, \\
& T_{i f}^{(\mathrm{CB} 1-4 \mathrm{~B})+}{ }_{(\boldsymbol{\eta})}=\iiint \mathrm{d} \boldsymbol{s}_{1} \mathrm{~d} \boldsymbol{s}_{2} \mathrm{~d} \boldsymbol{R}(\mu v \rho)^{2 i v_{i}}(v R+\boldsymbol{v} \cdot \boldsymbol{R})^{-i \xi} \mathcal{D} \mathcal{B}_{i f}^{(\mathrm{CB} 1-4 \mathrm{~B})+} .
\end{aligned}
$$

Due to (2.159) and (2.160), the entire dependence of the transition amplitudes $T_{i f}^{(\mathrm{CB} 1-4 \mathrm{~B})-}(\boldsymbol{\eta})$ and $T_{i f}^{(\mathrm{CB} 1-4 \mathrm{~B})+}(\boldsymbol{\eta})$ from $(2.162)$ and $(2.163)$ on the inter-nuclear potential $V_{\mathrm{PT}}$ is contained in the Coulomb phases $(\mu v \rho)^{2 i v_{f}}$ and $(\mu v \rho)^{2 i v_{i}}$, respectively. Therefore, the same proof from the CDW-4B method can also be made in the CB1-4B method showing that the eikonal total cross sections $Q_{i f}^{(\mathrm{CB} 1-4 \mathrm{~B})-}$ and $Q_{i f}^{(\mathrm{CB} 1-4 \mathrm{~B})+}$ are unaffected by the phases $(\mu v \rho)^{2 i v_{f}}$ and $(\mu v \rho)^{2 i v_{i}}$. This makes 
$Q_{i f}^{(\mathrm{CB} 1-4 \mathrm{~B}) \mp}$ independent of the inter-nuclear potential $V_{\mathrm{PT}}$ and, therefore, computable from the expressions:

$$
\begin{aligned}
& Q_{i f}^{(\mathrm{CB} 1-4 \mathrm{~B}) \mp}\left(a_{0}^{2}\right)=\int \mathrm{d} \eta\left|\frac{T_{i f}^{(\mathrm{CB} 1-4 \mathrm{~B}) \mp}(\boldsymbol{\eta})}{2 \pi v}\right|^{2}, \\
& =\int \mathrm{d} \eta\left|\frac{R_{i f}^{(\mathrm{CB} 1-4 \mathrm{~B}) \mp}(\eta)}{2 \pi v}\right|^{2},
\end{aligned}
$$

where

$$
\begin{aligned}
& R_{i f}^{(\mathrm{CB} 1-4 \mathrm{~B})-}(\boldsymbol{\eta})=\iiint \mathrm{d} \boldsymbol{x}_{1} \mathrm{~d} \boldsymbol{x}_{2} \mathrm{~d} \boldsymbol{R}(v R-\boldsymbol{v} \cdot \boldsymbol{R})^{i \xi} \mathcal{D} \mathcal{B}_{i f}^{(\mathrm{CB} 1-4 \mathrm{~B})-}, \\
& R_{i f}^{(\mathrm{CB} 1-4 \mathrm{~B})+}(\boldsymbol{\eta})=\iiint \mathrm{d} \boldsymbol{s}_{1} \mathrm{~d} \boldsymbol{s}_{2} \mathrm{~d} \boldsymbol{R}(v R+\boldsymbol{v} \cdot \boldsymbol{R})^{-i \xi} \mathcal{D} \mathcal{B}_{i f}^{(\mathrm{CB} 1-4 \mathrm{~B})+} .
\end{aligned}
$$

Note that the exact functions $\mathcal{L}_{i f}^{(\mathrm{CB} 1-4 \mathrm{~B})-}$ and $\mathcal{L}_{i f}^{(\mathrm{CB} 1-4 \mathrm{~B})+}$ are equal to each other, as per (2.158), and so are their eikonal forms (2.159) and (2.160) due to the relations:

$$
(\mu v \rho)^{2 i v_{f}}(v R-\boldsymbol{v} \cdot \boldsymbol{R})^{i \xi}=(\mu v \rho)^{2 i v_{i}}(v R+\boldsymbol{v} \cdot \boldsymbol{R})^{-i \xi} .
$$

In other words, either $(\mu v \rho)^{2 i v_{f}}(v R-\boldsymbol{v} \cdot \boldsymbol{R})^{i \xi}$ or $(\mu v \rho)^{2 i v_{i}}(v \boldsymbol{R}+\boldsymbol{v} \cdot \boldsymbol{R})^{-i \xi}$ can be employed in $\mathcal{L}_{i f}^{(\mathrm{CB} 1-4 \mathrm{~B})-}$ from $T_{i f}^{(\mathrm{CB} 1-4 \mathrm{~B})-}$ or in $\mathcal{L}_{i f}^{(\mathrm{CB} 1-4 \mathrm{~B})+}$ from $T_{i f}^{(\mathrm{CB} 1-4 \mathrm{~B})+}$ instead of the single product of the two full Coulomb waves (2.158). The only reason for stating the two equivalent eikonal expressions (2.159) and (2.160) is to exhibit the ease in computations of differential cross sections when either $Z_{\mathrm{P}}$ or $Z_{\mathrm{T}}$ is equal to 2 . Thus, for $Z_{\mathrm{T}}=2$ and $Z_{\mathrm{P}}$ arbitrary, we have $v_{i}=0$ and (2.160) should be used. Conversely, for $Z_{\mathrm{T}}$ arbitrary and $Z_{\mathrm{P}}=2$, it follows that $v_{f}=0$ and (2.159) is preferred. The rationale for these preferences is clear from (2.168), where for either $Z_{\mathrm{P}}=2$ or $Z_{\mathrm{T}}=2$, only one Coulomb logarithmic phase remains for the relative motion of heavy particles, namely $(v R-\boldsymbol{v} \cdot \boldsymbol{R})^{i \xi}$ [for $v_{f}=0$ ] or $(v R+\boldsymbol{v} \cdot \boldsymbol{R})^{-i \xi}\left[\right.$ for $v_{i}=0$ ]. Thus, for either $Z_{\mathrm{P}}=2$ or $Z_{\mathrm{T}}=2$, the differential cross sections in the CB1-4B method are directly proportional to the squared moduli of $\left|R_{i f}^{(\mathrm{CB} 1-4 \mathrm{~B}) \mp}(\eta)\right|^{2}$. In these two particular cases, the same matrix elements $R_{i f}^{(\mathrm{CB} 1-4 \mathrm{~B}) \mp}(\eta)$ for total cross sections can also be used for differential cross sections. These special circumstances avoid computations of differential cross sections in the CB1-4B method by the standard, highly oscillatory numerical integrations (in the interval $0 \leq \rho \leq+\infty$ ) over the integrand comprised of the product of the factors $\rho^{1+2 i v_{f, i}}$ with a Bessel function of the first kind and the $\rho$-dependent transition amplitudes $\mathcal{A}_{i f}^{(\mathrm{CB} 1-4 \mathrm{~B}) \mp}(\rho)$.

In Refs. [36-38], the four-body boundary-corrected first Born method, or CB1$4 \mathrm{~B}$, has alternatively been called the four-body Coulomb-Born distorted (CBDW-4B) method because therein the computations make use of the full Coulomb wave functions for the relative motion of heavy nuclei. However, the total cross sections from the CB14B method, employing the logarithmic Coulomb phase factors for the relative motion 
of heavy nuclei, fully agree with those from the CBDW-4B method, as has explicitly been shown in computations on single capture (see section 4 ), and this should also be true for double capture.

\subsubsection{Asymmetric second-order theories: four-body boundary-corrected continuum intermediate states method, BCIS-4B}

The BCIS-4B method [31] is a merger of the CDW-4B and CB1-4B methods. The prior BCIS-4B method uses the CB1-4B and CDW-4B methods in the entrance and exit channels, respectively. Conversely, the post BCIS-4B method employs the CB1$4 \mathrm{~B}$ and CDW-4B methods in the exit and entrance channels, respectively. Thus, the joint charter for the prior and post forms of the BCIS-4B method runs as follows:

$$
\left\{\begin{array}{c}
\text { Prior BCIS - 4B : Transition amplitude } T_{i f}^{(\mathrm{BCIS}-4 \mathrm{~B})-}=\left\langle\chi_{f}^{-}\left|U_{i}\right| \chi_{i}^{+}\right\rangle \\
\bullet U_{i} \text { from }(2.145) \text { and } \chi_{i}^{+} \text {from (2.149) } \\
\bullet \chi_{f}^{-} \text {from }(2.102)
\end{array}\right\},
$$

$$
\left\{\begin{array}{c}
\text { Post BCIS - 4B : Transition amplitude } T_{i f}^{(\mathrm{BCIS}-4 \mathrm{~B})+}=\left\langle\chi_{f}^{-}\left|U_{f}^{\dagger}\right| \chi_{i}^{+}\right\rangle \\
\bullet \chi_{i}^{+} \text {from }(2.93) \\
\bullet U_{f} \text { from Eq. (2.146) and } \chi_{f}^{-} \text {from Eq. (2.150) }
\end{array}\right\} .
$$

Hence, this prescription has the following explicit $T$-matrix elements:

$$
\begin{aligned}
T_{i f}^{(\mathrm{BCIS}-4 \mathrm{~B})-} & =N_{\mathrm{T}}^{2} C_{\mathrm{T}} \iiint \mathrm{d} \boldsymbol{x}_{1} \mathrm{~d} \boldsymbol{x}_{2} \mathrm{~d} \boldsymbol{r}_{i} \mathrm{e}^{i \boldsymbol{k}_{i} \cdot \boldsymbol{r}_{i}+i \boldsymbol{k}_{f} \cdot \boldsymbol{r}_{f}} \mathcal{L}_{i f}^{(\mathrm{BCIS}-4 \mathrm{~B})-} \mathcal{B}_{i f}^{(\mathrm{BCIS}-4 \mathrm{~B})-}, \\
T_{i f}^{(\mathrm{BCIS}-4 \mathrm{~B})+} & =N_{\mathrm{P}}^{2} C_{\mathrm{P}} \iiint \mathrm{d} \boldsymbol{s}_{1} \mathrm{~d} \boldsymbol{s}_{2} \mathrm{~d} \boldsymbol{r}_{f} \mathrm{e}^{i \boldsymbol{k}_{i} \cdot \boldsymbol{r}_{i}+i \boldsymbol{k}_{f} \cdot \boldsymbol{r}_{f}} \mathcal{L}_{i f}^{(\mathrm{BCIS}-4 \mathrm{~B})+} \mathcal{B}_{i f}^{(\mathrm{BCIS}-4 \mathrm{~B})+},
\end{aligned}
$$

where

$$
\begin{aligned}
C_{\mathrm{P}}=\mu_{i}^{-2 i \nu_{\mathrm{P}}}, & C_{\mathrm{T}}=\mu_{f}^{-2 i v_{\mathrm{T}}}, N_{\mathrm{P}}=N^{+}\left(\nu_{\mathrm{P}}\right), N_{\mathrm{T}}=N^{-*}\left(v_{\mathrm{T}}\right), \\
\mathcal{B}_{i f}^{(\mathrm{BCIS}-4 \mathrm{~B})-}= & \varphi_{f}^{*}\left(\boldsymbol{s}_{1}, \boldsymbol{s}_{2}\right)\left[Z_{\mathrm{P}}\left(\frac{2}{R}-\frac{1}{s_{1}}-\frac{1}{s_{2}}\right)\right] \varphi_{i}\left(\boldsymbol{x}_{1}, \boldsymbol{x}_{2}\right) \\
& \times_{1} F_{1}\left(i v_{\mathrm{T}}, 1, i v x_{1}+i \boldsymbol{v} \cdot \boldsymbol{x}_{1}\right){ }_{1} F_{1}\left(i v_{\mathrm{T}}, 1, i v x_{2}+i \boldsymbol{v} \cdot \boldsymbol{x}_{2}\right), \\
\mathcal{B}_{i f}^{(\mathrm{BCIS}-4 \mathrm{~B})+}= & \varphi_{f}^{*}\left(\boldsymbol{s}_{1}, \boldsymbol{s}_{2}\right)\left[Z_{\mathrm{T}}\left(\frac{2}{R}-\frac{1}{x_{1}}-\frac{1}{x_{2}}\right)\right] \varphi_{i}\left(\boldsymbol{x}_{1}, \boldsymbol{x}_{2}\right)
\end{aligned}
$$




$$
\begin{aligned}
\mathcal{L}_{i f}^{(\mathrm{BCIS}-4 \mathrm{~B})-}= & \mathcal{N}^{+}\left(v_{i}\right) \mathcal{N}^{-*}\left(i v_{\mathrm{P}}, 1, i v s_{1}+i \boldsymbol{v} \cdot \boldsymbol{s}_{1}\right){ }_{1} F_{1}\left(i v_{\mathrm{P}}, 1, i v s_{2}+i \boldsymbol{v} \cdot \boldsymbol{s}_{2}\right), \\
& \times{ }_{1} F_{1}\left(-i v_{i}, 1, i k_{i} r_{f}+i \boldsymbol{k}_{i} \cdot \boldsymbol{r}_{f}\right){ }_{1} F_{1}\left(-i v_{\mathrm{PT}}, 1, i k_{f} r_{i}+i \boldsymbol{k}_{f} \cdot \boldsymbol{r}_{i}\right), \\
\mathcal{L}_{i f}^{(\mathrm{BCIS}-4 \mathrm{~B})+}= & \mathcal{N}^{+}\left(\nu_{\mathrm{PT}}\right) \mathcal{N}^{-*}\left(v_{f}\right) \\
& \times{ }_{1} F_{1}\left(-i v_{\mathrm{PT}}, 1, i k_{i} r_{f}+i \boldsymbol{k}_{i} \cdot \boldsymbol{r}_{f}\right){ }_{1} F_{1}\left(-i v_{f}, 1, i k_{f} r_{i}+i \boldsymbol{k}_{f} \cdot \boldsymbol{r}_{i}\right) .
\end{aligned}
$$

Using the eikonal hypothesis, with its heavy mass limits $M_{\mathrm{P}, \mathrm{T}} \gg 1$, we have:

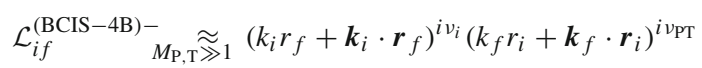

$$
\begin{aligned}
& M_{\mathrm{P}, \mathrm{T}} \underset{{ }_{1} 1}{\approx}\{\mu(v R-\boldsymbol{v} \cdot \boldsymbol{R})\}^{i v_{i}}\{\mu(v R+\boldsymbol{v} \cdot \boldsymbol{R})\}^{i \nu_{\mathrm{PT}}}=(\mu v \rho)^{2 i \nu_{\mathrm{PT}}}(v R-\boldsymbol{v} \cdot \boldsymbol{R})^{-2 i \nu_{\mathrm{P}}},
\end{aligned}
$$

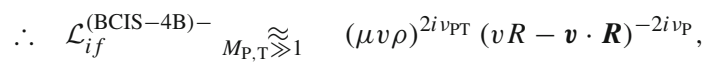

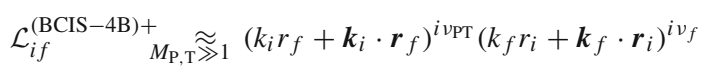

$$
\begin{aligned}
& M_{\mathrm{P}, \mathrm{T}} \underset{{ }^{\prime} 1}{\widetilde{2}}\{\mu(v R-\boldsymbol{v} \cdot \boldsymbol{R})\}^{i \nu_{\mathrm{PT}}}\{\mu(v R+\boldsymbol{v} \cdot \boldsymbol{R})\}^{i \nu_{f}}=(\mu v \rho)^{2 i \nu_{\mathrm{PT}}}(v R+\boldsymbol{v} \cdot \boldsymbol{R})^{-2 i \nu_{\mathrm{T}}}, \\
& \therefore \quad \mathcal{L}_{i f}^{(\mathrm{BCIS}-4 \mathrm{~B})+} M_{\mathrm{P}, \mathrm{T}} \underset{\gg 1}{\approx}(\mu v \rho)^{2 i \nu_{\mathrm{PT}}}(v R+\boldsymbol{v} \cdot \boldsymbol{R})^{-2 i v_{\mathrm{T}}} .
\end{aligned}
$$

Employing (2.124), the eikonal versions of the transition amplitudes $T_{i f}^{\text {(BCIS-4B)- }}$ and $T_{i f}^{(\mathrm{BCIS}-4 \mathrm{~B})+}$ from (2.171) and (2.172) become:

$$
\begin{aligned}
T_{i f}^{(\mathrm{BCIS}-4 \mathrm{~B})-}{ }_{(\boldsymbol{\eta})=} & N_{\mathrm{T}}^{2} C_{\mathrm{T}}^{\prime} \iiint \mathrm{d} \boldsymbol{x}_{1} \mathrm{~d} \boldsymbol{x}_{2} \mathrm{~d} \boldsymbol{R} \rho^{2 i \nu_{\mathrm{PT}}} \\
& \times(v R-\boldsymbol{v} \cdot \boldsymbol{R})^{-2 i v_{\mathrm{P}}} \mathcal{D} \mathcal{B}_{i f}^{(\mathrm{BCIS}-4 \mathrm{~B})-}, \\
T_{i f}^{(\mathrm{BCIS}-4 \mathrm{~B})+}(\boldsymbol{\eta})= & N_{\mathrm{P}}^{2} C_{\mathrm{P}}^{\prime} \iiint \mathrm{d} \boldsymbol{s}_{1} \mathrm{~d} \boldsymbol{s}_{2} \mathrm{~d} \boldsymbol{R} \rho^{2 i \nu_{\mathrm{PT}}} \\
& \times(v R+\boldsymbol{v} \cdot \boldsymbol{R})^{-2 i v_{\mathrm{T}}} \mathcal{D} \mathcal{B}_{i f}^{(\mathrm{BCIS}-4 \mathrm{~B})+},
\end{aligned}
$$

where

$$
\left.\begin{array}{c}
C_{\mathrm{P}}^{\prime}=C_{\mathrm{P}}(\mu v)^{2 i \nu_{\mathrm{PT}}}, \quad C_{\mathrm{P}}^{\prime} \underset{M_{\mathrm{P}, \mathrm{T} \gg 1}}{\approx} v^{2 i \nu_{\mathrm{PT}}} \mu^{2 i\left(\nu_{\mathrm{PT}}-\nu_{\mathrm{P}}\right)} \\
C_{\mathrm{T}}^{\prime}=C_{\mathrm{T}}(\mu v)^{2 i \nu_{\mathrm{PT}}}, \quad C_{\mathrm{T}}^{\prime} \underset{\substack{M_{\mathrm{P}, \mathrm{T}} \gg 1 \\
\approx}}{\approx 2 i \nu_{\mathrm{PT}}} \mu^{2 i\left(\nu_{\mathrm{PT}}-\nu_{\mathrm{T}}\right)}
\end{array}\right\} .
$$


Because of the relations (2.178) and (2.179), the eikonal transition amplitudes $T_{i f}^{(\mathrm{BCIS}-4 \mathrm{~B})-}(\boldsymbol{\eta})$ and $T_{i f}^{(\mathrm{BCIS}-4 \mathrm{~B})+}(\boldsymbol{\eta})$ in the BCIS-4B method depend on the internuclear potential $V_{\mathrm{PT}}$ only through the single Coulomb phases, $(\mu v \rho)^{2 i \nu_{\mathrm{PT}}}$, as is the case with the CDW-4B method. This latter phase disappears from the corresponding eikonal total cross sections $Q_{i f}^{(\mathrm{BCIS}-4 \mathrm{~B}) \mp}$ that are, therefore, independent of the the inter-nuclear potential $V_{\mathrm{PT}}$ and, as such, computable from the expressions:

$$
\begin{aligned}
Q_{i f}^{(\mathrm{BCIS}-4 \mathrm{~B}) \mp}\left(a_{0}^{2}\right) & =\int \mathrm{d} \boldsymbol{\eta}\left|\frac{T_{i f}^{(\mathrm{BCIS}-4 \mathrm{~B}) \mp}(\boldsymbol{\eta})}{2 \pi v}\right|^{2}, \\
& =\int \mathrm{d} \boldsymbol{\eta}\left|\frac{R_{i f}^{(\mathrm{BCIS}-4 \mathrm{~B}) \mp}(\boldsymbol{\eta})}{2 \pi v}\right|^{2},
\end{aligned}
$$

where

$$
\begin{aligned}
R_{i f}^{(\mathrm{BCIS}-4 \mathrm{~B})-}(\boldsymbol{\eta})= & N_{\mathrm{T}}^{2} C_{\mathrm{T}}^{\prime} \iiint \mathrm{d} \boldsymbol{x}_{1} \mathrm{~d} \boldsymbol{x}_{2} \mathrm{~d} \boldsymbol{R} \\
& \times(v R-\boldsymbol{v} \cdot \boldsymbol{R})^{-2 i \nu_{\mathrm{P}}} \mathcal{D} \mathcal{B}_{i f}^{(\mathrm{BCIS}-4 \mathrm{~B})-}, \\
R_{i f}^{(\mathrm{BCIS}-4 \mathrm{~B})+}(\boldsymbol{\eta})= & N_{\mathrm{P}}^{2} C_{\mathrm{P}}^{\prime} \iiint \mathrm{d} \boldsymbol{s}_{1} \mathrm{~d} \boldsymbol{s}_{2} \mathrm{~d} \boldsymbol{R} \\
& \times(v R+\boldsymbol{v} \cdot \boldsymbol{R})^{-2 i v_{\mathrm{T}}} \mathcal{D} \mathcal{B}_{i f}^{(\mathrm{BCIS}-4 \mathrm{~B})+} .
\end{aligned}
$$

Note that in Refs. [49-53] for double electron capture processes, the four-body boundary-corrected continuum intermediate state method based upon the full Coulomb wave function for the relative motion of heavy nuclei has been denoted by the acronym BCCIS-4B. Unexpectedly, however, the total cross sections from the BCCIS-4B method [49-53] do not coincide with those obtained employing by the BCIS-4B method in terms of the corresponding logarithmic Coulomb phase factors for the relative motion of heavy nuclei $[1,3,32]$. We analyzed this discrepancy by performing a new detailed computation whose results will be reported very soon (see also the pertinenet comment on p. 1437 in Ref [3]).

\subsubsection{Asymmetric second-order theories: four-body Born distorted wave method, BDW-4B}

The BDW-4B method [41,42] is also a hybrid of the CDW-4B and CB1-4B methods. In the BDW-4B method, the role of the CDW-4B and CB1-4B methods is inter-exchanged relative to the BCIS-4B method. Namely, the prior BDW-4B method employs the CDW-4B and CB1-4B methods in the entrance and exit channels, respectively. On the other hand, the post BDW-4B method uses the CDW-4B and CB1-4B methods in the exit and entrance, respectively. With this at hand, the prior and post forms of the 
BDW-4B method are specified by:

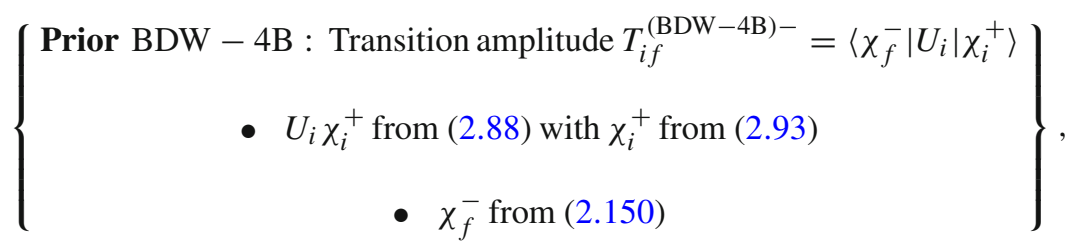

$$
\left\{\begin{array}{c}
\text { Post BDW - 4B : Transition amplitude } T_{i f}^{(\mathrm{BDW}-4 \mathrm{~B})+}=\left\langle\chi_{f}^{-}\left|U_{f}^{\dagger}\right| \chi_{i}^{+}\right\rangle \\
\bullet \chi_{i}^{+} \text {from (2.149) } \\
\bullet U_{f} \chi_{f}^{-} \text {from (2.99) with } \chi_{f}^{-} \text {from (2.102) }
\end{array}\right\} .
$$

The corresponding matrix elements in the transition amplitudes read as:

$$
\begin{aligned}
& T_{i f}^{(\mathrm{BDW}-4 \mathrm{~B})-}=-N_{\mathrm{P}}^{2} C_{\mathrm{P}} \iiint \mathrm{d} \boldsymbol{x}_{1} \mathrm{~d} \boldsymbol{x}_{2} \mathrm{~d} \boldsymbol{r}_{i} \mathrm{e}^{i \boldsymbol{k}_{i} \cdot \boldsymbol{r}_{i}+i \boldsymbol{k}_{f} \cdot \boldsymbol{r}_{f}} \mathcal{L}_{i f}^{(\mathrm{BDW}-4 \mathrm{~B})-} \mathcal{B}_{i f}^{(\mathrm{BDW}-4 \mathrm{~B})-}, \\
& T_{i f}^{\mathrm{BDW}(-4 \mathrm{~B})+}=-N_{\mathrm{T}}^{2} C_{\mathrm{T}} \iiint \mathrm{d} \boldsymbol{s}_{1} \mathrm{~d} \boldsymbol{s}_{2} \mathrm{~d} \boldsymbol{r}_{f} \mathrm{e}^{i \boldsymbol{k}_{i} \cdot \boldsymbol{r}_{i}+i \boldsymbol{k}_{f} \cdot \boldsymbol{r}_{f}} \mathcal{L}_{i f}^{(\mathrm{BDW}-4 \mathrm{~B})+} \mathcal{B}_{i f}^{(\mathrm{BDW}-4 \mathrm{~B})+},
\end{aligned}
$$

where

$$
\begin{aligned}
& \mathcal{B}_{i f}^{(\mathrm{BDW}-4 \mathrm{~B})-}=\varphi_{f}^{*}\left(\boldsymbol{s}_{1}, \boldsymbol{s}_{2}\right) \\
& \quad \times\left\{{ }_{1} F_{1}\left(i v_{\mathrm{P}}, 1, i v s_{2}+i \boldsymbol{v} \cdot \boldsymbol{s}_{2}\right) \nabla_{x_{1}} \varphi_{i}\left(\boldsymbol{x}_{1}, \boldsymbol{x}_{2}\right) \cdot \nabla_{s_{1} 1} F_{1}\left(i v_{\mathrm{P}}, 1, i v s_{1}+i \boldsymbol{v} \cdot \boldsymbol{s}_{1}\right)\right. \\
& \left.\quad+{ }_{1} F_{1}\left(i v_{\mathrm{P}}, 1, i v s_{1}+i \boldsymbol{v} \cdot \boldsymbol{s}_{1}\right) \nabla_{x_{2}} \varphi_{i}\left(\boldsymbol{x}_{1}, \boldsymbol{x}_{2}\right) \cdot \nabla_{s_{2} 1} F_{1}\left(i v_{\mathrm{P}}, 1, i v s_{2}+i \boldsymbol{v} \cdot \boldsymbol{s}_{2}\right)\right\} \\
& \mathcal{B}_{i f}^{(\mathrm{BDW}-4 \mathrm{~B})+}=\varphi_{i}\left(\boldsymbol{x}_{1}, \boldsymbol{x}_{2}\right) \\
& \quad \times\left\{{ }_{1} F_{1}\left(i v_{\mathrm{T}}, 1, i v x_{2}+i \boldsymbol{v} \cdot \boldsymbol{x}_{2}\right) \nabla_{s_{1}} \varphi_{f}^{*}\left(\boldsymbol{s}_{1}, \boldsymbol{s}_{2}\right) \cdot \nabla_{x_{1} 1} F_{1}\left(i v_{\mathrm{T}}, 1, i v x_{1}+i \boldsymbol{v} \cdot \boldsymbol{x}_{1}\right)\right. \\
& \left.\quad+{ }_{1} F_{1}\left(i v_{\mathrm{T}}, 1, i v x_{1}+i \boldsymbol{v} \cdot \boldsymbol{x}_{1}\right) \nabla_{s_{2}} \varphi_{f}^{*}\left(\boldsymbol{s}_{1}, \boldsymbol{s}_{2}\right) \cdot \nabla_{x_{2} 1} F_{1}\left(i v_{\mathrm{T}}, 1, i v x_{2}+i \boldsymbol{v} \cdot \boldsymbol{x}_{2}\right)\right\}, \\
& \mathcal{L}_{i f}^{(\mathrm{BDW}-4 \mathrm{~B})-}=\mathcal{N}^{+}\left(v_{\mathrm{PT}}\right) \mathcal{N}^{-*}\left(v_{f}\right) \\
& \quad \times{ }_{1} F_{1}\left(-i v_{\mathrm{PT}}, 1, i k_{i} \boldsymbol{r}_{f}+i \boldsymbol{k}_{i} \cdot \boldsymbol{r}_{f}\right){ }_{1} F_{1}\left(-i v_{f}, 1, i k_{f} r_{i}+i \boldsymbol{k}_{f} \cdot \boldsymbol{r}_{i}\right), \\
& \mathcal{L}_{i f}^{(\mathrm{BDW}-4 \mathrm{~B})+}=\mathcal{N}^{+}\left(v_{i}\right) \mathcal{N}^{-*}\left(v_{\mathrm{PT}}\right) \\
& \quad \times{ }_{1} F_{1}\left(-i v_{i}, 1, i k_{i} r_{f}+i \boldsymbol{k}_{i} \cdot \boldsymbol{r}_{f}\right){ }_{1} F_{1}\left(-i v_{\mathrm{PT}}, 1, i k_{f} r_{i}+i \boldsymbol{k}_{f} \cdot \boldsymbol{r}_{i}\right) .
\end{aligned}
$$


Here, the $\mathcal{L}$-functions from the prior and post BDW-4B method are identical to those from the post and prior BCIS-4B methods, respectively:

$$
\mathcal{L}_{i f}^{(\mathrm{BDW}-4 \mathrm{~B})-}=\mathcal{L}_{i f}^{(\mathrm{BCIS}-4 \mathrm{~B})+}, \quad \mathcal{L}_{i f}^{(\mathrm{BDW}-4 \mathrm{~B})+}=\mathcal{L}_{i f}^{(\mathrm{BCIS}-4 \mathrm{~B})-},
$$

where $\mathcal{L}_{i f}^{(\mathrm{BCIS}-4 \mathrm{~B})-}$ and $\mathcal{L}_{i f}^{(\mathrm{BCIS}-4 \mathrm{~B})+}$ are given by (2.176) and (2.177), respectively. This implies, by reference to (2.178) and (2.179) for the eikonalized motions of the $\mathrm{P}$ and $\mathrm{T}$ nuclei, that within the heavy mass limits $M_{\mathrm{P}, \mathrm{T}} \gg 1$, we have:

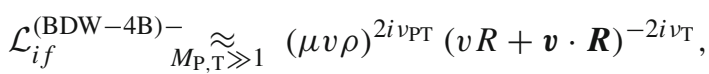

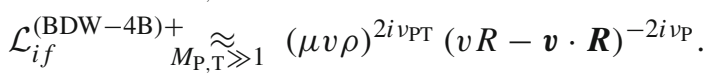

With the help of (2.124), the eikonal total transition amplitudes $T_{i f}^{(\mathrm{BDW}-4 \mathrm{~B}) \mp}(\boldsymbol{\eta})$ are reduced to:

$$
\begin{aligned}
T_{i f}^{(\mathrm{BDW}-4 \mathrm{~B})-}{ }_{(\boldsymbol{\eta})=} & -N_{\mathrm{P}}^{2} C_{\mathrm{P}}^{\prime} \iiint \mathrm{d} \boldsymbol{x}_{1} \mathrm{~d} \boldsymbol{x}_{2} \mathrm{~d} \boldsymbol{R} \rho^{2 i \nu_{\mathrm{PT}}} \\
& \times(v R+\boldsymbol{v} \cdot \boldsymbol{R})^{-2 i \nu_{\mathrm{T}}} \mathcal{D} \mathcal{B}_{i f}^{(\mathrm{BDW}-4 \mathrm{~B})-}, \\
T_{i f}^{(\mathrm{BDW}-4 \mathrm{~B})+}{ }_{(\boldsymbol{\eta})=} & -N_{\mathrm{T}}^{2} C_{\mathrm{T}}^{\prime} \iiint \mathrm{d} \boldsymbol{s}_{1} \mathrm{~d} \boldsymbol{s}_{2} \mathrm{~d} \boldsymbol{R} \rho^{2 i \nu_{\mathrm{PT}}} \\
& \times(v R-\boldsymbol{v} \cdot \boldsymbol{R})^{-2 i \nu_{\mathrm{P}}} \mathcal{D} \mathcal{B}_{i f}^{(\mathrm{BDW}-4 \mathrm{~B})+} .
\end{aligned}
$$

Thus, in the BDW-4B method, the Coulomb phase $(\mu v \rho)^{2 i \nu_{\mathrm{PT}}}$ is the only term due to the inter-nuclear potential $V_{\mathrm{PT}}$ in the eikonal transition amplitudes $T_{i f}^{(\mathrm{BDW}-4 \mathrm{~B})-}(\boldsymbol{\eta})$ and $T_{i f}^{(\mathrm{BDW}-4 \mathrm{~B})+}(\boldsymbol{\eta})$ from (2.198) and (2.199). This remaining phase vanishes from the eikonal total cross sections $Q_{i f}^{(\mathrm{BDW}-4 \mathrm{~B}) \mp}$ that, therefore, become independent of the inter-nuclear potential $V_{\mathrm{PT}}$ :

$$
\begin{aligned}
Q_{i f}^{(\mathrm{BDW}-4 \mathrm{~B}) \mp}\left(a_{0}^{2}\right) & =\int \mathrm{d} \eta\left|\frac{T_{i f}^{(\mathrm{BDW}-4 \mathrm{~B}) \mp}(\eta)}{2 \pi v}\right|^{2}, \\
& =\int \mathrm{d} \eta\left|\frac{R_{i f}^{(\mathrm{BDW}-4 \mathrm{~B}) \mp}(\eta)}{2 \pi v}\right|^{2},
\end{aligned}
$$

where

$$
\begin{aligned}
R_{i f}^{(\mathrm{BDW}-4 \mathrm{~B})-}(\boldsymbol{\eta})=- & N_{\mathrm{P}}^{2} C_{\mathrm{P}}^{\prime} \iiint \mathrm{d} \boldsymbol{x}_{1} \mathrm{~d} \boldsymbol{x}_{2} \mathrm{~d} \boldsymbol{R} \\
& \times(v R+\boldsymbol{v} \cdot \boldsymbol{R})^{-2 i \nu_{\mathrm{T}}} \mathcal{D} \mathcal{B}_{i f}^{(\mathrm{BDW}-4 \mathrm{~B})-}
\end{aligned}
$$




$$
\begin{aligned}
R_{i f}^{(\mathrm{BDW}-4 \mathrm{~B})+}(\boldsymbol{\eta})=- & N_{\mathrm{T}}^{2} C_{\mathrm{T}}^{\prime} \iiint \mathrm{d} \boldsymbol{s}_{1} \mathrm{~d} \boldsymbol{s}_{2} \mathrm{~d} \boldsymbol{R} \\
& \times(v R-\boldsymbol{v} \cdot \boldsymbol{R})^{-2 i \nu_{\mathrm{P}}} \mathcal{D} \mathcal{B}_{i f}^{(\mathrm{BDW}-4 \mathrm{~B})+} .
\end{aligned}
$$

\subsubsection{Asymmetric second-order theories: four-body continuum distorted wave eikonal initial/final state methods, CDW-EIS/EFS-4B}

The CDW-EIS-4B and CDW-EFS-4B methods [47] are the asymmetric approximations to the post and prior versions of the CDW-4B method, respectively. The CDW-EIS-4B method eikonalizes the motions of the two electrons in the initial scattering states $\chi_{i}^{+}$, using its asymptote (2.105), whereas $U_{f} \chi_{f}^{-}$is the same as (2.99) in the post version of the CDW-4B method. Similarly, the CDW-EFS-4B method replaces the two full electronic Coulomb wave functions by their asymptotes (logarithmic phase factors) in the final scattering state $\chi_{f}^{-}$, according to (2.106), while preserving the intact $U_{i} \chi_{i}^{-}$from (2.88) in the prior version of the CDW-4B method. Thus, the schemes for the CDW-EIS-4B and CDW-EFS-4B methods run as follows:

$$
\left\{\begin{array}{c}
\text { CDW - EIS - 4B : Transition amplitude } T_{i f}^{(\mathrm{CDW}-\mathrm{EIS}-4 \mathrm{~B})+}=\left\langle\chi_{f}^{-}\left|U_{f}^{\dagger}\right| \chi_{i}^{+}\right\rangle \\
\bullet \chi_{i}^{+} \text {from (2.105) } \\
\bullet U_{f}\left|\chi_{f}^{-}\right\rangle \text {from (2.99) with } \chi_{f}^{-} \text {from (2.102) }
\end{array}\right\},
$$

$$
\left\{\begin{array}{c}
\mathrm{CDW}-\mathrm{EFS}-4 \mathrm{~B}: \text { Transition amplitude } T_{i f}^{(\mathrm{CDW}-\mathrm{EFS}-4 \mathrm{~B})-}=\left\langle\chi_{f}^{-}\left|U_{i}\right| \chi_{i}^{+}\right\rangle \\
\bullet U_{i} \chi_{i}^{+} \text {from (2.88) with } \chi_{i}^{+} \text {from (2.93) } \\
\bullet \chi_{f}^{-} \text {from Eq. (2.106) }
\end{array}\right\} .
$$

The associated transition amplitudes $T_{i f}^{(\mathrm{CDW}-\mathrm{EIS}-4 \mathrm{~B})+}$ and $T_{i f}^{(\mathrm{CDW}-\mathrm{EFS}-4 \mathrm{~B})-}$ have the forms:

$$
\begin{aligned}
T_{i f}^{(\mathrm{CDW}-\mathrm{EIS}-4 \mathrm{~B})+} & =-N_{\mathrm{T}}^{2} C_{\mathrm{T}} \iiint \mathrm{d} \boldsymbol{s}_{1} \mathrm{~d} \boldsymbol{s}_{2} \mathrm{~d} \boldsymbol{r}_{f} \mathrm{e}^{i \boldsymbol{k}_{i} \cdot \boldsymbol{r}_{i}+i \boldsymbol{k}_{f} \cdot \boldsymbol{r}_{f}} \\
& \times \mathcal{L}_{i f}^{(\mathrm{CDW}-\mathrm{EIS}-4 \mathrm{~B})+} \mathcal{B}_{i f}^{(\mathrm{CDW}-\mathrm{EIS}-4 \mathrm{~B})+}, \\
T_{i f}^{(\mathrm{CDW}-\mathrm{EFS}-4 \mathrm{~B})-} & =-N_{\mathrm{P}}^{2} C_{\mathrm{P}} \iiint \mathrm{d} \boldsymbol{x}_{1} \mathrm{~d} \boldsymbol{x}_{2} \mathrm{~d} \boldsymbol{r}_{i} \mathrm{e}^{i \boldsymbol{k}_{i} \cdot \boldsymbol{r}_{i}+i \boldsymbol{k}_{f} \cdot \boldsymbol{r}_{f}} \\
& \times \mathcal{L}_{i f}^{(\mathrm{CDW}-\mathrm{EFS}-4 \mathrm{~B})-} \mathcal{B}_{i f}^{(\mathrm{CDW}-\mathrm{EFS}-4 \mathrm{~B})-},
\end{aligned}
$$

where 


$$
\begin{aligned}
& \mathcal{B}_{i f}^{(\mathrm{CDW}-\mathrm{EIS}-4 \mathrm{~B})+}=\varphi_{i}\left(\boldsymbol{x}_{1}, \boldsymbol{x}_{2}\right)\left(v s_{1}+\boldsymbol{v} \cdot \boldsymbol{s}_{1}\right)^{-i \nu_{\mathrm{P}}}\left(v s_{2}+\boldsymbol{v} \cdot \boldsymbol{s}_{2}\right)^{-i v_{\mathrm{P}}} \\
& \quad \times\left\{{ }_{1} F_{1}\left(i v_{\mathrm{T}}, 1, i v x_{2}+i \boldsymbol{v} \cdot \boldsymbol{x}_{2}\right) \nabla_{s_{1}} \varphi_{f}^{*}\left(\boldsymbol{s}_{1}, \boldsymbol{s}_{2}\right) \cdot \nabla_{x_{1} 1} F_{1}\left(i v_{\mathrm{T}}, 1, i v x_{1}+i \boldsymbol{v} \cdot \boldsymbol{x}_{1}\right)\right. \\
& \left.\quad+{ }_{1} F_{1}\left(i v_{\mathrm{T}}, 1, i v x_{1}+i \boldsymbol{v} \cdot \boldsymbol{x}_{1}\right) \nabla_{s_{2}} \varphi_{f}^{*}\left(\boldsymbol{s}_{1}, \boldsymbol{s}_{2}\right) \cdot \nabla_{x_{2} 1} F_{1}\left(i v_{\mathrm{T}}, 1, i v x_{2}+i \boldsymbol{v} \cdot \boldsymbol{x}_{2}\right)\right\}, \\
& \mathcal{B}_{i f}^{(\mathrm{CDW}-\mathrm{EFS}-4 \mathrm{~B})-}=\varphi_{f}^{*}\left(\boldsymbol{s}_{1}, \boldsymbol{s}_{2}\right)\left(v x_{1}+\boldsymbol{v} \cdot \boldsymbol{x}_{1}\right)^{-i v_{\mathrm{T}}}\left(v x_{2}+\boldsymbol{v} \cdot \boldsymbol{x}_{2}\right)^{-i v_{\mathrm{T}}} \\
& \quad \times\left\{{ }_{1} F_{1}\left(i v_{\mathrm{P}}, 1, i v s_{2}+i \boldsymbol{v} \cdot \boldsymbol{s}_{2}\right) \nabla_{x_{1}} \varphi_{i}\left(\boldsymbol{x}_{1}, \boldsymbol{x}_{2}\right) \cdot \nabla_{s_{1} 1} F_{1}\left(i v_{\mathrm{P}}, 1, i v s_{1}+i \boldsymbol{v} \cdot \boldsymbol{s}_{1}\right)\right. \\
& \left.\quad+{ }_{1} F_{1}\left(i v_{\mathrm{P}}, 1, i v s_{1}+i \boldsymbol{v} \cdot \boldsymbol{s}_{1}\right) \nabla_{x_{2}} \varphi_{i}\left(\boldsymbol{x}_{1}, \boldsymbol{x}_{2}\right) \cdot \nabla_{s_{2} 1} F_{1}\left(i v_{\mathrm{P}}, 1, i v s_{2}+i \boldsymbol{v} \cdot \boldsymbol{s}_{2}\right)\right\} \\
& \mathcal{L}_{i f}^{(\mathrm{CDW}-\mathrm{EIS}-4 \mathrm{~B})+}=\mathcal{L}_{i f}^{(\mathrm{CDW}-\mathrm{EFS}-4 \mathrm{~B})-}, \quad \mathcal{L}_{i f}^{(\mathrm{CDW}-\mathrm{EIS}-4 \mathrm{~B}) \pm}=\mathcal{L}_{i f}^{(\mathrm{CDW}-4 \mathrm{~B}) \mp} .
\end{aligned}
$$

Here, $\mathcal{L}_{i f}^{(\mathrm{CDW}-4 \mathrm{~B}) \mp}$ are the $\mathcal{L}$-functions from the CDW-4B method given by Eq. (2.122), where $\mathcal{L}_{i f}^{(\mathrm{CDW}-4 \mathrm{~B})+}=\mathcal{L}_{i f}^{(\mathrm{CDW}-4 \mathrm{~B})-}$. Using (2.123) and (2.124), it follows that the transition amplitudes $T_{i f}^{(\mathrm{CDW}-\mathrm{EIS}-4 \mathrm{~B})+}{ }_{(\boldsymbol{\eta})}$ and $\left.T_{i f}^{(\mathrm{CDW}-\mathrm{EFS}-4 \mathrm{~B})-}{ }^{\boldsymbol{\eta}} \boldsymbol{\eta}\right)$ can be written as:

$$
\begin{gathered}
T_{i f}^{(\mathrm{CDW}-\mathrm{EIS}-4 \mathrm{~B})+}{ }_{(\boldsymbol{\eta})}=-N_{\mathrm{T}}^{2} C_{\mathrm{T}}^{\prime} \iiint \mathrm{d} \boldsymbol{s}_{1} \mathrm{~d} \boldsymbol{s}_{2} \mathrm{~d} \boldsymbol{R} \rho^{2 i \nu_{\mathrm{PT}}} \mathcal{D} \mathcal{B}_{i f}^{(\mathrm{CDW}-\mathrm{EIS}-4 \mathrm{~B})+} \\
T_{i f}^{(\mathrm{CDW}-\mathrm{EFS}-4 \mathrm{~B})-}(\boldsymbol{\eta})=-N_{\mathrm{P}}^{2} C_{\mathrm{P}}^{\prime} \iiint \mathrm{d} \boldsymbol{x}_{1} \mathrm{~d} \boldsymbol{x}_{2} \mathrm{~d} \boldsymbol{R} \rho^{2 i \nu_{\mathrm{PT}}} \mathcal{D} \mathcal{B}_{i f}^{(\mathrm{CDW}-\mathrm{EFS}-4 \mathrm{~B})-} .
\end{gathered}
$$

As in the CDW-4B method, the Coulomb phase $(\mu v \rho)^{2 i \nu_{\mathrm{PT}}}$ is the only remainder from the inter-nuclear potential $V_{\mathrm{PT}}$ in the transition amplitudes $T_{i f}^{(\mathrm{CDW}-\mathrm{EIS}-4 \mathrm{~B})+}(\boldsymbol{\eta})$

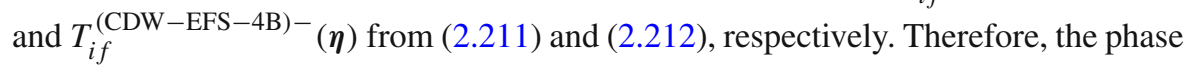
factor $(\mu v \rho)^{2 i \nu_{\mathrm{PT}}}$ disappears from the total cross sections $Q_{i f}^{(\mathrm{CDW}-\mathrm{EIS}-4 \mathrm{~B})+}$ and $Q_{i f}^{(\mathrm{CDW}-\mathrm{EFS}-4 \mathrm{~B})-}$ that become independent of the inter-nuclear potential $V_{\mathrm{PT}}$ :

$$
\begin{aligned}
& Q_{i f}^{(\mathrm{CDW}-\mathrm{EIS}-4 \mathrm{~B})+}\left(a_{0}^{2}\right)=\left.\int \mathrm{d} \boldsymbol{\frac { T _ { i f } ^ { ( \mathrm { CDW } - \mathrm { EIS } - 4 \mathrm { B } ) + } ( \boldsymbol { \eta } ) } { 2 \pi v }}\right|^{2}, \\
& =\int \mathrm{d} \eta\left|\frac{R_{i f}^{(\mathrm{CDW}-\mathrm{EIS}-4 \mathrm{~B})+}(\eta)}{2 \pi v}\right|^{2},
\end{aligned}
$$




$$
\begin{aligned}
Q_{i f}^{(\mathrm{CDW}-\mathrm{EFS}-4 \mathrm{~B})-}\left(a_{0}^{2}\right) & =\int \mathrm{d} \eta\left|\frac{T_{i f}^{(\mathrm{CDW}-\mathrm{EFS}-4 \mathrm{~B})-}(\eta)}{2 \pi v}\right|^{2}, \\
& =\int \mathrm{d} \eta\left|\frac{R_{i f}^{(\mathrm{CDW}-\mathrm{EFS}-4 \mathrm{~B})-}(\eta)}{2 \pi v}\right|^{2},
\end{aligned}
$$

where

$$
\begin{aligned}
& R_{i f}^{(\mathrm{CDW}-\mathrm{EIS}-4 \mathrm{~B})+}(\boldsymbol{\eta})=-N_{\mathrm{T}}^{2} C_{\mathrm{T}}^{\prime} \iiint \mathrm{d} \boldsymbol{s}_{1} \mathrm{~d} \boldsymbol{s}_{2} \mathrm{~d} \boldsymbol{R} \mathcal{D} \mathcal{B}_{i f}^{(\mathrm{CDW}-\mathrm{EIS}-4 \mathrm{~B})+}, \\
& R_{i f}^{(\mathrm{CDW}-\mathrm{EFS}-4 \mathrm{~B})-}{ }_{(\eta)}=-N_{\mathrm{P}}^{2} C_{\mathrm{P}}^{\prime} \iiint \mathrm{d} \boldsymbol{x}_{1} \mathrm{~d} \boldsymbol{x}_{2} \mathrm{~d} \boldsymbol{R} \mathcal{D} \mathcal{B}_{i f}^{(\mathrm{CDW}-\mathrm{EFS}-4 \mathrm{~B})-} .
\end{aligned}
$$

Let us re-emphasize that the superscripts \pm in the transition amplitudes and cross sections for the CDW-EIS-4B and CDW-EFS-4B methods are used to remind us that the former and the latter method are derivable from the post and prior forms of the CDW-4B method, respectively. In other words, the CDW-EIS-4B and CDWEFS-4B methods themselves do not have both the post and prior forms. Rather the CDW-EIS-4B method exists only in the post variant, $T_{i f}^{(\mathrm{CDW}-\mathrm{EIS}-4 \mathrm{~B})+}(\boldsymbol{\eta})$, whereas the CDW-EFS-4B method is defined solely in the prior version, $T_{i f}^{(\mathrm{CDW}-\mathrm{EFS}-4 \mathrm{~B})-}(\boldsymbol{\eta})$.

\subsubsection{The link between the prior/post BDW-4B and CDW-EFS/EIS-4B methods}

Here, we establish the relationships between the post/prior BDW-4B and CDWEIS/EFS-4B methods. We do that by juxtaposing the transition amplitudes in these methods so as to exhibit their similarities. To this end, we cast Eqs. (2.198) and (2.212) into the following forms:

$$
\left.\begin{array}{c}
T_{i f}^{(\mathrm{BDW}-4 \mathrm{~B})-}(\boldsymbol{\eta})=-N_{\mathrm{P}}^{2} C_{\mathrm{P}}^{\prime} \iiint \mathrm{d} \boldsymbol{x}_{1} \mathrm{~d} \boldsymbol{x}_{2} \mathrm{~d} \boldsymbol{R}(v R+\boldsymbol{v} \cdot \boldsymbol{R})^{-2 i v_{\mathrm{T}}} \mathcal{D} \mathcal{H}_{i f}^{-} \\
T_{i f}^{(\mathrm{CDW}-\mathrm{EFS}-4 \mathrm{~B})-}(\boldsymbol{\eta})=-N_{\mathrm{P}}^{2} C_{\mathrm{P}}^{\prime} \iiint \mathrm{d} \boldsymbol{x}_{1} \mathrm{~d} \boldsymbol{x}_{2} \mathrm{~d} \boldsymbol{R} \prod_{k=1}^{2}\left(v x_{k}+\boldsymbol{v} \cdot \boldsymbol{x}_{k}\right)^{-i v_{\mathrm{T}}} \mathcal{D} \mathcal{H}_{i f}^{-}
\end{array}\right\},
$$

$$
\begin{aligned}
\mathcal{H}_{i f}^{-}= & (\mu v \rho)^{2 i v_{\mathrm{PT}}} \varphi_{f}^{*}\left(\boldsymbol{s}_{1}, \boldsymbol{s}_{2}\right) \\
& \times\left\{{ }_{1} F_{1}\left(i v_{\mathrm{P}}, 1, i v s_{2}+i \boldsymbol{v} \cdot \boldsymbol{s}_{2}\right) \nabla_{x_{1}} \varphi_{i}\left(\boldsymbol{x}_{1}, \boldsymbol{x}_{2}\right) \cdot \nabla_{s_{1} 1} F_{1}\left(i v_{\mathrm{P}}, 1, i v s_{1}+i \boldsymbol{v} \cdot \boldsymbol{s}_{1}\right)\right. \\
& \left.+{ }_{1} F_{1}\left(i v_{\mathrm{P}}, 1, i v s_{1}+i \boldsymbol{v} \cdot \boldsymbol{s}_{1}\right) \nabla_{x_{2}} \varphi_{i}\left(\boldsymbol{x}_{1}, \boldsymbol{x}_{2}\right) \cdot \nabla_{s_{2} 1} F_{1}\left(i v_{\mathrm{P}}, 1, i v s_{2}+i \boldsymbol{v} \cdot \boldsymbol{s}_{2}\right)\right\}
\end{aligned}
$$

Here, the gradient-gradient operators for the distorting potentials are the same in both methods. However, there are two unequal terms $(v R+\boldsymbol{v} \cdot \boldsymbol{R})^{-2 i v_{\mathrm{T}}}$ and $\left[\left(v x_{1}+\boldsymbol{v} \cdot \boldsymbol{x}_{1}\right)\left(v x_{2}+\boldsymbol{v} \cdot \boldsymbol{x}_{2}\right)\right]^{-i v_{\mathrm{T}}}$ in the prior BDW-4B and CDW-EFS-4B methods, 
respectively. This is the only difference between these two methods. Such a difference is negligible only in the asymptotic region of the exit channel since according to (2.108), we have:

$$
\left[\left(v x_{1}+\boldsymbol{v} \cdot \boldsymbol{x}_{1}\right)\left(v x_{2}+\boldsymbol{v} \cdot \boldsymbol{x}_{2}\right)\right]^{-i v_{\mathrm{T}}} \underset{x_{1}, \tilde{x}_{2} \rightarrow \infty}{\approx}(v R+\boldsymbol{v} \cdot \boldsymbol{R})^{-2 i v_{\mathrm{T}}} .
$$

Of course, the integrals in (2.219) are over all the distances $\left\{\boldsymbol{x}_{1}, \boldsymbol{x}_{2}, \boldsymbol{R}\right\}$ and not just over their radial asymptotic values. It is this circumstance that makes the prior BDW4B and CDW-EFS-4B methods differ from each other.

A similar situation is also encountered when comparing the post BDW-4B and CDW-EIS-4B methods. This is at once seen by writing together the transition amplitudes (2.199) and (2.211) as:

$$
\begin{aligned}
& T_{i f}^{(\mathrm{BDW}-4 \mathrm{~B})+}{ }_{(\boldsymbol{\eta})}{ }^{(\mathrm{B})}-N_{\mathrm{T}}^{2} C_{\mathrm{T}}^{\prime} \iiint \mathrm{d} \boldsymbol{s}_{1} \mathrm{~d} \boldsymbol{s}_{2} \mathrm{~d} \boldsymbol{R}(v \boldsymbol{R}-\boldsymbol{v} \cdot \boldsymbol{R})^{-2 i \nu_{\mathrm{P}}} \mathcal{D} \mathcal{H}_{i f}^{+} \\
& \left.T_{i f}^{(\mathrm{CDW}-\mathrm{EIS}-4 \mathrm{~B})+}(\boldsymbol{\eta})=-N_{\mathrm{T}}^{2} C_{\mathrm{T}}^{\prime} \iiint \mathrm{d} \boldsymbol{s}_{1} \mathrm{~d} \boldsymbol{s}_{2} \mathrm{~d} \boldsymbol{R} \prod_{k=1}^{2}\left(v s_{k}+\boldsymbol{v} \cdot \boldsymbol{s}_{k}\right)^{-i \nu_{\mathrm{P}}} \mathcal{D} \mathcal{H}_{i f}^{+}\right\}_{(2.22)}, \\
& \mathcal{H}_{i f}^{+}=(\mu v \rho)^{2 i \nu_{\mathrm{PT}}} \varphi_{i}\left(\boldsymbol{x}_{1}, \boldsymbol{x}_{2}\right) \\
& \quad \times\left\{{ }_{1} F_{1}\left(i v_{\mathrm{T}}, 1, i v x_{2}+i \boldsymbol{v} \cdot \boldsymbol{x}_{2}\right) \nabla_{s_{1}} \varphi_{f}^{*}\left(\boldsymbol{s}_{1}, \boldsymbol{s}_{2}\right) \cdot \nabla_{x_{1} 1} F_{1}\left(i v_{\mathrm{T}}, 1, i v x_{1}+i \boldsymbol{v} \cdot \boldsymbol{x}_{1}\right)\right. \\
& \left.\quad+{ }_{1} F_{1}\left(i \nu_{\mathrm{T}}, 1, i v x_{1}+i \boldsymbol{v} \cdot \boldsymbol{x}_{1}\right) \nabla_{s_{2}} \varphi_{f}^{*}\left(\boldsymbol{s}_{1}, \boldsymbol{s}_{2}\right) \cdot \nabla_{x_{2} 1} F_{1}\left(i \nu_{\mathrm{T}}, 1, i v x_{2}+i \boldsymbol{v} \cdot \boldsymbol{x}_{2}\right)\right\} .
\end{aligned}
$$

Here too, both methods possess the identical gradient-gradient potential operators. Further, we see that the sole difference between the post BDW-4B and CDW-EIS-4B methods is the terms $(v R-\boldsymbol{v} \cdot \boldsymbol{R})^{-2 i v_{\mathrm{P}}}$ and $\left[\left(v s_{1}+\boldsymbol{v} \cdot \boldsymbol{s}_{1}\right)\left(v s_{2}+\boldsymbol{v} \cdot \boldsymbol{s}_{2}\right)\right]^{-i \nu_{\mathrm{P}}}$ from the former and the latter approximation. Such a difference is small only in the asymptotic region of the entrance channel as per (2.107) which implies:

$$
\left[\left(v s_{1}+\boldsymbol{v} \cdot \boldsymbol{s}_{1}\right)\left(v s_{2}+\boldsymbol{v} \cdot \boldsymbol{s}_{2}\right)\right]_{s_{1}, s_{2} \rightarrow \infty}^{\approx \nu_{\mathrm{P}}}(v R-\boldsymbol{v} \cdot \boldsymbol{R})^{-2 i \nu_{\mathrm{P}}}
$$

However, the integrals in (2.222) cover all the distances $\left\{\boldsymbol{s}_{1}, \boldsymbol{s}_{2}, \boldsymbol{R}\right\}$ and not just their asymptotically large values. This fact is responsible for any difference found in the computations by means of the CDW-EIS-4B and the post BDW-4B methods.

The initial and final heliumlike bound-state wave functions $\varphi_{i}\left(\boldsymbol{x}_{1}, \boldsymbol{x}_{2}\right)$ and $\varphi_{f}\left(s_{1}, s_{2}\right)$ decay fast (exponentially) with the increasing values of $\left\{x_{1}, x_{2}\right\}$ and $\left\{s_{1}, s_{2}\right\}$, respectively. This is readily apparent from e.g. the heliumlike ground state wave functions with either one [125] (Hylleraas) or four variational parameters [126-128] (Löwdin, Green et al, Silverman et al): 


$$
\left.\begin{array}{ll}
\varphi_{i}\left(\boldsymbol{x}_{1}, \boldsymbol{x}_{2}\right)=\frac{\left(Z_{\mathrm{T}}^{\mathrm{eff}}\right)^{3}}{\pi} \mathrm{e}^{-Z_{\mathrm{T}}^{\text {eff }}\left(x_{1}+x_{2}\right)}, & Z_{\mathrm{T}}^{\text {eff }}=Z_{\mathrm{T}}-\frac{5}{16} \\
\varphi_{f}\left(\boldsymbol{s}_{1}, \boldsymbol{s}_{2}\right)=\frac{\left(Z_{\mathrm{P}}^{\mathrm{eff}}\right)^{3}}{\pi} \mathrm{e}^{-Z_{\mathrm{P}}^{\text {eff }}\left(s_{1}+s_{2}\right)}, & Z_{\mathrm{P}}^{\text {eff }}=Z_{\mathrm{P}}-\frac{5}{16}
\end{array}\right\}, \text { Hylleraas [125]. }
$$

Therefore, it is the small values of $\left\{x_{1}, x_{2}\right\}$ and $\left\{s_{1}, s_{2}\right\}$ that provide the dominant contributions to the integrals in (2.219) and (2.222). Moreover, in the regions of the small values of $\left\{x_{1}, x_{2}\right\}$ and $\left\{s_{1}, s_{2}\right\}$, the behaviors of $\prod_{k=1}^{2}\left(v x_{k}+\boldsymbol{v} \cdot \boldsymbol{x}_{k}\right)^{-i v_{\mathrm{T}}}$ and $(v R-\boldsymbol{v} \cdot \boldsymbol{R})^{-2 i \nu_{\mathrm{T}}}$ in (2.219) as well as of $\prod_{k=1}^{2}\left(v s_{k}+\boldsymbol{v} \cdot \boldsymbol{s}_{k}\right)^{-i \nu_{\mathrm{P}}}$ and $(v R+\boldsymbol{v} \cdot \boldsymbol{R})^{-2 i v_{\mathrm{P}}}$ in (2.222) are very different. This is prone to yield the significant discrepancies in the cross sections computed by means of the CDW-EFS-4B and the prior BDW-4B methods, as well as between the CDW-EIS-4B and the post BDW-4B methods.

\subsubsection{The link between the prior/post CDW-4B and CDW-EFS/EIS-4B methods}

Continuing with the preceding lines, it is also instructive to juxtapose the CDWEFS/EIS-4B and the prior/post CDW-4B methods to directly see their similarities and differences. Thus, putting together Eqs. (2.126) and (2.212), we have:

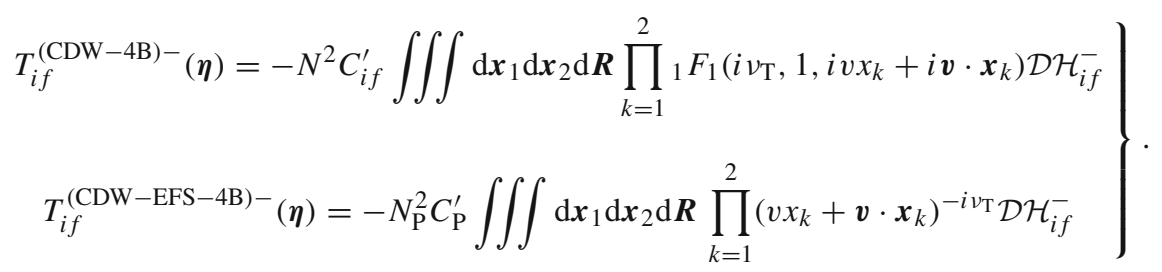

The same gradient-gradient potential operator is present in these two transition amplitudes. Otherwise, the CDW-EFS-4B method is seen to be an approximation to the post CDW-4B method. It replaces the product of the two full confluent hypergeometric functions $N_{\mathrm{T}}^{2} \prod_{k=11}^{2} F_{1}\left(i \nu_{\mathrm{T}}, 1, i v x_{k}+i \boldsymbol{v} \cdot \boldsymbol{x}_{k}\right)$ for the electrons $e_{1,2}$ by their asymptotic forms given by the Coulomb logarithmic phases $\prod_{k=1}^{2}\left(v x_{k}+\boldsymbol{v} \cdot \boldsymbol{x}_{k}\right)^{-i v_{\mathrm{T}}}$ as per (2.104). This replacement is valid only outside the integrals for the transition amplitudes and exclusively at very large distances $\left(x_{1} \rightarrow \infty, x_{2} \rightarrow \infty\right)$. Since the capture cross sections are dominated by the small values of $\left\{x_{1}, x_{2}\right\}$, at which the said replacement breaks down, the CDW-EFS-4B and prior CDW-4B method are expected to yield different results, especially at lower part of intermediate incident velocities.

In the same vein, we can collect Eqs. (2.127) and (2.211) for a straightforward comparison: 


$$
\left.\begin{array}{c}
T_{i f}^{(\mathrm{CDW}-4 \mathrm{~B})+}(\boldsymbol{\eta})=-N^{2} C_{i f}^{\prime} \iiint \mathrm{d} \boldsymbol{s}_{1} \mathrm{~d} \boldsymbol{s}_{2} \mathrm{~d} \boldsymbol{R} \prod_{k=1}^{2}{ }_{1} F_{1}\left(i v_{\mathrm{P}}, 1, i v s_{k}+i \boldsymbol{v} \cdot \boldsymbol{s}_{k}\right) \mathcal{D} \mathcal{H}_{i f}^{+} \\
T_{i f}^{(\mathrm{CDW}-\mathrm{EIS}-4 \mathrm{~B})+}{ }_{(\boldsymbol{\eta})}=-N_{\mathrm{T}}^{2} C_{\mathrm{T}}^{\prime} \iiint \mathrm{d} \boldsymbol{s}_{1} \mathrm{~d} \boldsymbol{s}_{2} \mathrm{~d} \boldsymbol{R} \prod_{k=1}^{2}\left(v s_{k}+\boldsymbol{v} \cdot \boldsymbol{s}_{k}\right)^{-i \nu_{\mathrm{P}}} \mathcal{D} \mathcal{H}_{i f}^{+}
\end{array}\right\}
$$

These two expressions share the common gradient-gradient potential operator. According to (2.103), (2.206) and (2.208), the CDW-EIS-4B method is observed to make an additional approximation to the post CDW-4B method using the Coulomb logarithmic phases $\prod_{k=1}^{2}\left(v s_{k}+\boldsymbol{v} \cdot \boldsymbol{s}_{k}\right)^{-i \nu_{\mathrm{P}}}$ instead of the full confluent hypergeometric functions $N_{\mathrm{P}}^{2} \prod_{k=11}^{2} F_{1}\left(i \nu_{\mathrm{P}}, 1, i v s_{k}+i v \cdot s_{k}\right)$ for the electrons $e_{1}$ and $e_{2}$. Such an approximation is justified solely at simultaneously large distances $s_{1} \rightarrow \infty$ and $s_{2} \rightarrow \infty$, but fails at small values of $\left\{s_{1}, s_{2}\right\}$ that otherwise provide the dominant contribution to the cross sections. The errors invoked by using the asymptotic Coulomb phases instead of the confluent confluent hypergeometric functions for the electrons are expected to increase with decreasing incident velocity.

\section{Convergence issues with the Born series for rearrangement collisions}

Aaron et al. [129] pointed out that the Born series for the transition operators diverges for three-body rearrangement collisions. However, neither the transition operators nor the related total scattering wave functions are observable (physically measurable quantities). What actually matters is the status of convergence of certain observables of the main interest, e.g. scalar products that contain the transition operators and total scattering wave functions. In scalar products, such as those from cross sections, the transition operators and total scattering wave functions are embedded in integrals over all the configuration and/or momentum space. This circumstance may well wash out the pathological/divergent features of the transition operators and total scattering wave functions within the transition amplitudes. Indeed, it has been demonstrated by Corbett [130] that for a divergent $T$-operator series, convergence can nevertheless exist for both the series of total scattering wave function and the $T$-matrix elements. This means that the conditions in the Born series for the $T$-operator convergence are more restrictive than those for the total wave functions. It also implies that the convergence conditions in the Born series for the total scattering wave functions are more restrictive that those for the $T$-matrix elements. An appropriate illustration of this important conclusion has been reported by Dettman and Leibfried [131] for a special case of rearrangement collisions with the $\delta$-function interactions. For this particular scattering, it has been found [131] that despite the existing operator divergence, the resulting physical transition amplitude is convergent.

Dodd and Greider $[132,133]$ have studied the convergence features of the distorted wave Born series for three-particle rearrangement collisions with short-range interac- 
tions. They analyzed the case of two heavy and one light particles. We shall briefly discuss their concept adopted to atomic collisions for single electron capture by nuclei from hydrogenlike atomic systems:

$$
Z_{\mathrm{P}}+\left(Z_{\mathrm{T}}, e\right)_{i} \longrightarrow\left(Z_{\mathrm{P}}, e\right)_{f}+Z_{\mathrm{T}}
$$

Here, as before, both the projectile and target nucleus of charges $Z_{\mathrm{P}}$ and $Z_{\mathrm{T}}$ are heavy particles. The main focus in Refs. [132,133] was upon the so called "disconnected diagrams" because these lead to divergence of the Born series. The disconnected diagrams are those Feynman diagrams that describe the intermediate steps of collision (3.1) with two particles interacting with each other, while the third particle is propagating freely. For example, the typical kernel $U_{f}^{\dagger} G_{0}^{+} U_{i}$, in terms of the total three-body free-particle Green operator $G_{0}^{+}$, would become disconnected if a potential from e.g. $U_{f}^{\dagger}$ were also contained in $U_{i}$. Therefore, in order to have only connected diagrams that, in turn, yield the divergence-free operator Born series, it suffices to modify the kernel of the series in such a way that no potential from e.g. $U_{i}$ would be repeated in $U_{f}^{\dagger}$. This can be achieved by introducing a virtual channel $x$ with a model potential $V_{x}$ (real- or complex-valued) and the associated Green operators $g_{x}^{ \pm}$defined by:

$$
g_{x}^{ \pm}=\frac{1}{E-H+V_{x} \pm i \varepsilon}
$$

where $E$ and $H$ are the total energy and the total Hamiltonian of three particles encountered in process (3.1) for which $U_{i, f}$ are the perturbation distorting potentials. To proceed, Dodd and Greider [133] used the three coupled Faddeev equations [134137] for the transition amplitude of the studied three-body problem. Then they exploit the suitable mass ratios of the two heavy and one light particle to reduce the three to two coupled Faddeev equations and finally arrive at the full transition amplitude whose kernel can be connected by making a suitable choice of potential $V_{x}$. The see this, we first note that there is a direct relationship between the model $g_{x}^{+}$and the total Green operator $G^{+}$as:

$$
G^{+}=g_{x}^{+}\left(1+V_{x} G^{+}\right), \quad G^{+}=\frac{1}{E-H+i \varepsilon} .
$$

Inserting (3.3) into (2.74), where $U_{i, f}, G^{+}$and $\chi_{i, f}^{ \pm}$refer to process (3.1), we obtain the modified full transition amplitudes in e.g. the prior form which we write here together with its original counterpart, to enable a direct comparison:

$$
\left.\begin{array}{c}
T_{i f}^{-} \text {(Modified) }=\left\langle\chi_{f}^{-}\left|\left\{1+U_{f} g_{x}^{-}\left(1+V_{x} G^{-}\right)\right\}^{\dagger} U_{i}\right| \chi_{i}^{-}\right\rangle \\
T_{i f}^{-}(\text {Original })=\left\langle\chi_{f}^{-}\left|\left(1+U_{f} G^{-}\right)^{\dagger} U_{i}\right| \chi_{i}^{-}\right\rangle
\end{array}\right\} .
$$

As a check, for $V_{x}=0$, it follows from (3.3) that $g_{x}^{+}=G^{+}$, in which case we have $T_{i f}^{-}($Modified $)=T_{i f}^{-}($Original $)$, as it should be. The Born series of the new transition 
amplitude $T_{i f}^{-}$(Modified) will be void of disconnected diagrams if e.g. no part in the chosen potential $V_{x}$ is repeated in $U_{f}$.

By setting $G^{+}=0$ in (3.4), one obtains the first-order approximations $T_{i f}^{(1)-}$ (Modified) and $T_{i f}^{(1)-}$ (Original) to $T_{i f}^{-}$(Modified) and $T_{i f}^{-}$(Original), respectively:

$$
\left.\begin{array}{c}
T_{i f}^{(1)-}(\text { Modified })=\left\langle\chi_{f}^{-}\left|\left(1+U_{f} g_{x}^{-}\right)^{\dagger} U_{i}\right| \chi_{i}^{-}\right\rangle \\
T_{i f}^{(1)-}(\text { Original })=\left\langle\chi_{f}^{-}\left|U_{i}\right| \chi_{i}^{-}\right\rangle
\end{array}\right\}
$$

As stated, a judicious choice of $V_{x}$ leads to a divergence-free modified Born series for the transition amplitude $T_{i f}^{-}$(Modified). This guarantees that the first-order contribution $T_{i f}^{(1)-}$ (Modified) in that series is a meaningful lowest order term to a divergence-free perturbation expansion generated from $T_{i f}^{-}$(Modified). However, the price to pay for this achievement is that $T_{i f}^{(1)-}$ (Modified) is more complicated than $T_{i f}^{(1)-}$ (Original) since the modified $T$-matrix element contains an additional propagator $U_{f} g_{x}^{-}$. Nevertheless, with a particular choice of $V_{x}$, satisfying the mentioned Dodd-Greider constraint, the same analytical result for the transition amplitude $T_{i f}^{(1)-}$ (Original) from the CDW-3B method [138] in the case $Z_{\mathrm{P}}=Z_{\mathrm{T}}=1$ for process (3.1) have also been obtained in Ref. [139] using $T_{i f}^{(1)-}$ (Modified). A similar situation is encountered in the BCIS-3B, BDW-3B and CDW-EIS/EFS-3B methods, as well.

In practice, besides having a divergence-free Born series, its first few successive terms should also be computed numerically to see their behaviors regarding the smoothness and convergence rate. Such an insight could help to empirically assess the possibility for convergence of the entire Born series. This has been the subject of a number of studies where the exact numerical computations were carried out in the 1st [140-144], 2nd [145-149] and 3rd [150] Born approximations to the series for the full $T$-matrix elements with $Z_{\mathrm{P}}=Z_{\mathrm{T}}=1$ in process (3.1). The outcome is that all the three Born approximations are well-behaved, smooth functions at all impact energies and for any scattering angle. Further, these studies show that, at high impact energies (in the $\mathrm{MeV}$ region), the 2nd Born approximation dominates over both the 1 st and the 3rd Born approximations. This steady trend, especially with the recent availability of the exact cross sections for the 3rd Born approximation [150], is an improved assessment of the convergence rate of the Born series for rearrangement collisions of the prototype (3.1).

\section{Illustrations}

In the preceding exposition, we illuminated the similarities and differences among various distorted wave models for the general case of the arbitrary nuclear charges $Z_{\mathrm{P}}$ and $Z_{\mathrm{T}}$ in process (2.1) for double electron capture. For example, in sub-sections 2.5.6 and 
2.5.7, we highlighted the relationships among the post/prior BDW-4B and CDW-EFSEIS methods as well as among the prior/post CDW-4B and CDW-EFS/EIS methods, respectively. Moreover, throughout the analysis, we emphasized the role of the double continuum intermediate states populated by the two electrons prior to their capture by the projectiles. Such twofold electronic ionization continua are explicitly included in the second-order methods, either in one channel (BCIS-4B, BDW-4B) or two channels (CDW-4B, CDW-EIS/EFS-4B). The first-order theories, such as the CB14B method, do not take into account the double electronic continuum intermediate states since the initial and final total scattering states are considered to be the dressed channel states $\Phi_{i, f}^{ \pm}$. The states $\Phi_{i, f}^{ \pm}$are the products of the unperturbed channel states $\Phi_{i, f}$ and the Coulomb logarithmic phase factors exp $\left( \pm i v_{i, f} \ln (v R \mp \boldsymbol{v} \cdot \boldsymbol{R})\right)$ generated by the asymptotically present tails of the residual Coulomb potentials, $V_{i, f}^{\infty}=Z_{\mathrm{P}, \mathrm{T}}\left(Z_{\mathrm{T}, \mathrm{P}}-2\right) / R$. Here, $\boldsymbol{R}$ is the vector of the inter-nuclear axis $R$. Simultaneously, $\boldsymbol{R}$ is also the difference between the electronic position vectors $\left\{\boldsymbol{x}_{k}, \boldsymbol{s}_{k}\right\}$ relative to $Z_{\mathrm{T}}$ and $Z_{\mathrm{P}}$, respectively, $\boldsymbol{R}=\boldsymbol{x}_{k}-\boldsymbol{s}_{k}(k=1,2)$. In other words, using $\boldsymbol{R}$ in the Coulomb logarithmic phases for the BCIS-4B and BDW-methods amounts to correlating the two Coulomb centers (the nuclei of the projectile and of the target). By contrast, the Coulomb logarithmic phases in terms of $\boldsymbol{s}_{k}$ (CDW-EIS-4B) or $\boldsymbol{x}_{k}$ (CDW-EFS-4B) deal only with the projectile or the target Coulomb center at a time (hence no correlation between the two centers). This difference is immaterial only at large $\left\{R, s_{k}\right\}$ (entrance channel) and large $\left\{R, x_{k}\right\}$ (exit channel), according to (2.113) and (2.114), but becomes essential at finite distances when these Coulomb logarithmic phases are employed in the integrals over all distances in the matrix elements of the transition amplitudes from the BCIS-4B, BDW-4B and CDW-EIS/EFS-4B methods.

These features are illustrated in Fig. 1 where the total cross sections from all the analyzed methods (CB1-4B, CDW-4B, CDW-EIS/EFS-4B, BCIS-4B, BDW-4B) are compared with the existing experimental data for double electron capture from helium by alpha particles. Here, the theories refer to the ground-to-ground state transition alone:

$$
{ }^{4} \mathrm{He}^{2+}+{ }^{4} \mathrm{He}\left(1 \mathrm{~s}^{2}\right) \longrightarrow{ }^{4} \mathrm{He}\left(1 \mathrm{~s}^{2}\right)+{ }^{4} \mathrm{He}^{2+}
$$

On the other hand, the measurements displayed on Fig. 1 are for all the final bound states (ground and excited) of helium as symbolized by:

$$
{ }^{4} \mathrm{He}^{2+}+{ }^{4} \mathrm{He}\left(1 \mathrm{~s}^{2}\right) \longrightarrow{ }^{4} \mathrm{He}(\Sigma)+{ }^{4} \mathrm{He}^{2+} .
$$

Nevertheless, it still makes sense to compare theories for (4.1) and experiments for (4.2) in Fig. 1, since it has been shown in the CDW-4B method [45] that at least above $1000 \mathrm{keV}$ the sum of the contributions from the singly and doubly excited final states of helium is small. However, it would be important to assess the contribution from the excited states also below $1000 \mathrm{keV}$. The theoretical results shown in Fig. 1 are all obtained using (2.225) as the one-parameter Hylleraas wave function [125] for both the initial and final ground states of helium in symmetric double charge exchange (4.1) with $Z_{\mathrm{K}}^{\text {eff }}=2-0.3125=1.6875(\mathrm{~K}=\mathrm{P}, \mathrm{T})$. Using the heliumlike groundstate wave functions with one parameter [125] (Hylleraas) and four parameters [126- 
Striking Role of Intermediate Ionization for Two-Electron Transfer in Ion-Atom Collisions

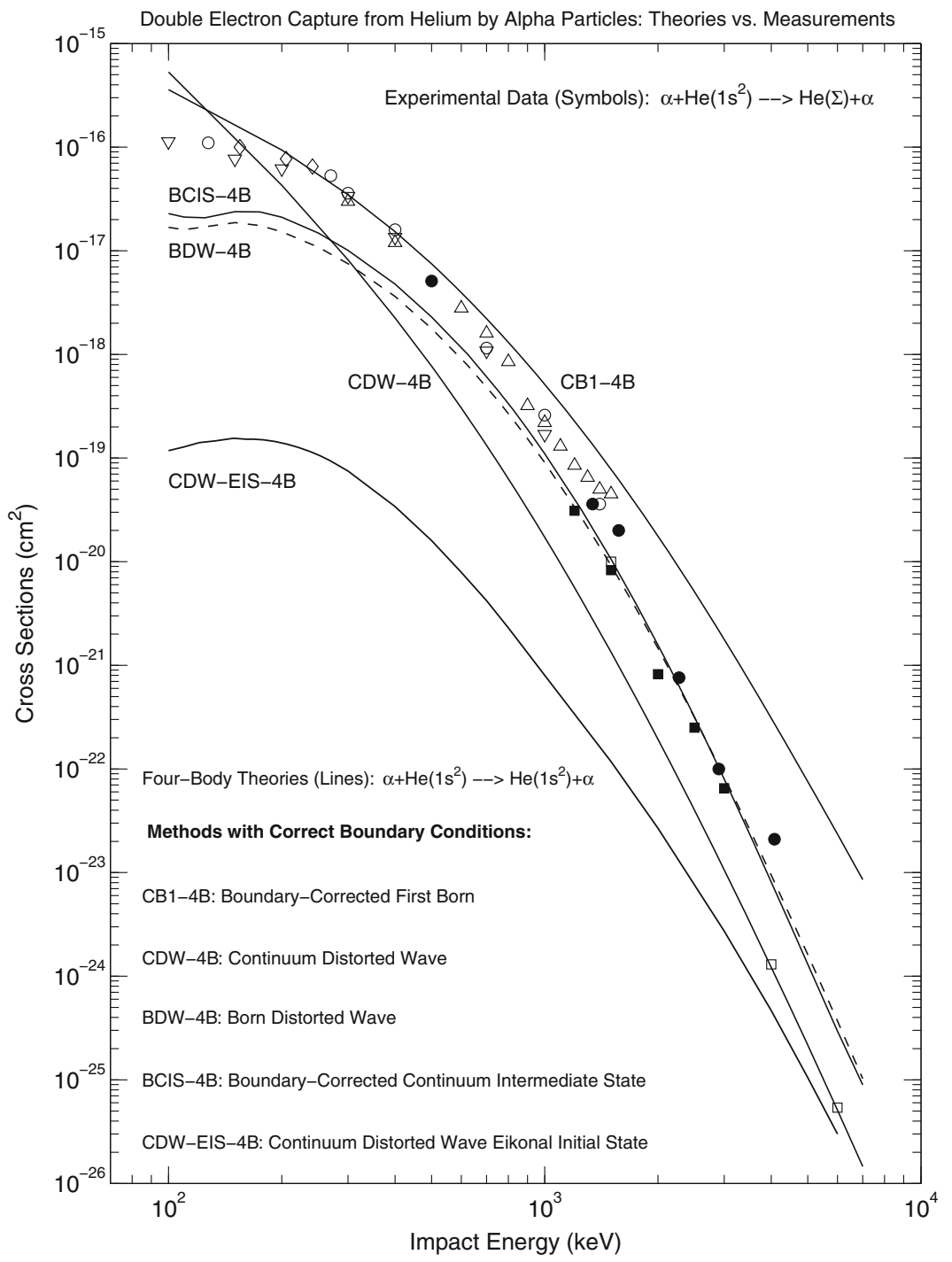

Fig. 1 Total cross sections $Q\left(\mathrm{~cm}^{2}\right)$ as a function of the incident energy $E(\mathrm{keV})$ for processes (4.1) and (4.2): double electron capture from ${ }^{4} \mathrm{He}\left(1 s^{2}\right)$ by ${ }^{4} \mathrm{He}^{2+}$. The results of the computations for process (4.1), i.e. ${ }^{4} \mathrm{He}^{2+}+{ }^{4} \mathrm{He}\left(1 s^{2}\right) \rightarrow{ }^{4} \mathrm{He}\left(1 s^{2}\right)+{ }^{4} \mathrm{He}^{2+}$ (ground-to-ground-bound-state transition with the initial and final one-parameter helium wave functions of Hylleraas [125]) by using 5 different methods whose acronyms are indicated near the lines: CB1-4B [1,3,33,34], BCIS-4B [1,3,32], BDW-4B [1,3,41], CDW-4B [1,3,30,42] and CDW-EIS-4B [47]. Experimental data for process (4.2), i.e. ${ }^{4} \mathrm{He}^{2+}+{ }^{4} \mathrm{He}\left(1 s^{2}\right) \rightarrow{ }^{4} \mathrm{He}(\Sigma)+{ }^{4} \mathrm{He}^{2+}$ (ground-to-any-bound-state transitions): $\Delta$ [58], $\diamond[61], \circ[69], \nabla$ [75], $\mathbf{\square}$ [76], $\square$ [79] and • [80]. For computational details, see the cited theoretical studies. In particular, the lines for the BCIS-4B and BDW-4B methods show here the $\mathrm{Q}$ data obtained by 5-dimensional numerical quadratures using the Gauss-Legendre rule with 96 points and weights per each integration axis [32] 
128] within the CDW-4B method at energies $100-7000 \mathrm{keV}$, it has been verified in Refs. [32,42], that the total cross sections are not overly sensitive to the static interelectronic correlations. It would be useful to check whether this conclusion regarding (4.1) also holds true for the BCIS-4B and BDW-4B methods.

The status of the first-order theories is evident from Fig. 1 when comparing the CB14B method with the experimental data $[58,61,69,75,76,79,80]$ (presently taken from the most exhaustive tabulated database Refs. [85,86]). In Fig. 1, the cross sections from the CB1-4B method are seen to closely follow the measurements at impact energies from 200 to $800 \mathrm{keV}$. However, the discrepancy between the CB1-4B method and experiments keeps on increasing with the augmented impact energy. Thus, e.g. at $3000 \mathrm{keV}$, the CB1-4B method overestimates the experimental data by more than an order of magnitude. This significant and systematic overestimation is a clear indication of the importance of the double electronic continuum intermediate states that are not taken into account in the CB1-4B method.

In order to have a more quantitative insight into the role of the intermediate double ionization continua of the two electrons, it is necessary to pass onto comparisons of measurements with the second-order methods. To achieve this goal, we examine the overall performance of the CDW-4B and CDW-EIS-4B methods. Thus, it is seen in Fig. 1 that the cross sections from the CDW-EIS-4B method are strongly suppressed relative to those of the CDW-4B method. For example, at the impact energy of $100 \mathrm{keV}$, the cross sections of the CDW-EIS-4B method underestimate the results of the CDW4B method by nearly four orders of magnitude. Such a gigantic and unprecedented discrepancy is still huge at larger energies. For instance, even at $1000 \mathrm{keV}$, the predictions by the CDW-EIS-4B method are an order of magnitude lower than those due to the CDW-4B method. The magnitudes of the cross sections from the CDW-4B method are seen in Fig. 1 to significantly underestimate the experimental data at impact energies 200-3000 keV. Also the lineshapes (the behaviors of the cross sections as a function of the impact energy) are different in the CDW-4B method and measurements. At still higher energies (4000 and $7000 \mathrm{keV}$ ), the CDW-4B method is in excellent agreement with the measurement from Ref. [79]. However, particularly at $4000 \mathrm{keV}$, the situation is inconclusive since the cross sections from the two independent measurements $[79,80]$ differ by more than an order of magnitude (see the pertinent remark on p. 3837 in Ref. [32]).

Further, it is observed in Fig. 1 that the cross sections from the BCIS-4B and the BDW-4B methods agree quite well with each other concerning both their magnitudes and lineshapes. The cross sections from these two methods would be identical if the helium bound state wave function were exact. For example, the same cross sections are obtained from the BCIS-3B and BDW-3B methods in the case of electron capture from hydrogenlike atomic systems by nuclei in process (3.1) for any initial and final state. In Fig. 1, at intermediate-to-high energies $100-3000 \mathrm{keV}$, the curvatures of the cross sections from the BCIS-3B and BDW-3B methods are similar to the common lineshape that could be drawn through the depicted experimental data to guide the eye. As to the magnitudes of the cross sections, the BCIS-4B and BDW-4B methods underestimate the experimental data below $1000 \mathrm{keV}$, albeit by a much smaller factor than in the CDW4B method. On the other hand, the BCIS-4B and BDW-4B methods are in excellent agreement with the experimental data at impact energies $1000-3000 \mathrm{keV}$. However, 
no similar validity assessment is possible at still higher energies $(4000,7000 \mathrm{keV})$ because of the mentioned more than an order of magnitude discrepancy between the two measurements reported in Refs. [79,80]. In fact, at $4000 \mathrm{keV}$, the cross sections from the BCIS-4B and BDW-4B methods are near the mid-point between the two symbols for experimental data from Refs. [79,80]. Needless to say, it would be highly desirable and important to perform some new measurements on (4.2) so as to clarify this unusual disagreement between the two independent measurements of Schuch et al. [79] and Afrosimov et al. [80].

Evidently, there is no post-prior discrepancy for (4.1) and (4.2) because these are symmetric double capture events in collisions between alpha particles and helium. Moreover, in the case of general heavy particle collisions $\left(m_{\mathrm{P}, \mathrm{T}} \gg 1\right)$, and not just single or double capture processes, the numerical values of total cross sections are expected to be the same for computations with either the logarithmic Coulomb phase factors or the associated full Coulomb wave functions for the relative motion of the two heavy scattering aggregates. This has explicitly been confirmed in the CB1-4B as well as in the BCIS-3B and BCIS-4B methods for single electron capture in heavy particle collisions [151,152]. The same conclusion is anticipated to apply also to double capture. Surprisingly, however, the total cross sections in e.g. the four-body boundary-corrected continuum intermediate state method for double electron capture from heliumlike atomic systems by heavy nuclei are significantly different (especially at intermediate-to-lower impact energies) when computed with the full Coulomb wave function [49-53] and with its logarithmic Coulomb phase [1,3,32]. To clarify the matter, especially regarding an earlier observation [3] made on this discrepancy, we performed a new thorough computation the results of which will be published shortly.

\section{Discussion and conclusions}

In this work, a parallel structure presentation is expounded by juxtaposing the conventional and distorted wave formalisms of the general quantum-mechanical scattering theory for double electron capture from heliumlike atomic systems by heavy nuclei. The former deals directly with the original, analytically unsolvable collisional problem. The latter solves (by analytical means) a flexible model problem with certain judicious choices of the distorting potentials and the corresponding distorted wave functions.

Crucially, however, both frameworks for the full transition amplitudes (as well as for any approximation) in the prior and post forms satisfy the correct boundary conditions. These conditions are formulated by the following three simultaneous requirements for:

- (I) the proper asymptotic behaviors of the total scattering wave functions in the initial and final states of the entire system,

- (II) the consistent connections between the perturbation potentials and the total scattering wave functions in the entrance and exit channels, and

- (III) the presence of short-range perturbation potentials in the transition amplitudes. 
The most appropriate way to achieve this in practice runs as follows. Whenever there are residual Coulomb potentials in the asymptotic regions of the considered collision:

- (i) the unperturbed channel states have to be multiplied by the corresponding logarithmic Coulomb phase factors for the relative motions of the scattering aggregates,

- (ii) the asymptotically present Coulombic interactions must be subtracted from the original perturbation interactions, and

- (iii) the modified perturbation potentials (that cause the transitions of the whole system from the initial to the final states) must be short-range, square-integrable interactions (i.e. falling off faster than $1 / r$ with the augmentation of the interparticle separation $r$ ).

Overall, the correct boundary conditions (I)-(III) must be implemented by (i)-(iii) whenever there are asymptotically present Coulomb potentials in a collisional system. The reason for such a stringent requirement is in making a clear distinction between the situations before and after the collisional event. This distinction is necessary for unequivocally attributing the results of the measurements (performed always in the asymptotic region where collision has been completed) to the interaction region alone. For if there were any remaining long-range Coulomb interaction in the asymptotic scattering region, they would never cease to perturb the free channel states. This is due to the fact that Coulomb potentials produce the Coulomb logarithmic phases that do not die out even at infinitely large inter-particle distances. These Coulomb phases always distort the free channels states $\Phi_{i, f}$. Stated equivalently, when $\Phi_{i, f}$ are multiplied by the Coulomb phases, as per the request (i), the modified channel states $\Phi_{i, f}^{ \pm}$are not free any longer. Since in such situations we can no longer speak about free asymptotic states, it is useful to place the superscript \pm (symbolizing the outgoing/incoming waves) on the channel states, via $\Phi_{i, f}^{ \pm}$, to remind us that there are some Coulomb potentials (and, hence, Coulomb phases) in the asymptotic regions of the entrance and/or exit channels.

To appreciate this particular feature of Coulomb scattering theory, it is instructive to see the repercussions of obedience and disobedience of the correct boundary conditions. Thus, when the steps (I)-(III) and (i)-(iii) are accomplished in their entirety, the pertinent Møller wave operators exist and they permit the definition of the transition or $T$-operators and the scattering or $S$-matrices. In contradistinction, a disrespect of either the whole procedure (I)-(III) and (i)-(iii), or implementing these steps incompletely (e.g. in one channel, but not in the other) would mean that the Møller wave operators do not exist, with the consequence of being unable to introduce the $T$-operators and $S$-matrices [153]. Under such circumstances, attempts to deal with any particular approximate methods, in spite of the non-existent $T$-operators and $S$-matrices, are not justified. We presently illustrate how to practically implement the steps (I)-(III) and (i)-(iii) as the gestalt, regarding both the full transition amplitudes and its several approximate methods.

The purpose of this general, unified and exact setting is to lay the ground for a systematic and consistent derivation of all the existing approximate methods by making different choices of the distorting potentials and distorted wave functions. In principle, any choice of the distorting potentials and distorted wave functions is permitted as long 
as these latter two objects respect the correct boundary conditions through the steps (I)(III) and (i)-(iii). This is shown here for the four-body formulations of the continuum distorted wave (CDW-4B) [30,31], the boundary-corrected intermediate state (BCIS4B) [32], the Born distorted wave (BDW-4B) [41,42], the continuum distorted wave - initial/final state (CDW-EIS/EFS-4B) [47] and the boundary-corrected first Born (CB1-4B) [33,34] methods.

From the unified, general formalism we explicitly derive the transition amplitudes of all these methods:

- (A) with the full Coulomb wave functions (no eikonal mass limit), and

- (B) with their Coulomb phase factors (due to the eikonal mass limit).

Importantly, however, in the mentioned methods, or in any other properly designed method, the difference between the total cross sections obtained with (A) and (B) is totally negligible (barely affecting the third decimal place) from very low to very high non-relativistic impact energies.

Last but not least, the unifying formalism of the full $T$-matrices developed in the present study has an important feature in establishing the direct relationships among different distorted wave methods extracted from the general transition amplitudes. Such insights shed light onto several pertinent aspects, including these issues:

- (a) the relative performance of the analyzed distorted wave methods,

- (b) the role of the double continuum intermediate states of two electrons in the second-order methods (CDW-4B, BCIS-4B, BDW-4B, CDW-EIS/EFS-4B) relative to the first-order methods (e.g. CB1-4B) in which such effects are absent from the onset,

- (c) the extent of the influence of double electronic full Coulomb wave functions according to their inclusions in two channels, entrance and exit (CDW-4B, CDWEIS/EFS-4B) and one channel, entrance or exit (BCIS-4B, BDW-4B),

- (d) the consequences of replacing the double electronic full Coulomb wave functions by their asymptotes (CDW-EIS/EFS-4B) given by the Coulomb logarithmic phases, especially when compared to the CDW-4B method with no such additional approximations, and

- (e) the effect of using the two asymptotically equivalent logarithmic Coulomb phases for the electronic motions in one channel (CDW-EIS/EFS-4B, BDW-4B).

The conclusions can be summarized via:

- $\left(\mathrm{a}^{\prime}\right)$ At high impact energies, the CDW-4B, BCIS-4B and BDW-4B methods perform well relative to the experimental data. In sharp contrast, the CDWEIS/EFS-4B methods completely fail at all energies. On the other hand, the CB1-4B method is satisfactory at intermediate, but becomes totally inadequate at high energies.

- $\left(b^{\prime}\right)$ Twofold continuum states of the two electrons in the intermediate stage of collisions are of decisive importance in double charge exchange. It is precisely the neglect of these ionizing states that invalidates the first-order methods (CB1-4B) at high energies. In the second-order methods, large discrepancies at all energies exist among the methods that take these electronic continuum states either in a single channel (BCIS-4B, BDW-4B) or in both channels (CDW-4B, CDW-EIS/EFS-4B). 
- $\left(c^{\prime}\right)$ At lower impact energies, the double electronic continua in two channels from the CDW-4B method for double capture make the total cross sections too large. This is the same effect encountered in the CDW-3B method for single electron capture processes. However, for e.g. double capture from helium by alpha particles, at intermediate and higher energies (250-3000 keV), the total cross sections of the CDW-4B method (with or without the otherwise small contribution from the final excited heliumlike states) are too low with respect to the experimental data. For the same process, the BCIS-4B and BDW-4B methods underestimate the measurements below $1000 \mathrm{keV}$, but above this energy are in a good agreement with the experimental data at high energies.

- $\left(\mathrm{d}^{\prime}\right)$ The CDW-4B and CDW-EIS/EFS-4B methods differ in that the latter method replaces the two electronic full Coulomb waves in only one channel by their logarithmic phases. Such a replacement has a detrimental effect on the resulting total cross sections in the CDW-EIS/EFS-4B methods that underestimate the experimental data by orders of magnitudes. This completely eliminates the CDWEIS/EFS-4B methods from any useful application to double charge exchange processes.

- $\left(e^{\prime}\right)$ The only difference between the BDW-4B and CDW-EIS/EFS-4B methods is in one channel and it is in the forms of the Coulomb logarithmic phase factors. The CDW-EIS/EFS-4B methods employ the two Coulomb phases for the two electrons in terms of their distances from the same nucleus in the given channel. Both such phases have the same joint limit at infinitely large electron-nucleus distances. It is this latter Coulomb logarithmic phase factor (in terms of the asymptotic electronnucleus distances) which is used in the BDW-4B method. The ensuing total cross sections from the BDW-4B and CDW-EIS/EFS-4B methods differ by more than two orders of magnitudes at lower energies, and the discrepancy between these two theories persists to within a factor of ten at higher energies. The CDW-EIS/EFS4B methods flagrantly underestimate the experimental data that agree reasonably well with the BDW-4B method.

Overall, we can conclude that distorted wave methods for double electron capture from heliumlike atomic systems by heavy nuclei have a varying degree of success by reference to the existing measurements of total cross sections. More experiments particularly on double electron capture in collisions of fast alpha particles with helium are needed to clarify the existing controversy above $3000 \mathrm{keV}$ where the total cross sections measured by Schuch et al. [79] and Afrosimov et al. [80] differ by a factor of twenty. Among the second-order theories, the CDW-4B, BCIS-4B, BDW-4B are reasonably satisfactory, whereas the CDW-EIS/EFS-4B methods break down at all impact energies. The first-order theories (e.g. CB1-4B) are useful only at intermediate impact energies, but fail at high energies. These findings are in sharp contrast to single electron capture from heliumlike atomic systems by heavy nuclei for which the CDW-4B, BCIS-4B, BDW-4B, CDW-EIS/EFS-4B and CB1-4B methods all compare favorably to the available experimental data at intermediate and high energies. The primary reason is a significantly enhanced role of continuum intermediate states due to both electrons in double capture relative to only one active electron in single capture. In other words, compared to single capture, it follows that double capture is much 
more sensitive to the way in which the twofold ionization continua describe the intermediate stages of the collision. For single capture from heliumlike atomic systems by heavy nuclei, a simplification of the CDW-4B method through the replacement of one full electronic Coulomb waves by its asymptotic phase in one channel within the CDW-EIS/EFS-4B methods yields no significant changes in total cross sections at intermediate and high energies (below the onset of the Thomas double scattering). This ceases to be the case for double capture when two full electronic Coulomb waves from the CDW-4B method are replaced by the two asymptotic Coulomb phases in one channel in the CDW-EIS/EFS-4B methods. Such a feature might imply that e.g. triple or quadruple electron capture from multi-electron atomic targets would be even more sensitive to intermediate multiple electronic continuum states, and this would pose some new and greater challenges to the adequate theoretical prescriptions. However, before addressing such multiple capture processes from multi-electron targets, it would be important to apply the CDW-4B, BCIS-4B and BDW-4B methods to two-electron capture from multi-electron atoms $(\mathrm{C}, \mathrm{N}, \mathrm{O}, \mathrm{Ne}, \mathrm{Kr}, \mathrm{Ar})$ by heavy nuclei for which the experimental data are available at intermediate and high impact energies. Here, four-body model problems could be used with two nuclei and two active electrons, while treating the remaining target electrons only through their screening effect in the frozen core approximation.

Acknowledgements This work is supported by the research grants from Radiumhemmet at the Karolinska University Hospital and the City Council of Stockholm (FoUU) to which the author is grateful.

Open Access This article is distributed under the terms of the Creative Commons Attribution 4.0 International License (http://creativecommons.org/licenses/by/4.0/), which permits unrestricted use, distribution, and reproduction in any medium, provided you give appropriate credit to the original author(s) and the source, provide a link to the Creative Commons license, and indicate if changes were made.

\section{References}

1. Dž. Belkić, Quantum Theory of High-Energy Ion-Atom Collisions (Taylor and Francis, London, 2008)

2. Dž. Belkić, I. Mančev, J. Hanssen, Four-body methods for high-energy ion-atom collisions. Rev. Mod. Phys. 80, 249-314 (2008)

3. Dž. Belkić, Review of theories on double electron capture in fast ion-atom collisions. J. Math. Chem. 47, 1420-1467 (2010)

4. V.Yu. Lazur, M.V. Khoma, Distorted wave theories for one- and two-electron capture in fast atomic collisions. Adv. Quantum Chem. 65, 363-405 (2013)

5. V.I. Gerasimenko, L.N. Rosentsveig, Two-electron charge exchange of $\alpha$-particles in helium. J. Exp. Theor. Phys. JETP 4, 509-512 (1957) [Zh. Eksp. Teor. Fiz. 31, 684-687 (1956)]

6. V.I. Gerasimenko, The two-electron charge exchange of protons in helium during fast collisions. J. Exp. Theor. Phys. JETP 14, 789-791 (1962) [Zh. Eksp. Teor. Fiz. 41, 1104-1106 (1961)]

7. M.J. Fulton, M.H. Mittleman, Electron capture by helium ions in neutral helium. Proc. Phys. Soc. 87, 669-676 (1966)

8. K. Roy, S.C. Mukherjee, D.P. Sural, $\mathrm{H}^{-}$formation in proton-helium collisions. Phys. Rev. A 13, 987-991 (1976)

9. S. Biswas, K. Bhadra, D. Basu, Double-electron capture by protons from helium. Phys. Rev. A 15, 1900-1905 (1977)

10. C.D. Lin, Double K-shell electron capture for ion-atom collisions at intermediate energies. Phys. Rev. A 19, 1510-1516 (1979) 
11. C.D. Lin, P. Richard, Inner-shell vacancy production in ion-stom collisions. Adv. At. Mol. Phys. 17, 253-275 (1982)

12. R. Shingal, C.D. Lin, Calculations of two-electron transition cross sections between fully stripped ions and helium atoms. J. Phys. B 24, 251-264 (1991)

13. C. Chaudhuri, S. Sanyal, T.K.Rai Dastidar, Use of a diabatic molecular expansion in studies on electron capture by $\mathrm{He}^{2+}$ ions in helium at keV energies. Pramana J. Phys. 43, 175-179 (1994)

14. C. Chaudhuri, S. Sanyal, T.K.Rai Dastidar, Theoretical study of single and double charge transfer in $\mathrm{He}^{2+}-\mathrm{He}$ collisions at kilo-electron-volt energies in a diabatic molecular representation. Phys. Rev. A 52, 1137-1142 (1995)

15. C. Chaudhuri, $\mathrm{He}^{2+}-\mathrm{He}$ charge transfer collisions using a 17-state close-coupling calculations with a diabatic molecular basis. PMC Phys. B 2 (2009). https://doi.org/10.11861/1754-0429-2-2

16. T.C. Theisen, J.H. McGuire, Single and double electron capture in the independent-electron approximation at high velocities. Phys. Rev. A 20, 1406-1408 (1979)

17. R. Gayet, R.D. Rivarola, A. Salin, Double electron capture by fast nuclei. J. Phys. B 14, 2421-2427 (1981)

18. R. Gayet, Multiple capture and ionization in high-energy ion-atom collisions. J. Phys. (Paris), Colloque C1, Suppl. \# 1, 50, 53-71 (1989)

19. R. Gayet, J. Hanssen, A. Martínez, R. Rivarola, CDW and CDW-EIS investigations in an independent electron approximation for the resonant double electron capture by swift $\mathrm{He}^{2+}$ in He. Z. Phys. D 18, 345-350 (1991)

20. V.A. Sidorovich, V.S. Nikolaev, J.H. McGuire, Calculation of charge-changing cross sections in collisions of $\mathrm{H}^{+}, \mathrm{He}^{2+}$ and $\mathrm{Li}^{3+}$ with He atom. Phys. Rev. A 31, 2193-2201 (1985)

21. S.N. Chatterjee, B.N. Roy, Modified BEA calculations of $\mathrm{He}^{2+}$ impact double electron capture cross sections of atoms. J. Phys. B 18, 4283-4293 (1985)

22. M. Ghosh, C.R. Mandal, S.C. Mukherjee, Single and double electron capture from lithium by fast $\alpha$ particles. J. Phys. B 18, 3797-3803 (1985)

23. M. Ghosh, C.R. Mandal, S.C. Mukherjee, Double electron capture from helium by ions of helium, lithium, carbon and oxygen. Phys. Rev. A 35, 5259-3803 (1985)

24. R.E. Olson, A.E. Wetmore, M.L. McKenzie, Double electron transitions in collisions between multiply charged ions and helium atoms. J. Phys. B 19, L629-L634 (1986)

25. D.S.F. Crothers, R. McCarroll, Correlated continuum distorted-wave resonant double electron capture in $\mathrm{He}^{2+}-\mathrm{He}$ collisions. J. Phys. B 20, 2835-2842 (1987)

26. K.M. Dunseath, D.S.F. Crothers, Transfer and ionization processes during the collision of fast $\mathrm{H}^{+}, \mathrm{He}^{2+}$ nuclei with helium. J. Phys. B 20, 5003-5022 (1991)

27. M. Kimura, Single and double electron capture in $\mathrm{He}^{2+}+\mathrm{He}$ collisions and single electron capture in $\mathrm{He}^{+}+\mathrm{He}^{+}$collisions. J. Phys. B 21, L19-L24 (1988)

28. G. Deco, N. Grün, An approximate description of the double capture process in $\mathrm{He}^{2+}+\mathrm{He}$ collisions with static correlation. Z. Phys. D 18, 339-343 (1991)

29. M.S. Gravielle, J.E. Miraglia, Double-electron capture as a two-step process. Phys. Rev. A 45, 29652973 (1992)

30. Dž Belkić, I. Mančev, Formation of $\mathrm{H}^{-}$by double charge exchange in fast proton-helium collisions. Phys. Scr. 45, 35-42 (1992)

31. Dž Belkić, I. Mančev, Four-body CDW approximation: dependence of prior and post total cross sections for double charge exchange upon bound-state wave-functions. Phys. Scr. 46, 18-23 (1993)

32. Dž Belkić, Importance of intermediate ionization continua for double charge exchange at high energies. Phys. Rev. A 47, 3824-3844 (1993)

33. Dž Belkić, Symmetric double charge exchange in fast collisions of bare nuclei with helium-like atomic systems. Phys. Rev. A 47, 189-200 (1993)

34. Dž Belkić, Two-electron capture from helium-like atomic systems by completely stripped projectiles. J. Phys. B 26, 497-508 (1993)

35. L. Gulyás, Gy Szabo, Resonant double electron capture by fast $\mathrm{He}^{2+}$ from helium: the first-order Born approximation with correct boundary condition. Z. Phys. D 29, 115-119 (1994)

36. E. Ghanbari-Adivi, Coulomb-Born distorted wave approximation applied to the proton-helium singleelectron capture process. J. Phys. B 44, 165204 (2011)

37. E. Ghanbari-Adivi, H. Ghavaminia, Electron capture by alpha particles from helium atoms in a Coulomb-Born distorted-wave approximation. J. Phys. B 45, 235202 (2012) 
38. E. Ghanbari-Adivi, A.N. Velayati, K-shell single-electron capture in collision of fast protons with multi-electron atoms. Cent. Eur. J. Phys. 11, 423-430 (2013)

39. E. Ghanbari-Adivi, H. Ghavaminia, Double-electron capture in collision of fast alpha particles with helium atoms. Int. J. Mod. Phys. 23, 1450079 (2014)

40. H. Ghavaminia, E. Ghanbari-Adivi, Influence of electron correlations on double-capture process in proton-helium collisions. Chin. Phys. B 24, 073401 (2015)

41. Dž Belkić, Double charge exchange at high impact energies. Nucl. Inst. Methods Phys. Res. B 86, 62-81 (1994)

42. Dž Belkić, I. Mančev, M. Mudrinić, Two-electron capture from helium by fast alpha particles. Phys. Rev. A 49, 3646-3658 (1994)

43. R. Gayet, J. Hanssen, A. Martínez, R. Rivarola, Double electron capture in ion-atom collisions at high impact velocities. Nucl. Instr. Methods Phys. Res. B 86, 158-160 (1994)

44. R. Gayet, J. Hanssen, A. Martínez, R. Rivarola, Status of two-electron processes in ion-atom collisions at intermediate and high energies. Comments Atom. Mol. Phys. 30, 231-248 (1994)

45. R. Gayet, J. Hanssen, L. Jacqui, A. Martínez, R. Rivarola, Double electron capture by fast bare ions in helium atoms: production of singly and doubly excited states. Phys. Scr. 53, 549-556 (1996)

46. A.E. Martínez, H.F. Busnengo, R. Gayet, J. Hanssen, R.D. Rivarola, Double electron capture in atomic collisions at intermediate and high collision energies: contribution of capture into excited states. Nucl. Instr. Methods Phys. Res. B 132, 344-349 (1997)

47. A.E. Martínez, R.D. Rivarola, R. Gayet, J. Hanssen, Double electron capture theories: second order contributions. Phys. Scr. T80, 124-127 (1999)

48. A.L. Ford, L.A. Wehrman, K.A. Hall, J.F. Reading, Single and double electron removal from helium by protons. J. Phys. B 30, 2889-2897 (1997)

49. M. Purkait, Double electron capture cross-sections of the ground state in the collisions of $\mathrm{He}^{2+}$ and $\mathrm{Li}^{3+}$ with He. Eur. Phys. J. D 30, 11-14 (2004)

50. M. Purkait, S. Sounda, A. Dhara, C.R. Mandal, Double charge transfer cross sections in inelastic collisions of bare ions with helium atoms. Phys. Rev. A 74, 042723 (2006)

51. S. Ghosh, A. Dhara, C.R. Mandal, M. Purkait, Double electron capture cross sections from helium by fully stripped projectile ions in intermediate-to-high energies. Phys. Rev. A 78, 042708 (2008)

52. S. Ghosh, A. Dhara, C.R. Mandal, M. Purkait, Two electron capture cross sections into ground state in collision of bare projectile ions with helium atoms. Fizika A 18, 9-18 (2009)

53. S. Ghosh, A. Dhara, M. Purkait, C.R. Mandal, Double charge transfer cross sections in collisions of protons with helium. Indian J. Phys. 83, 231-243 (2010)

54. S. Samaddar, S. Halder, A. Mondal, C.R. Mandal, M. Purkait, T.K. Das, Single and double electron capture in $p-$ He and $\alpha-$ He collisions. J. Phys. B 50, 065202 (2017)

55. S.K. Alisson, Experimental results on charge-changing collisions of hydrogen and helium atoms and ions above $0.2 \mathrm{keV}$. Rev. Mod. Phys. 30, 1137-1168 (1958)

56. Ya.M. Fogel', R.V. Mitin, V.F. Kozlov, N.D. Romashko, On applicability of the Massey adiabatic hypothesis to double charge exchange. J. Exp. Theor. Phys. JETP 8, 390-398 (1959) [Zh. Eksp. Teor. Fiz. 35, 565-573 (1958)]

57. V.S. Nikolaev, L.N. Fateeva, I.S. Dmitriev, Ya.A. Teplova, Capture of several electrons by fast multicharged ions. J. Exp. Theor. Phys. JETP 14, 67-74 (1962) [Zh. Eksp. Teor. Fiz. 41, 89-99 (1961)]

58. L.I. Pivovar, M.T. Novikov, V.M. Tubaev, Electron capture by helium ions in various gases in the 300-1500 keV energy range. J. Exp. Theor. Phys. JETP 15, 1035-1039 (1962) [Zh. Eksp. Teor. Fiz. 42, 1490-1494 (1962)]

59. J.F. Williams, Cross sections for double electron capture by $2-50 \mathrm{keV}$ protons incident upon hydrogen and the inert gases. Phys. Rev. A 150, 7-10 (1966)

60. U. Schryber, Elektroneneinfang schneller protonen in gasen. Helv. Phys. Acta 40, 1023-1051 (1967)

61. K.H. Berkner, R.V. Pyle, J.W. Stearns, J.C. Warren, Single- and double-electron capture by 7.2 to 181 $\mathrm{keV}^{3} \mathrm{He}^{++}$ions in He. Phys. Rev. 166, 44-46 (1968)

62. L.H. Toburen, M.Y. Nakai, Double electron capture cross sections for incident protons in the energy range 75 to $250 \mathrm{keV}$. Phys. Rev. 177, 191-196 (1969)

63. M.B. Shah, H.B. Gilbody, Formation of $\mathrm{He}^{+}(2 s)$ metastable ions in passage of $10-60 \mathrm{keV}^{3} \mathrm{He}^{2+}$ ions through gases. J. Phys. B 7, 256-268 (1974)

64. M.B. Shah, H.B. Gilbody, Single and double ionisation of helium by $\mathrm{H}^{+}, \mathrm{He}^{2+}$ and $\mathrm{Li}^{3+}$ ions. J. Phys. B 18, 899-913 (1985) 
65. J.E. Bayfield, G.A. Khayrallah, Electron transfer in keV-energy ${ }^{4} \mathrm{He}^{++}$atomic collisions. I. Single and double electron transfer with $\mathrm{He}, \mathrm{Ar}, \mathrm{H}_{2}$ and $\mathrm{N}_{2}$. Phys. Rev. A 11, 920-929 (1975)

66. J.E. Bayfield, G.A. Khayrallah, Electron transfer in keV-energy ${ }^{4} \mathrm{He}^{++}$collisions. II. Formation of ${ }^{4} \mathrm{He}^{+{ }^{4}} \mathrm{He}^{+}(2 s)$ in collisions with $\mathrm{He}, \mathrm{Ar}, \mathrm{H}_{2}$ and $\mathrm{N}_{2}$. Phys. Rev. A 11, 930-941 (1975)

67. H. Schrey, B. Huber, Total cross section measurements for charge exchange of $\mathrm{He}^{++}$ions with $\mathrm{He}$ and Ar atoms. Z. Phys. A 273, 401-403 (1975)

68. C.W. Woods, R.L. Kauffman, K.A. Jamison, N. Stolterfoht, P. Richard, K-shell Auger-electron hypersatellites of Ne. Phys. Rev. A 12, 1393-1398 (1975)

69. E.W. McDaniel, M.R. Flannery, H.W. Ellis, F.L. Eisele, W. Pope, US Army Missile Research and Development Command, Technical Report, No. H-78-1 (1977)

70. A. Itoh, M. Asari, F. Fukuzawa, Charge-changing collisions of 0.7-2.0 MeV helium beams in various gases. I. Electron capture. J. Phys. Soc. Jpn. 48, 943-950 (1980)

71. I.S. Dmitriev, N.F. Vorob'ev, Zh.M. Konovalova, V.S. Nikolaev, V.N. Novozhilova, Ya.A. Teplova, Yu.A. Faïnberg, Loss and capture of electrons by fast ions and atoms of helium in various media. J. Exp. Theor. Phys. JETP 57, 1157-1164 (1983) [Zh. Eksp. Teor. Fiz. 84, 1987-2000 (1983)]

72. M.E. Rudd, T.V. Goffe, A. Itoh, Ionization cross sections for $10-300-\mathrm{keV} / \mathrm{u}$ and electron-capture cross sections for 5-150-keV/u ${ }^{3} \mathrm{He}^{2+}$ ions in gases. Phys. Rev. A 32, 2128-2133 (1985)

73. M. Sasao, K. Sato, A. Matsumoto, A. Nishizawa, S. Takagi, S. Amemiya, T. Masuda, Y. Tsurita, F. Fukuzawa, Y. Haruyama, Y. Kanamori, Electron capture cross sections in high energy $\mathrm{He}^{2+}+\mathrm{Li}$ collisions. J. Phys. Soc. Jpn. 55, 102-105 (1986)

74. R. Hippler, S. Datz, P. Miller, P. Pepmiler, P. Dittner, Double and single electron capture and loss in collisions of 1-2 MeV/u boron, oxygen, and silicon projectiles with helium atoms. Phys. Rev. A 35, 585-590 (1987)

75. R.D. DuBois, Ionization and charge transfer in $\mathrm{He}^{2+}$-rare gas collisions: II. Phys. Rev. A 36, 25852593 (1987)

76. N.V. de Castro Faria, F.L. Freire Jr., A.G. de Pinho, Electron loss and capture by fast helium ions in noble gases. Phys. Rev. A 37, 280-283 (1988)

77. W.K. Wu, B.A. Huber, K. Wiesemann, Cross sections for electron capture by neutral and charged particles in collisions with He. At. Data Nucl. Data Tables 42, 157-186 (1989)

78. J.H. Posthumus, P. Lukey, R. Morgenstern, Double electron capture into highly charged ions: correlated or independent? Z. Phys. D Suppl. 21, S285-S286 (1991)

79. R. Schuch, E. Justiniano, H. Vogt, G. Deco, N. Grün, Double electron capture by $\mathrm{He}^{2+}$ from He at high velocity. J. Phys. B 24, L133-L138 (1991)

80. V.V. Afrosimov, D.F. Barash, A.A. Basalaev, N.A. Guschchina, K.O. Lozhkin, V.K. Nikulin, M.N. Panov, I.Yu. Stepanov, Single- and double-electron capture from many-electron atoms by $\alpha$ particles in the MeV energy range. J. Exp. Theor. Phys. JETP 77, 554-561 (1993) [Zh. Eksp. Teor. Fiz. 104, 3297-3310 (1993)]

81. R. Dörner, V. Mergel, L. Spielberger, O. Jagutzki, H. Schmidt-Böcking, J. Ullrich, State-selective differential cross sections for double-electron capture in $0.25-0.75 \mathrm{MeV} \mathrm{He}^{2+}-\mathrm{He}$ collisions. Phys. Rev. A 57, 312-317 (1998)

82. H. Bräuning, H. Helm, J.S. Briggs, E. Salzborn, Double electron transfer in $\mathrm{H}^{-}+\mathrm{H}^{+}$collisions. J. Phys. Conf. Ser. 88, 012033 (2007)

83. M. Schulz, T. Vajnai, J.A. Brand, Differential double capture cross sections in $p-$ He collisions. Phys. Rev. A 75, 022717 (2007)

84. M.M. Sant' Anna, A.C.F. Santos, L.S.F. Coelho, G. Jalbert, N.V. de Castro Faria, F. Zappa, P. Focke, Dž. Belkić, Double electron capture cross sections for $\mathrm{Li}^{3+}$ collisions with $\mathrm{He}$ at intermediate-to-high velocities. Phys. Rev. A 80, 042707 (2009)

85. N.V. Novikov, Ya.A. Teplova, Database on charge-changing cross sections in ion-atom collisions. J. Phys. Conf. Ser. 194, 082032 (2009). http://cdfe.sinp.msu.ru/services/cccs/HTM/main.htm, http:// cdfe.sinp.msu.ru/cgibin/select.cgi?base $=$ cccs\&advanced $=$ on

86. I.S. Dmitriev, Ya.A. Teplova, Yu.A. Belkova, N.V. Novikov, Yu.A. Faïnberg, Experimental electron loss and capture cross sections in ion-atom collisions. Atom. Data Nucl. Data Tables 96, 85-121 (2010)

87. Dž. Belkić, R. Gayet, A. Salin, Electron capture in high-energy ion-atom collisions. Phys. Rep. 56, 279-369 (1979) 
88. E. Clementi, C. Roetti, Roothan-Hartree-Fock atomic wavefunctions: basis functions and their coefficients for ground and certain excited states for neutral atoms and ionized atoms, $Z \leq 54$. Atom. Data Nucl. Data Tables 14, 177-478 (1974)

89. M.R.C. McDowell, J.P. Coleman, Introduction to the Theory of Ion-Atom Collisions (North-Holland, Amsterdam, 1970)

90. P.G. Burke (ed.), Atoms in Astrophysics (Plenum, New York, 1983)

91. M. Pieksma, Electron capture cross sections at near-thermal collision energies for $\mathrm{Si}^{4+}+\mathrm{D}$. Phys. Rev. A 54, R13-R16 (1996)

92. H.H. Andersen, L.E. Rehn, Nucl. Instr. Methods Phys. Res. B 107, 1-416 (1996)

93. F. Chandezon, Critical size against Coulomb dissociation of highly charge sodium clusters obtained by ion impact. Phys. Rev. Lett. 74, 3784-3787 (1995)

94. A. Wolf (ed.), Atomic physics with stored highly charged ions, in Proceedings of "The 1st EuroConference on Atomic Physics with Stored Highly Charged Ions, Heidelberg 20-24 March 1995

95. J.P. Briand, Observation of hollow atoms or ions above insulator and metal surfaces. Phys. Rev. Lett. 77, 1452-1455 (1996)

96. The ESR experimental storage ring: cooler and mass spectrometer for highly-charged ions, in GSINachrichten 4/96 (Darmstadt, 1996)

97. K.W. Gentle, Diagnostics for magnetically confined high temperature plasmas. Rev. Mod. Phys. 67, 809-837 (1995)

98. J.C. Weisheit, Atomic excitation of intense plasmas. Adv. At. Mol. Phys. 25, 101-129 (1988)

99. M. Chabot, Stripping properties of a plasma medium for MeV/u chlorine ions. Nucl. Phys. Rev. B 51, 3504-3515 (1995)

100. M. Chabot, Stripping properties of a plasma medium for MeV/u chlorine ions. Nucl. Instrm. Methods Phys. Res. B 107, 15-18 (1996)

101. J. Botero (ed.), International Bulletin on Atomic and Molecular Data for Fusion, vol. 49 (International Atomic Energy Agency, Vienna, 1995)

102. R. Ito (ed.), Analytic cross sections for collisions of $\mathrm{H}, \mathrm{H}_{2}, \mathrm{He}$ and $\mathrm{Li}$ atoms and ions with atoms and molecules: IV. Report 96-024, Japan Atomic Energy Research Institute (1996)

103. Dž. Belkić, Review of theories on ionization in fast ion-atom collisions with prospects for applications to hadron therapy. J. Math. Chem. 47, 1366-1419 (2010)

104. Dž. Belkić (ed.), Fast Ion-Atom and Ion-Molecule Collisions. Special Issue in Book Series: Interdisciplinary Research on Particle Collisions and Quantitative Spectroscopy, vol. 1 (World Scientific Publishing, Singapore, 2013), pp. 1-310

105. Dž. Belkić (ed.), Theory of Heavy Ion Collision Physics in Hadron Therapy. Special Issue in Book Series: Advances in Quantum Chemistry, vol. 65 (2013), pp. 1-461

106. Dž. Belkić, I. Bray, A. Kadyrov, Reviews of Ion-Atom and Ion-Molecule Collisions: Theories and Experiments. Special Issue in Book Series: Interdisciplinary Research on Particle Collisions and Quantitative Spectroscopy, vol. 2 (World Scientific Publishing, Singapore, 2019), pp. 1-310 (in press)

107. R.R. Wilson, Radiological use of fast protons. Radiology 47, 487-491 (1946)

108. R.R. Wilson, Range, straggling and multiple scattering of fast protons. Phys. Rev. 71, 385-386 (1947)

109. C.A. Tobias, H.O. Anger, J.H. Lawrence, Radiological use of high energy deuterons and alpha particles. Am. J. Roentgenol. Radium Ther. Nucl. Med. 67, 1-27 (1952)

110. C.A. Tobias, J.E. Roberts, J.H. Lawrence, B.V. Low-Beer, H.O. Anger, J.L. Born, R. McCombs, C. Huggins, Irradiation hypophysectomy and related studies using $340 \mathrm{MeV}$ protons and $190 \mathrm{MeV}$ deuterons. Peaceful Uses of Atomic Energy, in Proceedings of International Conference, Geneva (1955), pp. 95-106

111. L.I. Malis, R. Loevinger, L. Kruger, J.L. Rose, Production of laminar lesions in the cerebral cortex by heavy ionizing particles. Science 126, 302-303 (1957)

112. J.H. Lawrence, C.A. Tobias, J.L. Born, R. McCombs, J.L. Roberts, H.O. Anger, B.V. Low-Beer, C. Huggins, Pituitary irradiation with high-energy proton beams: a preliminary report. Cancer Res. 18, 121-134 (1958)

113. B. Larsson, L. Leksell, B. Rexed, P. Sourander, W. Mair, B. Andersson, The high-energy proton beam as a neurosurgical tool. Nature 182, 1222-1223 (1958)

114. B. Larsson, B.A. Kihlman, Chromosome aberrations following irradiation with high-energy protons and their secondary radiation: a study of dose distributions and biological efficiency using root-tips of vicia faba and allium cepa. Int. J. Radiat. Biol. 2, 8-19 (1960) 
115. Tumor therapy with heavy ions, in GSI-Nachrichten 4/96 (Darmstadt, 1996)

116. T. Nakano, Y. Suzuki, T. Ohno, S. Kato, M. Suzuki, S. Morita, S. Sato, K. Oka, H. Tsujii, Carbon beam therapy overcomes the radiation resistance of uterine cervical cancer originating from hypoxia. Clin. Cancer Res. 12, 2185-2190 (2006)

117. D. Schulz-Ertner, O. Jäkel, W. Schlegel, Radiation therapy with charged particles. Semin. Radiat. Oncol. 16, 249-259 (2006)

118. D. Schulz-Ertner, H. Tsujii, Particle radiation therapy using proton and heavier ion beams. J. Clin. Oncol. 25, 953-964 (2007)

119. D.R. Olsen, Ø.S. Bruland, G. Frykholm, I.N. Norderhaug, Proton therapy-a systematic review of clinical effectiveness. Radiother. Oncol. 83, 123-132 (2007)

120. O. Jäkel, B. Land, S.E. Combs, D. Schulz-Ertner, J. Debus, On the cost-effectiveness of carbon ion radiation therapy for skull base chordoma. Radiother. Oncol. 83, 133-138 (2007)

121. M. Brada, M. Pijls-Johannesma, D.D. Ruysscher, Proton therapy in clinical practice: current clinical evidence. J. Clin. Oncol. 25, 965-970 (2007)

122. A. Porta, S. Agosteo, F. Campi, M. Caresana, Double-differential spectra of secondary particles from hadrons on tissue equivalent targets. Radiat. Protect. Dosim. 132, 29-41 (2008)

123. K. Kraft, S.D. Kraft, Research needed for improving heavy-ion therapy. N. J. Phys. 11, 025001 (2009)

124. D. Schardt, T. Elsässer, D. Schulz-Ertner, Heavy-ion tumor therapy: physical and radiobiological benefits. Rev. Mod. Phys. 82, 383-425 (2010)

125. E.A. Hylleraas, Neue Berechtnung der Energie des Heliums im Grundzustande, sowie tiefsten Terms von Ortho-Helium. Z. Phys. 54, 347-366 (1929)

126. P.-O. Löwdin, Studies of atomic self-consistent fields. I. Calculation of Slater functions. Phys. Rev. 90, 120-125 (1953)

127. L.C. Green, M.M. Mulder, M.N. Lewis, J.W. Woll Jr., A discussion of analytic and Hartree-Fock wave functions for $1 s^{2}$ configurations from $\mathrm{H}^{-}$to C. Phys. Rev. 93, 757-761 (1954)

128. J. Silverman, O. Platas, F.A. Matsen, Simple configuration-interaction wave functions. I. Two-electron ions: a numerical study. J. Chem. Phys. 32, 1402-1406 (1960)

129. R. Aaron, R.D. Amado, B.W. Lee, Divergence of the Green's function series for rearrangement collisions. Phys. Rev. 121, 319-323 (1961)

130. J.V. Corbett, Convergence of the Born series. J. Math. Phys. 9, 891-898 (1968)

131. K. Dettmann, G. Leibfried, Convergence behavior of the Born series for rearrangement collisions. Phys. Rev. 148, 1271-1273 (1966)

132. K.R. Greider, L.R. Dodd, Divergence of the distorted-wave Born series for rearrangement scattering. Phys. Rev. 146, 671-675 (1966)

133. L.R. Dodd, K.R. Greider, Rigorous solution of three-body scattering processes in the distorted-wave formalism. Phys. Rev. 146, 675-686 (1966)

134. L.D. Faddeev, Scattering theory for a system of three particles. J. Exp. Theor. Phys. JETP 12, 10111014 (1961) [Zh. Eksper. Teor. Fiz. 39, 1459-1467 (1960)]

135. L.D. Faddeev, S.P. Merkuriev, Quantum Scattering Theory for Several Particle Systems (Kluwer Academic Publishers, Dordrecht, 1993) [H.M. Stationary Office, Harwell, 1964]

136. L.D. Faddeev, Mathematical Problems of the Quantum Theory of Scattering for a Three-Particle System (Steklov Mathematical Institute, Leningrad, 1963) [H.M. Stationary Office, Harwell, 1964]

137. L.D. Faddeev, Mathematical Aspects of the Three-Body Problem in the Quantum Scattering Theory (Israel Program of Scientific Translations, Jerusalem, 1965)

138. I.M. Cheshire, Continuum distorted wave approximation; resonant charge transfer by fast protons in atomic hydrogen. Proc. Phys. Soc. 84, 89-98 (1964)

139. R. Gayet, Charge exchange scattering amplitude to first order of a three body expansion. J. Phys. B 5, 483-491 (1972)

140. Dž. Belkić, R. Gayet, J. Hanssen, A. Salin, The first Born approximation for charge transfer collisions. J. Phys. B 19, 2945-2953 (1986)

141. Dž. Belkić, S. Saini, H.S. Taylor, Electron capture by protons from the K-shell of H and Ar. Z. Phys. D 3, 59-76 (1986)

142. Dž. Belkić, S. Saini, H.S. Taylor, A critical test of first-order theories for electron transfer in collisions between multi-charged ions and atomic hydrogen. Phys. Rev. A 36, 1601-1617 (1987)

143. Dž. Belkić, H.S. Taylor, First-order theory for charge exchange with correct boundary conditions: general results for hydrogen-like and multi-electron targets. Phys. Rev. A 35, 1991-2006 (1987) 
144. D.P. Dewangan, J. Eichler, A first order Born approximation for charge exchange with Coulomb boundary condition. J. Phys. B 19, 2939-2944 (1986)

145. Dž. Belkić, Second Born approximation for charge exchange with correct boundary conditions. Europhys. Lett. 7, 323-327 (1988)

146. Dž. Belkić, Principles of Quantum Scattering Theory (Institute of Physics Publishing, Bristol, 2004)

147. F. Decker, J. Eichler, Exact second-order Born calculations for charge exchange with Coulomb boundary conditions. J. Phys. B 22, L95-L100 (1989)

148. F. Decker, J. Eichler, Comparative study of the distorted-wave Born and boundary-corrected Born approximation for charge exchange up to the second order. J. Phys. B 22, 3023-3036 (1989)

149. N. Toshima, A. Igarashi, Second Born approximation differential cross sections for $p+\mathrm{H}$ and $p+\mathrm{He}$ charge-exchange collisions. Phys. Rev. A 45, 6313-6317 (1992)

150. Dž. Belkić, Exact cross sections in the third Born approximation for electron capture from atomic hydrogen by protons. J. Math. Chem. (2018) (in prepaation)

151. N. Milojević, I. Mančev, Dž. Belkić, Boundary-corrected four body continuum intermediate state method for charge exchange between hydrogenlike projectiles and atoms. Phys. Rev. A 96, 032709 (2017)

152. I. Mančev, N. Milojević, Dž. Belkić, Electron capture by bare projectiles from multi-electron targets. Eur. Phys. J. D (2018) (in press)

153. J.D. Dollard, Asymptotic convergence and the Coulomb interaction. J. Math. Phys. 5, 729-738 (1964)

\section{Affiliations}

\section{Dževad Belkić ${ }^{1,2}$}

1 Department of Oncology-Pathology, Karolinska Institute, P.O. Box 260, 17176 Stockholm, Sweden

2 Medical Radiation Physics and Nuclear Medicine, Karolinska University Hospital, P.O. Box 260, 17176 Stockholm, Sweden 